\title{
Control-Relevant Modeling, Analysis, and Design for Scramjet-Powered Hypersonic Vehicles
}

\author{
Armando A. Rodriguez * Jeffrey J. Dickeson ${ }^{\dagger} \quad$ Srikanth Sridharan ${ }^{\ddagger}$ \\ Akshay Korad § Jaidev Khatri ฯ \\ Dept. of Electrical Eng., Fulton School of Eng., Arizona State University, Tempe, AZ, 85287, U.S.A. \\ Jose Benavides ॥ Don Soloway ** \\ Guidance, Navigation, and Control, NASA Ames Research Center, Moffett Field, CA, 94035, U.S.A. \\ Atul Kelkar ${ }^{\dagger \dagger}$ Jerald M. Vogel $\ddagger \ddagger$ \\ Dept. of Aerospace Eng., College of Eng., Iowa State University, Ames, IA, 50011, U.S.A.
}

\begin{abstract}
Within this paper, control-relevant vehicle design concepts are examined using a widely used 3 DOF (plus flexibility) nonlinear model for the longitudinal dynamics of a generic carrot-shaped scramjet powered hypersonic vehicle. Trade studies associated with vehicle/engine parameters are examined. The impact of parameters on control-relevant static properties (e.g. level-flight trimmable region, trim controls, AOA, thrust margin) and dynamic properties (e.g. instability and right half plane zero associated with flight path angle) are examined. Specific parameters considered include: inlet height, diffuser area ratio, lower forebody compression ramp inclination angle, engine location, center of gravity, and mass. Vehicle optimizations is also examined. Both static and dynamic considerations are addressed. The gap-metric optimized vehicle is obtained to illustrate how this controlcentric concept can be used to "reduce" scheduling requirements for the final control system. A classic inner-outer loop control architecture and methodology is used to shed light on how specific vehicle/engine design parameter selections impact control system design. In short, the work represents an important first step toward revealing fundamental tradeoffs and systematically treating control-relevant vehicle design.
\end{abstract}

\section{INTRODUCTION AND OVERVIEW}

Motivation. With the historic 2004 scramjet-powered Mach 7 and 10 flights of the X-43A ${ }^{1-4}$, hypersonics research has seen a resurgence. This is attributable to the fact that air-breathing hypersonic propulsion is viewed as the next critical step toward achieving (1) reliable, affordable, routine access to space, as well as (2) global reach vehicles. Both of these objectives have commercial as well as military implications. While rocket-based (combined cycle) propulsion systems ${ }^{5}$ are needed to reach orbital speeds, they are much more expensive to operate because they must carry oxygen. This is particularly costly when traveling at lower altitudes through the troposphere (i.e. below 36,152 ft). Current rocket-based systems also do not exhibit the desired levels of reliability and flexibility (e.g. airplane like takeoff and landing options). For this reason,

\footnotetext{
*Professor, Dept. of Electrical Engineering, Arizona State University, and AIAA Member. This research has been supported, in part, by NASA grant NNX07AC42A.

${ }^{\dagger}$ NASA PhD Fellow, Dept. of Electrical Engineering, Arizona State University, and AIAA Student Member.

${ }^{\ddagger}$ MS Student, Dept. of Electrical Engineering, Arizona State University, and AIAA Student Member.

$\S$ MS Student, Dept. of Electrical Engineering, Arizona State University, and AIAA Student Member.

『MS Student, Dept. of Electrical Engineering, Arizona State University, and AIAA Student Member.

"Hardware/Controls Engineer, Mission Critical Technologies, Inc. and AIAA Member.

**Hypersonics Project Associate Principal Investigator, NASA Ames Research Center, and AIAA Member.

${ }^{\dagger}$ Professor, Dept. of Aerospace Engineering, Iowa State University, and AIAA Member.

$\ddagger \ddagger$ Emeritus Professor, Dept. of Aerospace Engineering, Iowa State University, and AIAA Member. This research has been supported, in part, by NASA grant NNL08AA38C.
} 
much emphasis has been placed on two-stage-to-orbit (TSTO) designs that involve a turbo-ram-scramjet combined cycle first stage and a rocket-scramjet second stage. This paper focuses on control challenges associated with scramjet-powered hypersonic vehicles. Such vehicles are characterized by significant aerothermo-elastic-propulsion interactions and uncertainty ${ }^{1-18}$.

Controls-Relevant Hypersonic Vehicle Modeling. The following significant body of work (2005$2007)^{7-9,19-28}$ examines aero-thermo-elastic-propulsion modeling and control issues using a first principles nonlinear 3-DOF longitudinal dynamical model which exploits inviscid compressible oblique shock-expansion theory to determine aerodynamic forces and moments, a 1D Rayleigh flow scramjet propulsion model with a variable geometry inlet, and an Euler-Bernoulli beam based flexible model. The vehicle is $100 \mathrm{ft}$ long with weight (density) $6154 \mathrm{lb}$ per foot of depth and has a bending mode at about $21 \mathrm{rad} / \mathrm{sec}$. The controls include: elevator, stoichiometrically normalized fuel equivalency ratio (FER), diffuser area ratio (not considered in this work), and a canard (not considered in this work). A more complete description of the vehicle model can be found in previous works ${ }^{7,29}$.

More recent modeling efforts have focused on improved propulsion modeling ${ }^{30,31}$ that captures precombustion shocks, dissociation, wall heat transfer, skin friction, fuel-air mixing submodel, and finite-rate chemistry. The computational time associated with the enhanced model is significant, thus making it cumbersome for control-relevant analysis. The simple 1D Rayleigh flow engine model discussed within ${ }^{7,19,26,29}$ will be used in the current paper.

Hypersonic Vehicle Control Issues. Within this paper, we exploit the generic carrot-shaped vehicle 3DOF (plus flexibility) model presented in ${ }^{7,19,26,29}$. A myriad of issues exist that make control design for this hypersonic vehicle a potentially challenging problem:

- Input/Output Coupling. For this system, velocity control is achieved via the FER input. Flight path angle (FPA) control is achieved with the elevator ${ }^{32}$. However, there is significant coupling between $F E R$ and FPA.

- Unstable/Nonminimum Phase. Tail controlled vehicles are characterized by a non-minimum phase (right half plane, RHP) zero that is associated with the elevator to FPA map ${ }^{28}$. This RHP zero limits the achievable elevator-FPA bandidth $(B W)^{33-35}$. In addition, the rearward situated scramjet and $\mathrm{cg}$ (center of gravity), implies an inherent pitch-up vehicle instability. This instability requires a minimum $\mathrm{BW}$ for stabilization ${ }^{29}$. To address these potentially conflicting specifications, one approach has been to exploit the addition of a canard ${ }^{19,32,36-38}$. It is understood, of course, that any canard approach would face severe heating, structural, and reliability issues.

- Varying Dynamic Characteristics. Within ${ }^{29}$, it is shown that the nonlinear model changes significantly as a function of the flight condition. Specifically, it is shown that the vehicle pitch-up instability and non-minimum phase (NMP) zero vary significantly across the vehicle's trimmable region. In addition, the mass of the vehicle can be varied during a simulation in order to represent fuel consumption. Several methods have been presented in the literature to deal with the nonlinear nature of the model.

Papers addressing modeling issues include: nonlinear modeling of longitudinal dynamics ${ }^{28}$, heating effects and flexible dynamics ${ }^{9,24,39}$, FPA dynamics ${ }^{36}$, unsteady and viscous effects ${ }^{8,20}$, and high fidelity engine modeling ${ }^{30,31,40}$.

Papers addressing nonlinear control issues include: control via classic inner-outer loop architecture ${ }^{41}$, nonlinear robust/adaptive control ${ }^{32}$, robust linear output feedback ${ }^{38}$, control-oriented modeling ${ }^{19}$, linear parameter-varying control of flexible dynamics ${ }^{42}$, saturation prevention ${ }^{22,43,44}$, and thermal choking prevention $^{29,44}$.

- Uncertain Flexible Modes and Coupling to Propulsion. Flexible dynamics have been captured within the model by approximating a free-free Euler-Bernoulli beam using the assumed modes method ${ }^{24}$. Three flexible modes are used to approximate the structural dynamics. A damping factor of $\zeta=0.02$ is assumed. The associated mode frequencies are $\omega_{1}=21.02 \mathrm{rad} / \mathrm{sec}, \omega_{2}=50.87 \mathrm{rad} / \mathrm{sec}, \omega_{3}=101$ $\mathrm{rad} / \mathrm{sec}$. These modes must be adequately addressed within the control system design process. While performance can be improved by increasing controller complexity (e.g. higher order notches) ${ }^{42}$, one 
must be wary of, and careful in dealing with, modal/damping uncertainty issues. This is particularly important because structural flexing impacts the bow shock. This, in turn impacts the scramjet's inlet properties, thrust generated, aft body forces, the associated pitching moments, and hence the vehicle's attitude. Given the tight altitude-Mach flight regime - within the air-breathing corridor ${ }^{5}$ - that such vehicle must operate within, the concern is amplified. In short, one must be careful that the control system BW and complexity are properly balanced so that these lightly damped flexible modes are not overly excited.

- Control Saturation Constraints. Control saturation is of particular concern for unstable vehicles such as the one under consideration. Two specific saturation nonlinearities are a concern for any control system implementation.

- Maximum Elevator/Canard Deflection and Instability. FPA is controlled via the elevator/canard combination $^{36}$. Because these dynamics are inherently unstable, elevator saturation can result in instability ${ }^{43}$. Classical anti-windup methods may be inadequate to address the associated issues - particularly when the vehicle is open loop unstable. The constraint enforcement method within ${ }^{43,45}$ and generalized predictive control ${ }^{46}$ have been used to address such issues.

It should be noted that control surface/actuator rate limits must also be properly addressed by the control system in order to avoid instability.

- Thermal Choking/Unity FER: State Dependent Constraint. As heat is added within the combustor, the supersonic air flow is slowed. If enough heat is added, the combustor exit Mach number will approach unity, and the flow is said to be thermally choked ${ }^{47}$. If additional heat is added, the upstream conditions can be altered. This can (in principle) lead to engine unstart ${ }^{5}$ - a highly undesirable condition. The amount of FER that causes thermal choking at a particular flight condition is referred to as the thermal choking FER, or $F E R_{T C}$. In general, $F E R_{T C}$ depends upon the free-stream Mach, free-stream temperature, pressure, and density (which depend on the altitude), and the flow turn angle (vehicle geometry + AOA + elastic deflection) ${ }^{29,46}$. In addition, since the model does not capture what happens when $F E R \geq 1^{28}$, it is natural to restrict FER below unity. Given the above, it follows that the minimum of these two constraints dictates the available FER at a given flight condition. The resulting state dependent FER constraint can be computed (on-line) based on the flight condition, and must be accounted for by the control law.

Here, uncertainty is of great concern because of the potential unstart issues - issues not captured within the model. Engineers, of course, would try to "build-in protection" so that this is avoided. As such, engineers are forced to tradeoff operational envelop for enhance unstart protection.

Control-Relevant Vehicle Design Issues. Despite the successful integrated approach taken by the X43A team, as well as other prior successful flight control efforts, far too often aerospace vehicle design has not significantly involved the discipline of controls until very late in the vehicle design process or even afterwards. Research programs over the past two decades have suggested that for the anticipated hypersonic vehicles, the traditional "sequential" approach is not likely to work. This is attributable, in part, to complex uncertain nonlinear coupled unstable, non-minimum phase, flexible dynamics together with stringent flight corridor and variable constraints (e.g. specific impulse, fuel use, maximum dynamic pressure, engine temperatures and pressures). For such vehicles, an integrated multidisciplinary "parallel" approach - involving multiple disciplines up front - is essential. This is particularly true when tight flight control specifications must be satisfied in the presence of significant uncertainty.

Goals and Contributions of Paper. This paper addresses a myriad of issues that are of concern to both vehicle and control system designers. In short, this paper represents a step toward answering the following critical control-relevant vehicle design questions:

1. How do vehicle/engine design properties impact a vehicle's static and dynamic properties?

2. How do these impact control system design?

3. How should a hypersonic vehicle be designed to permit/facilitate the development of an adequately robust control system?

4. What fundamental tradeoffs exist between vehicle design objectives and vehicle control objectives? 
More specifically, in this paper we consider how the following parameters impact the static and dynamic properties of a vehicle:

- engine inlet height, diffuser area ratio, compression ramp inclination, engine location (distance behind vehicle nose), vehicle cg (center-of-gravity), and vehicle mass

Vehicle optimization is also considered. It is specifically shown that a gap-optimized vehicle can "reduce" control system scheduling requirements. A classic decentralized inner-outer loop control system architecture is used to illustrate how vehicle/engine parameter selection The gap metric represents a system-theoretic measure that quantifies the "distance" between two dynamical systems and whether or not a common controller can be deployed for the systems under consideration ${ }^{48,49}$. Within this paper, the gap metric is used to obtain a "gap-optimized vehicle" which "reduces" how much the vehicle varies throughout the trimmable region is obtained. A nonlinear pull-up maneuver is used to show that a "gap-optimized vehicle" can "reduce" control system scheduling requirements. Future work will examine the utility of pursuing gap-optimized vehicles or optimizing vehicles subject to gap constraints.

In short, this paper illustrates fundamental tradeoffs that vehicle and control system designers should jointly consider during the early stages of vehicle conceptualization/design. The paper also sheds light on how specific vehicle/engine parameter selections impact control system design - thus providing a contribution to control-relevant vehicle design. While vehicle designers may want to use a higher fidelity model (e.g. Euler based CFD with boundary layer reconstruction or Navier-Stokes based CFD ${ }^{50}$ ) to conduct more accurate vehicle trade studies, this paper shows that a (first principles) 3DOF nonlinear engineering model - such as that used in the paper - may be very useful during the early stages of vehicle conceptualization and design.

Organization of Paper. The remainder of the paper is organized as follows.

- Section II provides an overview of the dynamical model to be used in our studies.

- Section III presents engine parameter trade study results as well as a new set of nominal engine parameter values.

- Section IV presents vehicle parameter trade study results.

- Section V presents vehicle optimization results.

- Section VI discusses how control system design is impacted by vehicle/engine design parameter selection.

- Section VII summarizes the paper and presents directions for future research.

\section{DESCRIPTION OF NONLINEAR MODEL}

In this paper, we consider a first principles nonlinear 3-DOF dynamical model for the longitudinal dynamics of a generic scramjet-powered hypersonic vehicle ${ }^{7-9,19-28}$. The vehicle is $100 \mathrm{ft}$ long with weight (density) 6,154 lb per foot of depth and has a bending mode at about $22 \mathrm{rad} / \mathrm{sec}$. The controls include: elevator, stoichiometrically normalized fuel equivalency ratio (FER), diffuser area ratio (not considered in our work), and a canard. The vehicle may be visualized as shown in Figure $1^{8}$.

Modeling Approach. The following summarizes the modeling approach that has been used.

- Aerodynamics. Pressure distributions are computed using inviscid compressible oblique-shock and Prandtl-Meyer expansion theory ${ }^{10,16,28,47}$. Air is assumed to be calorically perfect; i.e. constant specific heats and specific heat ratio $\gamma \stackrel{\text { def }}{=} \frac{c_{p}}{c_{v}}=1.4^{10,47}$. A standard atmosphere is used.

Viscous drag effects (i.e. an analytical skin friction model) are captured using Eckerts temperature reference method $^{8,10}$. This relies on using the incompressible turbulent skin friction coefficient formula for a flat plate at a reference temperature. Of central importance to this method is the so-called wall temperature used. The model assumes a nominal wall temperature of $2500^{\circ} R^{8}$. While our analysis has shown that this assumption is reasonable for conducting preliminary trade studies, the wall temperature 


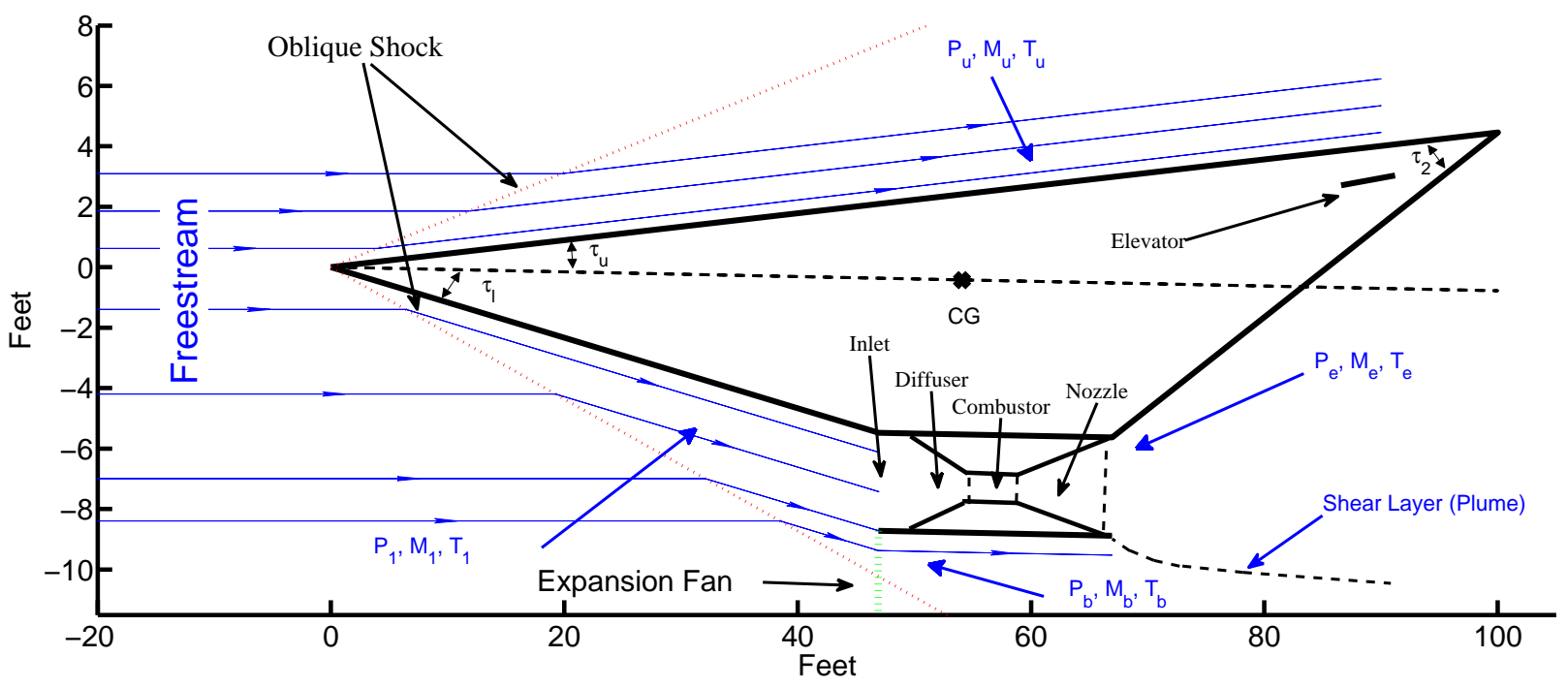

Figure 1. Schematic of Hypersonic Scramjet Vehicle

used should (in general) depend upon the flight condition being examined. As such, modeling heat transfer to the vehicle via parabolic heat equation partial differential equations (pdes) as well as modeling a suitable thermal protection system is essential for obtaining insight into wall temperature selection ${ }^{9}$. This will be addressed more comprehensively in a subsequent publication.

Unsteady effects (e.g. due to rotation and flexing) are captured using linear piston theory ${ }^{8,51}$. The idea here is that flow velocities induce pressures just as the pressure exerted by a piston on a fluid induces a velocity.

- Propulsion. A single (long) forebody compression ramp provides conditions to the rear-shifted scramjet inlet. The inlet is a variable geometry inlet (variable geometry is not exploited in our work).

The model assumes the presence of an (infinitely fast) cowl door which uses AOA to achieve shockon-lip conditions (assuming no forebody flexing). Forebody flexing, however, results in air mass flow spillage $^{28}$. At the design cruise condition, the bow shock impinges on the engine inlet (assuming no flexing). At speeds below the design-flight condition and/or larger flow turning angles, the cowl moves forward to capture the shock. At larger speeds and/or smaller flow turning angles, the bow shock is swallowed by the engine. In either case, there is a shock reflected from the cowl or within the inlet (i.e. we have a bow shock reflection). This reflected shock further slows down the flow and steers it into the engine. It should be noted that shock-shock interactions are not modeled. For example, at larger speeds and smaller flow turning angles there is a shock off of the inlet lip. This shock interacts with the bow shock. This interaction is not captured in the model.

The model uses liquid hydrogen (LH2) as the fuel. It is assumed that fuel mass flow is negligible compared to the air mass flow. The model also captures linear fuel depletion. Thrust is linearly related to FER for all expected FER values. For large FER values, the thrust levels off. In practice, when FER $>1$, the result is decreased thrust. This phenomena ${ }^{28}$ is not captured in the model. As such, control designs based on this nonlinear model (or derived linear models) should try to maintain FER below unity.

The model also captures thermal choking. In what follows, we show how to compute the FER required to induce thermal choking as well as the so-called thermal choking FER margin. The above will lead to a useful FER margin definition - one that is useful for the design of control systems for scramjet-powered hypersonic vehicles.

Finally, it should be noted that the model offers the capability for addressing linear fuel depletion. This feature was exploited for the nonlinear simulation presented in this paper.

- Structural. A single free-free Euler-Bernoulli beam partial differential equation (infinite dimensional pde) model is used to capture vehicle elasticity. As such, out-of-plane loading, torsion, and Timoshenko 
effects are neglected. The assumed modes method (based on a global basis) is used to obtain natural frequencies, mode shapes, and finite-dimensional approximants. This results in a model whereby the rigid body dynamics influence the flexible dynamics through generalized forces. This is in contrast to the model described within [28] which uses fore and aft cantilever beams (clamped at the center of gravity) and leads to the rigid body modes being inertially coupled to the flexible modes (i.e. rigid body modes directly excite flexible modes). Within the current model, forebody deflections influence the rigid body dynamics via the bow shock which influences engine inlet conditions, thrust, lift, drag, and moment ${ }^{24}$. Aftbody deflections influence the AOA seen by the elevator. As such, flexible modes influence the rigid body dynamics.

The nominal vehicle is $100 \mathrm{ft}$ long. The associated beam model is assumed to be made of titanium. It is $100 \mathrm{ft}$ long, 9.6 inches high, and $1 \mathrm{ft}$ wide (deep). This results in the nominal modal frequencies $\omega_{1}=21.02 \mathrm{rad} / \mathrm{sec}, \omega_{2}=50.87 \mathrm{rad} / \mathrm{sec}, \omega_{3}=101 \mathrm{rad} / \mathrm{sec}$. When the height is reduced to 6 inches, then we obtain the following reduced modal frequencies: $\omega_{1}=10.38 \mathrm{rad} / \mathrm{sec}, \omega_{2}=25.13 \mathrm{rad} / \mathrm{sec}$, $\omega_{3}=49.89 \mathrm{rad} / \mathrm{sec}$. Future work will examine vehicle mass-flexibility-control trade studies ${ }^{19}$.

- Actuator Dynamics. Simple first order actuator models (contained within the original model) were used in each of the control channels: elevator $-\frac{20}{s+20}$, FER $-\frac{10}{s+10}$, canard $-\frac{20}{s+20}$ (Note: canard not used in our study). These dynamics did not prove to be critical in our study. An elevator saturation of $\pm 30^{\circ}$ was used. $^{22,43}$ It should be noted, however, that these limits were never reached in our studies ${ }^{41}$. Within this paper, we consider a pull up maneuver that does not result in elevator saturation. Future work will consider more aggressive pull up maneuvers where elevator position and rate saturation become very important given the vehicle's (open loop) unstable dynamics. A (state dependent) saturation level - associated with FER (e.g. thermal choking and unity FER) - was also directly addressed ${ }^{41}$. This (velocity bandwidth limiting) nonlinearity is discussed below.

Generally speaking, the vehicle exhibits unstable non-minimum phase dynamics with nonlinear aero-elasticpropulsion coupling and critical (state dependent) FER constraints. The model contains 11 states: 5 rigid body states (speed, pitch, pitch rate, AOA, altitude) and 6 flexible states.

Unmodeled Phenomena/Effects. All models possess fundamental limitations. Realizing model limitations is crucial in order to avoid model misuse. Given this, we now provide a (somewhat lengthy) list of phenomena/effects that are not captured within the above nonlinear model. (For reference purposes, flow physics effects and modeling requirements for the X-43A are summarized within [52].)

- Dynamics. The above model does not capture longitudinal-lateral coupling and dynamics ${ }^{53}$ and the associated $6 \mathrm{DOF}$ effects.

- Aerodynamics. Aerodynamic phenomena/effects not captured in the model include the following: boundary layer growth, displacement thickness, viscous interaction, entropy and vorticity effects, laminar versus turbulent flow, flow separation, high temperature and real gas effects (e.g. caloric imperfection, electronic excitation, thermal imperfection, chemical reactions such as $0_{2}$ dissociation) ${ }^{10}$, non-standard atmosphere (e.g. troposphere, stratosphere), unsteady atmospheric effects ${ }^{6}, 3 \mathrm{D}$ effects, aerodynamic load limits.

- Propulsion. Propulsion phenomena/effects not captured in the model include the following: cowl door dynamics, multiple forebody compression ramps (e.g. three on X-43A ${ }^{54,55}$ ), forebody boundary layer transition and turbulent flow to inlet ${ }^{54,55}$, diffuser losses, shock interactions, internal shock effects, diffuser-combustor interactions, fuel injection and mixing, flame holding, engine ignition via pyrophoric silane $^{3}$ (requires finite-rate chemistry; cannot be predicted via equilibrium methods ${ }^{56}$, finite-rate chemistry and the associated thrust-AOA-Mach-FER sensitivity effects ${ }^{31}$, internal and external nozzle losses, thermal choking induced phenomena (2D and 3D) and unstart, exhaust plume characteristics, cowl door dynamics, combined cycle issues ${ }^{5}$.

Within [31], a higher fidelity propulsion model is presented which addresses internal shock effects, diffuser-combustor interaction, finite-rate chemistry and the associated thrust-AOA-Mach-FER sensitivity effects. While the nominal Rayleigh-based model (considered here) exhibits increasing thrustAOA sensitivity with increasing AOA, the more complex model in $^{31}$ exhibits reduced thrust-AOA sensitivity with increasing AOA - a behavior attributed to finite-chemistry effects. 
Future work will examine the impact of internal engine losses, high temperature gas effects, and nozzle/plume issues.

- Structures. Structural phenomena/effects not captured in the model include the following: out of plane and torsional effects, internal structural layout, unsteady thermo-elastic heating effects, aerodynamic heating due to shock impingement, distinct material properties, ${ }^{57}$ and aero-servo-elasticity ${ }^{58,59}$.

- Heating-Flexibility Issues. Finally, it should be noted that Bolender and Doman have addressed a variety of effects in their publications. For example, within $[9,24]$ the authors address the impact of heating on (longitudinal) structural mode frequencies and mode shapes.

Within [9], the authors consider a sustained two hour straight and level cruise at Mach 8, $85 \mathrm{kft}$. It is assumed that no fuel is consumed (to focus on the impact of heat addition). The paper assumes the presence of a thermal protection system (TPS) consisting of a PM2000 honeycomb outer skin followed by a layer of silicon dioxide $\left(\mathrm{SiO}_{2}\right)$ insulation. The vehicle - modeled by a titanium beam - is assumed to be insulated from the cryogenic fuel. The heat rate is computed via classic heat transfer equations that depend on speed (Mach), altitude (density), and the thermal properties of the TPS materials as well as air - convection and radiation at the air-PM2000 surface, conduction within the three TPS materials. The initial temperature of all three TPS materials was set to $559.67^{\circ} \mathrm{R}=100^{\circ} \mathrm{F}$ ). The maximum heat rate (achieved at the flight's inception) was approximately $12 \frac{B T U}{f t^{2} s e c}$ ( 1 foot aft of the nose). By the end of the two hour level flight, the average temperature within the titanium increased by $125^{\circ} \mathrm{R}$ and it was observed that the vehicle's (longitudinal) structural frequencies did not change appreciably $(<2 \%)$ [9, page 18].

When one assumes a constant $15 \frac{B T U}{f t^{2} s e c}$ heat rate at the air-PM2000 surface (same initial TPS temperature of $559.67^{\circ} R=100^{\circ} \mathrm{F}$ ), then after two hours of level flight the average temperature within the titanium increased by $200^{\circ} R$ [9, page 19]. In such a case, it can be shown that the vehicle's (longitudinal) structural frequencies do not change appreciably $(<3 \%)$. This high heat rate scenario gives one an idea by how much the flexible mode frequencies can change by. Such information is critical in order to suitably adapt/schedule the flight control system.

Comprehensive heating-mass-flexibility-control studies will be examined further in a subsequent publication.

- Actuator Dynamics. Future work will examine the impact of actuators that are rate limited; e.g. elevator, fuel pump.

It should be emphasized that the above list is only a partial list. If one needs fidelity at high Mach numbers, then many other phenomena become important; e.g. $\mathrm{O}_{2}$ dissociation ${ }^{10}$.

Longitudinal Dynamics. The equations of motion for the 3DOF flexible vehicle are given as follows:

$$
\begin{aligned}
\dot{v} & =\left[\frac{T \cos \alpha-D}{m}\right]-g \sin \gamma \\
\dot{\alpha} & =-\left[\frac{L+T \sin \alpha}{m v}\right]+q+\left[\frac{g}{v}-\frac{v}{R_{E}+h}\right] \cos \gamma \\
\dot{q} & =\frac{\mathcal{M}}{I_{y y}} \\
\dot{h} & =v \sin \gamma \\
\dot{\theta} & =q \\
\ddot{\eta} & =-2 \zeta \omega_{i} \dot{\eta}_{i}-\omega_{i}^{2} \eta_{i}+N_{i} \quad i=1,2,3 \\
\gamma & \stackrel{=}{=} \theta-\alpha \\
g & =g_{0}\left[\frac{R_{E}}{R_{E}+h}\right]^{2}
\end{aligned}
$$

where $L$ denotes lift, $T$ denotes engine thrust, $D$ denotes drag, $\mathcal{M}$ is the pitching moment, $N_{i}$ denotes generalized forces, $\zeta$ demotes flexible mode damping factor, $\omega_{i}$ denotes flexible mode undamped natural 
frequencies, $m$ denotes the vehicle's total mass, $I_{y y}$ is the pitch axis moment of inertia, $g_{0}$ is the acceleration due to gravity at sea level, and $R_{E}$ is the radius of the Earth.

- States. Vehicle states include: velocity $v, \operatorname{FPA} \gamma$, altitude $h$, pitch rate $q$, pitch angle $\theta$, and the flexible body states $\eta_{1}, \dot{\eta_{1}}, \eta_{2}, \dot{\eta_{2}}, \eta_{3}, \dot{\eta_{3}}$. These eleven (11) states are summarized in Table 1.

\begin{tabular}{|l|c|l|c|}
\hline$\sharp$ & Symbol & Description & Units \\
\hline 1 & $\mathrm{v}$ & speed & $\mathrm{kft} / \mathrm{sec}$ \\
2 & $\gamma$ & flight path angle & $\mathrm{deg}$ \\
3 & $\alpha$ & angle-of-attack (AOA) & $\mathrm{deg}$ \\
4 & $\mathrm{q}$ & pitch rate & $\mathrm{deg} / \mathrm{sec}$ \\
5 & $\mathrm{~h}$ & altitude & $\mathrm{ft}$ \\
6 & $\eta_{1}$ & $1^{\text {st }}$ flex mode & - \\
7 & $\dot{\eta_{1}}$ & $1^{\text {st }}$ flex mode rate & - \\
8 & $\eta_{2}$ & $2^{\text {nd }}$ flex mode & - \\
9 & $\dot{\eta}_{2}$ & $2^{\text {nd }}$ flex mode rate & - \\
10 & $\eta_{3}$ & $3^{\text {rd }}$ flex mode & - \\
11 & $\dot{\eta_{3}}$ & $3^{\text {rd }}$ flex mode rate & - \\
\hline
\end{tabular}

Table 1. States for Hypersonic Vehicle Model

- Controls. The vehicle has three (3) control inputs: a rearward situated elevator $\delta_{e}$, a forward situated canard $\delta_{c}{ }^{a}$, and stoichiometrically normalized fuel equivalence ratio (FER). These control inputs are summarized in Table 2. In this paper, we will only consider elevator and FER; i.e. the canard has been removed.

\begin{tabular}{|c|c|l|c|}
\hline$\sharp$ & Symbol & Description & Units \\
\hline 1 & $F E R$ & stoichiometrically normalized fuel equivalence ratio & - \\
2 & $\delta_{e}$ & elevator deflection & deg \\
3 & $\delta_{c}$ & canard deflection & deg \\
\hline
\end{tabular}

Table 2. Controls for Hypersonic Vehicle Model

In the above model, we note that the rigid body motion impacts the flexible dynamics through the generalized forces. As discussed earlier, the flexible dynamics impact the rigid body motion through thrust, lift, drag, and moment. Nominal model parameter values for the vehicle under consideration are given in Table 3 . Additional details about the model may be found within the following references ${ }^{7-9,19-28}$.

Scramjet Model. The scramjet engine model is that used in ${ }^{28,60}$. It consists of an inlet, an isentropic diffuser, a 1D Rayleigh flow combustor (frictionless duct with heat addition ${ }^{47}$ ), and an isentropic internal nozzle. A single (long) forebody compression ramp provides conditions to the rear-shifted scramjet inlet. Although the model supports a variable geometry inlet, we will not be exploiting variable geometry in this paper; i.e. diffuser area ratio $A_{d} \stackrel{\text { def }}{=} \frac{A_{2}}{A_{1}}$ will be fixed with $A_{d}=1$, see Figure 2).

Bow Shock Conditions. A bow shock will occur provided that the flow deflection angle $\delta_{s}$ is positive; i.e.

$$
\delta_{s} \stackrel{\text { def }}{=} \mathrm{AOA}+\text { forebody flexing angle }+\tau_{1 l}>0^{\circ}
$$

where $\tau_{1 l}=6.2^{\circ}$ is the lower forebody wedge angle (see Figure 1). If $\delta_{s}<0$, a Prandtl-Meyer expansion will occur. Given the above, a bow shock occurs when the following flow turning angle (FTA) condition is satisfied:

$$
\text { FTA } \stackrel{\text { def }}{=} \mathrm{AOA}+\text { forebody flexing angle }>-6.2^{\circ} .
$$

\footnotetext{
${ }^{\text {a } I n ~ t h i s ~ p a p e r, ~ w e ~ h a v e ~ r e m o v e d ~ t h e ~ c a n a r d . ~ F u t u r e ~ w o r k ~ w i l l ~ e x a m i n e ~ t h e ~ p o t e n t i a l ~ u t i l i t y ~ o f ~ a ~ c a n a r d ~ a s ~ w e l l ~ a s ~ i t s ~ v i a b i l i t y . ~}$
} 


\begin{tabular}{|c|c|c|c|}
\hline Parameter & Nominal Value & Parameter & Nominal Value \\
\hline Total Length (L) & $100 \mathrm{ft}$ & Lower forebody angle $\left(\tau_{1 L}\right)$ & $6.2^{\circ}$ \\
\hline Forebody Length $\left(\mathrm{L}_{1}\right)$ & $47 \mathrm{ft}$ & Tail angle $\left(\tau_{2}\right)$ & $14.342^{\circ}$ \\
\hline Aftbody Length $\left(\mathrm{L}_{2}\right)$ & $33 \mathrm{ft}$ & Mass per unit width & 191.3024 slugs $/ \mathrm{ft}$ \\
\hline Engine Length & $20 \mathrm{ft}$ & Weight per unit width & $6,154.1 \mathrm{lbs} / \mathrm{ft}$ \\
\hline Engine inlet height $\mathrm{h}_{i}$ & $3.25 \mathrm{ft}$ & Mean Elasticity Modulus & $8.6482 \times 10^{7} \mathrm{psi}$ \\
\hline Upper forebody angle $\left(\tau_{1 U}\right)$ & $3^{o}$ & Moment of Inertia $\mathrm{I}_{y y}$ & 86,723 slugs $\mathrm{ft}^{2} / \mathrm{ft}$ \\
\hline Elevator position & $(-85,-3.5) \mathrm{ft}$ & Center of gravity & $(-55,0) \mathrm{ft}$ \\
\hline Diffuser exit/inlet area ratio & 1 & Elevator Area & $17 \mathrm{ft}^{2}$ \\
\hline Titanium Thickness & 9.6 in & Nozzle exit/inlet area ratio & 6.35 \\
\hline First Flex. Mode $\left(\omega_{n_{1}}\right)$ & $22.2 \mathrm{rad} / \mathrm{s}$ & Second Flex. Mode $\left(\omega_{n_{2}}\right)$ & $48.1 \mathrm{rad} / \mathrm{s}$ \\
\hline Third Flex. Mode $\left(\omega_{n_{3}}\right)$ & $94.8 \mathrm{rad} / \mathrm{s}$ & Flex. Mode Damping $(\zeta)$ & 0.02 \\
\hline
\end{tabular}

Table 3. Vehicle Nominal Parameter Values

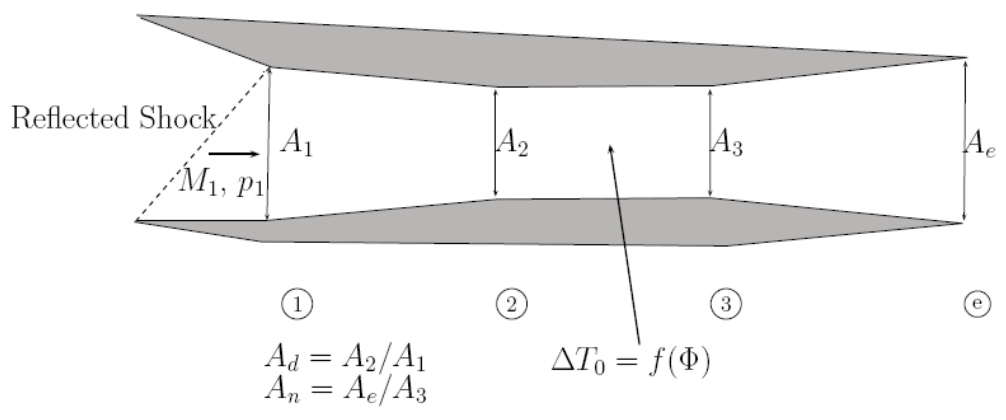

Figure 2. Schematic of Scramjet Engine

Properties Across Bow Shock. Let $\left(M_{\infty}, T_{\infty}, p_{\infty}\right)$ denote the free-stream Mach, temperature, and pressure. Let $\gamma \stackrel{\text { def }}{=} \frac{c_{p}}{c_{v}}=1.4$ denote the specific heat ratio for air - assumed constant in the model; i.e. air is calorically perfect. ${ }^{10}$ The shock wave angle $\theta_{s}=\theta_{s}\left(M_{\infty}, \delta_{s}, \gamma\right)$ can be found as the middle root (weak shock solution) of the following shock angle polynomial ${ }^{28,47}$ :

$$
\sin ^{6} \theta_{s}+b \sin ^{4} \theta_{s}+\operatorname{csin}^{2} \theta_{s}+d=0
$$

where

$$
b=-\frac{M_{\infty}^{2}+2}{M_{\infty}^{2}}-\gamma \sin ^{2} \delta_{s} \quad c=\frac{2 M_{\infty}^{2}+1}{M_{\infty}^{4}}+\left[\frac{(\gamma+1)^{2}}{4}+\frac{\gamma-1}{M_{\infty}^{2}}\right] \sin ^{2} \delta_{s} \quad d=-\frac{\cos ^{2} \delta_{s}}{M_{\infty}^{4}}
$$

The above can be addressed by solving the associated cubic in $\sin ^{2} \theta_{s}$. A direct solution is possible if Emanuel's 2001 method is used [47, page 143].

After determining the shock wave angle $\theta_{s}$, one can determine properties across the bow shock using classic relations from compressible flow [47, page 135]; i.e. $M_{s}, T_{s}, p_{s}$ - functions of $\left(M_{\infty}, \delta_{s}, \gamma\right)$ :

$$
\begin{aligned}
\frac{T_{s}}{T_{\infty}} & =\frac{\left(2 \gamma M_{\infty}^{2} \sin ^{2} \theta_{s}+1-\gamma\right)\left((\gamma-1) M_{\infty}^{2} \sin ^{2} \theta_{s}+2\right)}{(\gamma+1)^{2} M_{\infty}^{2} \sin ^{2} \theta_{s}} \\
\frac{p_{s}}{p_{\infty}} & =1+\frac{2 \gamma}{\gamma+1}\left(M_{\infty}^{2} \sin ^{2} \theta_{s}-1\right) \\
M_{s}^{2} \sin ^{2}\left(\theta_{s}-\delta_{s}\right) & =\frac{M_{\infty}^{2} \sin ^{2} \theta_{s}(\gamma-1)+2}{2 \gamma M_{\infty}^{2} \sin ^{2} \theta_{s}-(\gamma-1)}
\end{aligned}
$$


It should be noted that for large $M_{\infty}$, the computed temperature $T_{s}$ across the shock will be larger than it should be because our assumption that air is calorically perfect (i.e. constant specific heats) does not capture other forms of energy absorption; e.g. electronic excitation and chemical reactions ${ }^{10}$.

Properties Across Prandtl-Meyer Expansion. An expansion fan occurs when there is a flow over a convex corner; i.e. flow turns away from itself. More specifically to the bow, if $\delta_{s}<0$ a Prandtl-Meyer expansion will occur. To determine the properties across the expansion, let $\left(M_{\infty}, T_{\infty}, p_{\infty}\right)$ denote the free-stream (supersonic) Mach, temperature, and pressure, respectively. If we let $\delta=-\delta_{s}>0$ denote the expansion ramp angle (in radians), the properties across the expansion fan $\left(M_{e}, T_{e}, p_{e}\right)$ can be calculated as follows ${ }^{28,47}$ :

$$
\begin{aligned}
\nu_{1} & =\sqrt{\frac{\gamma+1}{\gamma-1}} \tan ^{-1}\left(\sqrt{\frac{\gamma-1}{\gamma+1}\left(M_{\infty}^{2}-1\right)}\right)-\tan ^{-1}\left(\sqrt{M_{\infty}^{2}-1}\right) \\
\nu_{2} & =\nu_{1}+\delta \\
f\left(M_{e}\right) & =\sqrt{\frac{\gamma+1}{\gamma-1}} \tan ^{-1}\left(\sqrt{\frac{\gamma-1}{\gamma+1}\left(M_{e}^{2}-1\right)}\right)-\tan ^{-1}\left(\sqrt{M_{e}^{2}-1}\right)-\nu_{2}=0 \\
\frac{P_{e}}{P_{\infty}} & =\left[\frac{1+\frac{\gamma-1}{2} M_{\infty}^{2}}{1+\frac{\gamma-1}{2} M_{e}^{2}}\right] \\
\frac{T_{e}}{T_{\infty}} & =\left[\frac{1+\frac{\gamma-1}{2} M_{\infty}^{2}}{1+\frac{\gamma-1}{2} M_{e}^{2}}\right]
\end{aligned}
$$

$\nu_{1}$ is the angle for which a Mach 1 flow must be expanded to attain the free stream Mach.

Translating Cowl Door. The model assumes the presence of an (infinitely fast) translating cowl door which uses AOA to achieve shock-on-lip conditions (assuming no forebody flexing). Forebody flexing, however, results in an oscillatory bow shock and air mass flow spillage ${ }^{28}$. A bow shock reflection (off of the cowl or inside the inlet) further slows down the flow and steers it into the engine. Shock-shock interactions are not modeled.

- Impact of Having No Cowl Door. Associated with a translating cowl door are potentially very severe heating issues. For our vehicle, the translating cowl door can extend a great deal. For example, at Mach 5.5, 70kft, the trim FTA is $1.8^{\circ}$ and the cowl door extends $14.1 \mathrm{ft}$. Of particular concern, due to practical cowl door heating/structural issues, is what happens when the cowl door is over extended through the bow shock. This occurs, for example, when structural flexing results in a smaller FTA (and hence a smaller bow shock angle) than assumed by the rigid-body shock-on-lip cowl door extension calculation. This is certainly a major concern. It leads one to ask the question: What happens to the vehicle properties if no cowl door is present? When the FTA is large or when the vehicle Mach is low, the shock angle increases and more air mass spillage would occur. Our analysis shows that the impact of neglecting the cowl door on the vehicle's static properties is significant while the impact on the vehicle's dynamic properties is negligible. This will receive further examination in a subsequent publication.

Inlet Properties. The bow reflection turns the flow parallel into the scramjet engine ${ }^{28}$. The oblique shock relations are implemented again, using $M_{s}$ as the free-stream input, $\delta_{1}=\tau_{1 l}$ as the flow deflection angle to obtain the shock angle $\theta_{1}=\theta_{1}\left(M_{s}, \delta_{1}, \gamma\right)$ and the inlet (or diffuser entrance) properties: $M_{1}, T_{1}, p_{1}$ functions of $\left(M_{s}, \theta_{1}, \gamma\right)$.

Diffuser Exit-Combustor Entrance Properties. The diffuser is assumed to be isentropic. The combustor entrance properties are therefore found using the formulae in ${ }^{28}$, [47, pp. 103-104] $-M_{2}=M_{2}\left(M_{1}, A_{d}, \gamma\right)$, 


$$
\begin{aligned}
T_{2}=T_{2}\left(M_{1}, M_{2}, \gamma\right), p_{2}=p_{2}\left(M_{1}, M_{2}, \gamma\right): & \\
\frac{\left[1+\frac{\gamma-1}{2} M_{2}^{2}\right]^{\frac{\gamma+1}{\gamma-1}}}{M_{2}^{2}} & =A_{d}^{2} \frac{\left[1+\frac{\gamma-1}{2} M_{1}^{2}\right]^{\frac{\gamma+1}{\gamma-1}}}{M_{1}^{2}} \\
T_{2} & =T_{1}\left[\frac{1+\frac{1}{2}(\gamma-1) M_{1}^{2}}{1+\frac{1}{2}(\gamma-1) M_{2}^{2}}\right] \\
p_{2} & =p_{1}\left[\frac{1+\frac{1}{2}(\gamma-1) M_{1}^{2}}{1+\frac{1}{2}(\gamma-1) M_{2}^{2}}\right]^{\frac{\gamma}{\gamma-1}}
\end{aligned}
$$

where $A_{d} \stackrel{\text { def }}{=} \frac{A_{2}}{A_{1}}$ is the diffuser area ratio. Also, one can determine the total temperature $T_{t_{2}}=T_{t_{2}}\left(T_{2}, M_{2}, \gamma\right)$ at the combustor entrance can be found using [47, page 80]:

$$
T_{t 2}=\left[1+\frac{\gamma-1}{2} M_{2}^{2}\right] T_{2} .
$$

Since $A_{d}=1$ in the model, it follows that $M_{2}=M_{1}, T_{2}=T_{1}, p_{2}=p_{1}$, and $T_{t 2}=\left[1+\frac{\gamma-1}{2} M_{1}^{2}\right] T_{1}=T_{t_{1}}$.

FER. The model uses liquid hydrogen (LH2) as the fuel. If $f$ denotes fuel-to-air ratio and $f_{s t}$ denotes stoichiometric fuel-to-air ratio, then the stoichiometrically normalized fuel equivalency ratio is given by $F E R \stackrel{\text { def }}{=} \frac{f}{f_{s t}},{ }^{5}{ }^{28} \mathrm{FER}$ is the engine control. While FER is primarily associated with the vehicle velocity, its impact on FPA is significant (since engine is situated below vehicle $\mathrm{cg}$ ). This coupling will receive further examination in what follows.

Combustor Exit Properties. In this model, we have a constant area combustor where the combustion process is captured via heat addition. To determine the combustor exit properties, one first determines the change in total temperature across the combustor ${ }^{28}$ :

$$
\Delta T_{c}=\Delta T_{c}\left(T_{t_{2}}, F E R, H_{f}, \eta_{c}, c_{p}, f_{s t}\right)=\left[\frac{f_{s t} F E R}{1+f_{s t} F E R}\right]\left(\frac{H_{f} \eta_{c}}{c_{p}}-T_{t_{2}}\right)
$$

where $H_{f}=51,500 \mathrm{BTU} / \mathrm{lbm}$ is the heat of reaction for liquid hydrogen (LH2), $\eta_{c}=0.9$ is the combustion efficiency, $c_{p}=0.24 \mathrm{BTU} / \mathrm{lbm}^{\circ} \mathrm{R}$ is the specific heat of air at constant pressure, and $f_{s t}=0.0291$ is the stoichiometric fuel-to-air ratio for $\mathrm{LH} 2^{5}$. Given the above, the Mach $M_{3}$, temperature $T_{3}$, and pressure $p_{3}$ at the combustor exit are determined by the following classic 1D Rayleigh flow relationships ${ }^{28}$, [47, pp. 103-104]:

$$
\begin{aligned}
\frac{M_{3}^{2}\left[1+\frac{1}{2}(\gamma-1) M_{3}^{2}\right]}{\left(\gamma M_{3}^{2}+1\right)^{2}} & =\frac{M_{2}^{2}\left[1+\frac{1}{2}(\gamma-1) M_{2}^{2}\right]}{\left(\gamma M_{2}^{2}+1\right)^{2}}+\left[\frac{M_{2}^{2}}{\left(\gamma M_{2}^{2}+1\right)^{2}}\right] \frac{\Delta T_{c}}{T_{2}} \\
T_{3} & =T_{2}\left[\frac{1+\gamma M_{2}^{2}}{1+\gamma M_{3}^{2}}\right]^{2}\left(\frac{M_{3}}{M_{2}}\right)^{2} \\
p_{3} & =p_{2}\left[\frac{1+\gamma M_{2}^{2}}{1+\gamma M_{3}^{2}}\right] .
\end{aligned}
$$

Given the above, one can then try to solve equation (26) for $M_{3}=M_{3}\left(M_{2}, \frac{\Delta T_{c}}{T_{2}}, \gamma\right)$. This will have a solution provided that $M_{2}$ is not too small, $\Delta T_{c}$ is not too large (i.e. $F E R$ is not too large or $T_{2}$ is not too small. See discussion below.

Thermal Choking FER $\left(M_{3}=1\right)$. Once the change in total temperature $\Delta T_{c}=\Delta T_{c}\left(T_{t_{2}}, F E R, H_{f}, \eta_{c}, c_{p}, f_{s t}\right)$ across the combustor has been computed, it can be substituted into equation (26) and one can "try" to solve for $M_{3}$. Since the left hand side of equation (26) lies between 0 (for $M_{3}=0$ ) and 0.2083 (for $M_{3}=1$ ), it follows that if the right hand side of equation (26) is above 0.2083 then no solution for $M_{3}$ exists. Since the first term on the right hand side of equation (26) also lies between 0 and 0.2083 , it follows that this occurs when $\Delta T_{c}$ is too large; i.e. too much heat is added into the combustor or too high an FER. In short, a solution $M_{3}$ will exist provided that FER is not too large, $T_{2}$ is not too small (i.e. altitude not too high), and the combustor entrance Mach $M_{2}$ is not too small (i.e. FTA not too large). When $M_{3}=1$, a condition referred to as thermal choking ${ }^{5,47}$ is said to exist. The FER that produces this we call the thermal choking $F E R$ - denoted $F E R_{T C}$. In general, $F E R_{T C}$ will be a function of the following: $M_{\infty}, T_{\infty}$, and FTA. 
Physically, the addition of heat to a supersonic flow causes it to slow down. If the thermal choking FER $\left(F E R_{T C}\right)$ is applied, then we will have $M_{3}=1$ (i.e. sonic combustor exit). When thermal choking occurs, it is not possible to increase the air mass flow through the engine. Propulsion engineers want to operate near thermal choking for engine efficiency reasons ${ }^{5}$. However, if additional heat is added, the upstream conditions can be altered and it is possible that this may lead to engine unstart. This is highly undesirable. For this reason, operating near thermal choking has been described by some propulsion engineers as "operating near the edge of a cliff." In general, thermal choking will occur if FER is too high, $M_{\infty}$ is too low, altitude is too high $\left(T_{\infty}\right.$ too low), FTA is too high. See discussion below.

Internal Nozzle. The exit properties $M_{e}=M_{e}\left(M_{3}, A_{n}, \gamma\right), T_{e}=T_{e}\left(M_{3}, M_{e}, \gamma\right), p_{e}=p_{e}\left(M_{3}, M_{e}, \gamma\right)$ of the scramjet's isentropic internal nozzle are founds as follows:

$$
\begin{aligned}
\frac{\left[1+\frac{\gamma-1}{2} M_{e}^{2}\right]^{\frac{\gamma+1}{\gamma-1}}}{M_{e}^{2}} & =A_{n}^{2} \frac{\left[1+\frac{\gamma-1}{2} M_{3}^{2}\right]^{\frac{\gamma+1}{\gamma-1}}}{M_{3}^{2}} \\
T_{e} & =T_{3}\left[\frac{1+\frac{1}{2}(\gamma-1) M_{3}^{2}}{1+\frac{1}{2}(\gamma-1) M_{e}^{2}}\right] \\
p_{e} & =p_{3}\left[\frac{1+\frac{1}{2}(\gamma-1) M_{3}^{2}}{1+\frac{1}{2}(\gamma-1) M_{e}^{2}}\right]^{\frac{\gamma}{\gamma-1}}
\end{aligned}
$$

where $A_{n} \stackrel{\text { def }}{=} \frac{A_{e}}{A_{3}}$ is the internal nozzle area ratio (see Figure 2). $A_{n}=6.35$ is used in the model.

Thrust due to Internal Nozzle. The purpose of the expanding internal nozzle is to recover most of the potential energy associated with the compressed (high pressure) supersonic flow. The thrust produced by the scramjet's internal nozzle is given by ${ }^{47}$

$$
\text { Thrust }_{\text {internal }}=\dot{m}_{a}\left(v_{e}-v_{\infty}\right)+\left(p_{e}-p_{\infty}\right) A_{e}
$$

where $\dot{m}_{a}$ is the air mass flow through the engine, $v_{e}$ is the exit flow velocity, $v_{\infty}$ is the free-stream flow velocity. $p_{e}$ is the pressure at the engine exit plane, $A_{1}$ is the engine inlet area, $A_{e}$ is the engine exit area, $v_{e}=M_{e} \operatorname{sos}_{e}, v_{\infty}=M_{\infty} \operatorname{sos}_{\infty}, \operatorname{sos}_{e}=\sqrt{\gamma R T_{e}}, \operatorname{sos}_{\infty}=\sqrt{\gamma R T_{\infty}}$, and $R$ is the gas constant for air. Because we assume that the internal nozzle to be symmetric, this internal thrust is always directed along the vehicle's body axis. The mass air flow into the inlet is given as follows:

$$
\dot{m}_{a}= \begin{cases}p_{\infty} M_{\infty} \sqrt{\frac{\gamma}{R T_{\infty}}}\left[L_{1} \frac{\sin \left(\tau_{1 l}-\alpha\right)}{\tan \left(\tau_{1 l}\right)}+h_{i} \cos (\alpha)\right] & \text { Oblique bow shock (swallowed by engine) } \\ p_{\infty} M_{\infty} \sqrt{\frac{\gamma}{R T_{\infty}}} h_{i}\left[\frac{\sin \left(\theta_{s}\right) \cos \left(\tau_{11}\right)}{\sin \left(\theta_{s}-\alpha-\tau_{1 l}\right)}\right] & \text { Oblique bow shock - shock on lip } \\ p_{\infty} M_{\infty} \sqrt{\frac{\gamma}{R T_{\infty}}} h_{i} \cos \left(\tau_{1 l}\right) & \text { Lower forebody expansion fan }\end{cases}
$$

External Nozzle. The purpose of the expanding external nozzle is recover the rest of the potential energy associated with the compressed supersonic flow. A nozzle that is too short would not be long enough to recover the stored potential energy. In such a case, the nozzle's exit pressure would be larger than the free stream pressure and we say that it is under-expanded [47, Page-209]. The result is reduced thrust. A nozzle that is too long would result in the nozzle's exit pressure being smaller than the free stream pressure and we say that it is over-expanded [47, Page-209]. The result, again, is reduced thrust. When the nozzle length is "properly selected," the exit pressure is equal to the free stream pressure and maximum thrust is produced. Within [61, page 5], ${ }^{62}$ the authors say that the optimum nozzle length is about 7 throat heights. This includes the internal as well as the external nozzle. For our vehicle, the internal nozzle has no assigned length. This becomes an issue when internal losses are addressed. For the Bolender, et. al. model, the external nozzle length is 10.15 throat heights (with throat height $h_{i}=3.25 \mathrm{ft}$ ). For the new engine design presented later on in this paper, the external nozzle length is 7.33 throat heights (with throat height $h_{i}=4.5 \mathrm{ft}$ ). The external nozzle contributes a force on the upper aft body. This force can be resolved into 2 components - the component along the fuselage water line is said to contribute to the total thrust. This component is given by the expression:

$$
\text { Thrust }_{\text {external }}=p_{\infty} L_{a}\left(\frac{p_{e}}{p_{\infty}}\right)\left[\frac{\ln \left(\frac{p_{e}}{p_{\infty}}\right)}{\frac{p_{e}}{p_{\infty}}-1}\right] \tan \left(\tau_{2}+\tau_{1 U}\right)
$$


Plume Assumption. The engine's exhaust is bounded above by the aft body/nozzle and below by the shear layer between the gas and the free stream atmosphere. The two boundaries define the shape of the external nozzle. Within [60, page 1315], ${ }^{7}$, a critical assumption is made regarding the shape of the external nozzle-and-plume in order to facilitate (i.e. speed up) the calculation of the aft body pressure distribution. In short, the so-called "plume assumption" implies that the external nozzle-and-plume shape does not change with respect to the vehicle's body axes. This implies that the plume shape is independent of the flight condition. Our (limited) studies to date show that this assumption is suitable for preliminary trade studies but a higher fidelity aft body pressure distribution calculation is needed to understand how properties change over the trimmable region. In short, our fairly limited studies suggest that the plume assumption impacts static properties significantly while dynamic properties are only mildly impacted. The impact of the plume assumption will be examined further in a subsequent publication.

Total Thrust. The total thrust is obtained by adding the thrust due to the internal and external nozzles.

Trimmable Region and Vehicle Properties. Within this paper (and all our work to date), trim refers to a non-accelerating state; i.e. no translational or rotational acceleration. Moreover, all trim analysis has focused on level flight. Figure 3 shows the level-flight trimmable region for the nominal vehicle being considered $^{7,19,26,29,41}$ (using the original nominal engine parameters). We are interested in how the static and dynamic properties of the vehicle vary across this region. Static properties of interest include: trim controls (FER and elevator), internal engine variables (e.g. temperature and pressure), thrust, thrust margin, AOA, L/D. Dynamic properties of interest include: vehicle instability and RHP transmission zero associated with FPA. Understanding how these properties vary over the trimmable region is critical for designing a robust nonlinear (gain-schedulted/adaptive) control system that will enable flexible operation. For example, consider a TSTO flight. The mated vehicles might fly up along $\bar{q}=2000$ psf to a desired altitude, then conduct a pull-up maneuver to reach a suitable staging altitude.

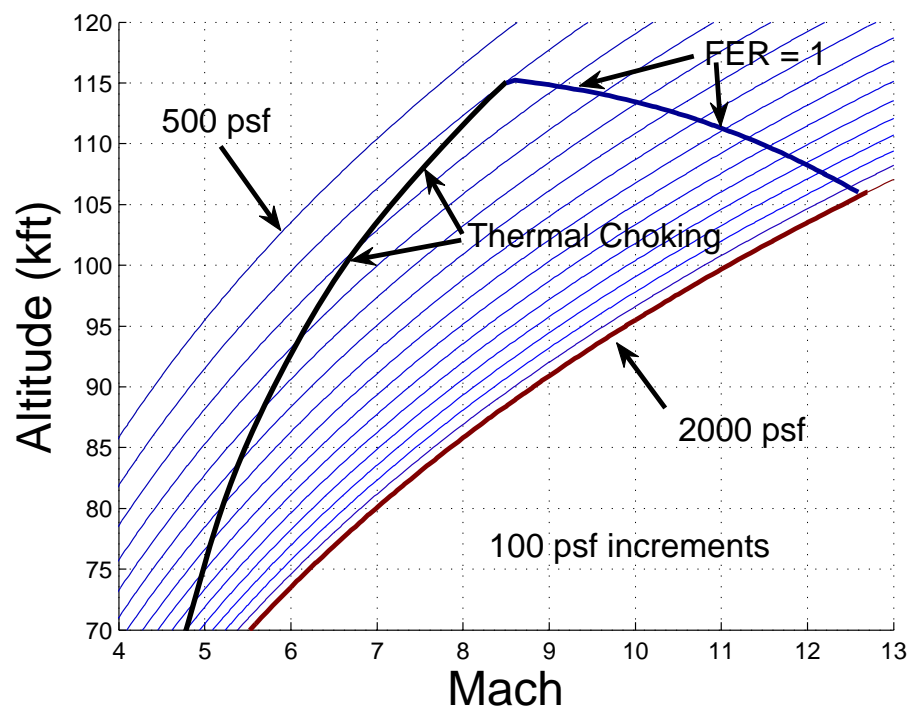

Figure 3. Visualization of Trimmable Region: Level-Flight, Unsteady-Viscous Flow, Flexible Vehicle, 2 Controls

\section{Engine Parameter Studies}

This section examines the impact of varying the engine inlet height $h_{i}$ and the diffuser area ratio $A_{d}$. Three basic engine designs were considered: (1) current (nominal, slow or small), (2) new (intermediate speed or size), and (3) aggressive (fast or large). In what follows, $h_{e}$ denotes the internal nozzle exit height and $A_{n}$ is the internal nozzle area ratio. 
1. Current (Nominal, Slow or Small) Engine Design. The current (nominal, slow or small) engine design parameters are as follows ${ }^{7}$ :

$$
\text { - } h_{i}=3.25 \quad h_{e}=5 \quad A_{d}=1 \quad A_{n}=6.35 .
$$

These parameters are not geometrically compatible with the vehicle shown in Figures 1 and 2; i.e. it would be impossible for the vehicle to have the pictorially implied flat base; i.e. internal nozzle exit height $h_{e}$ equal to inlet height $h_{i}$.

Given the above, we set out to examine engines with $h_{e}=h_{i}$. This implies that $A_{n}=\frac{1}{A_{d}}$.

2. New (Intermediate Speed or Size) Engine Design. The new (intermediate speed or size) engine design parameters were selected as follows:

$$
\text { - } h_{e}=h_{i}=4.5 \quad A_{d}=0.15 \quad A_{n}=\frac{1}{A_{d}}=6.67 .
$$

It should be noted that the value $A_{d}=0.1$ was used within ${ }^{30,31}$. This new engine design will be used later in the paper for analysis and control system design purposes.

3. Aggressive (Fast or Large) Engine Design. An aggressive (fast or large) engine design was also considered:

$$
\text { - } h_{e}=h_{i}=6 \quad A_{d}=0.125 \quad A_{n}=\frac{1}{A_{d}}=8 .
$$

Constraints for Engine Parameter Trade Studies (Mach 8, 85 kft, Level Flight). The above engines were obtained by conducting parametric trade studies at Mach 8, $85 \mathrm{kft}$, level flight. The following constraints were assumed in our studies:

- Flat base (internal nozzle exhaust height $h_{e}$ equal to inlet height $h_{i}$ ); i.e. $h_{e}=h_{i}$ and $A_{n}=A_{d}^{-1}$;

- Inlet height $h_{i}$ was varied between $\pm 50 \%$ of nominal $3.25 \mathrm{ft}$;

- Engine mass $m_{\text {engine }}$ was varied between $\pm 50 \%$ of nominal $10 \mathrm{klbs}$;

- Diffuser area ratio $A_{d}$ was varied between 0.1 and 0.35 .

\section{III..1. Impact of Engine Parameters on Static Properties (Mach 8, 85 kft, Level Flight)}

Figure 4 shows the impact of varying $\left(h_{i}, A_{d}\right)$ on FER, combustor temperature (assuming calorically perfect air), thrust, thrust margin at Mach 8, $85 \mathrm{kft}$, level flight.

Trim FER. From Figure 4 (upper left), one observes that the:

- trim FER decreases with decreasing $A_{d}$ for a fixed $h_{i}$;

- trim FER decreases with increasing $h_{i}$ when $h_{i}<7$.

These suggests choosing $A_{d}$ small (i.e. significant diffuser compression) and $h_{i}$ large (i.e. large air mass flow) in order to achieve a small trim FER. The above, however, does not tell the full story since fuel consumption (trim fuel rate) - shown in Figure 4 (upper right) - increases with increasing $h_{i}$, and the thrust margin decreases for $A_{d}<0.125$.

Trim Combustor Temperature. From Figure 4 (lower left), one also observes that:

- Trim combustor temperature is a concave up function of $\left(h_{i}, A_{d}\right)$ - minimized at $h_{i} \approx 5.5, A_{d} \approx 0.125$.

- Trim combustor temperature exhibits a steep gradient for $A_{d}>0.2$ 
FER at Mach $8,85 \mathrm{kft}$
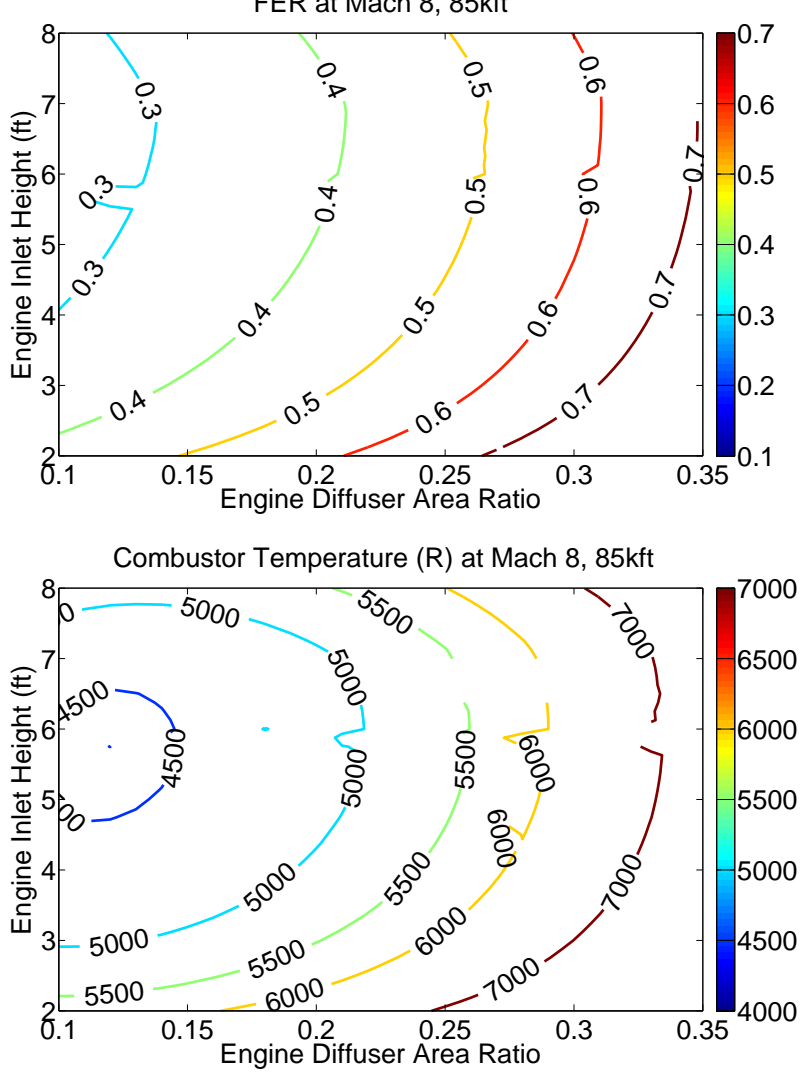

Fuel consumption (slugs/s) at Mach 8, 85kft

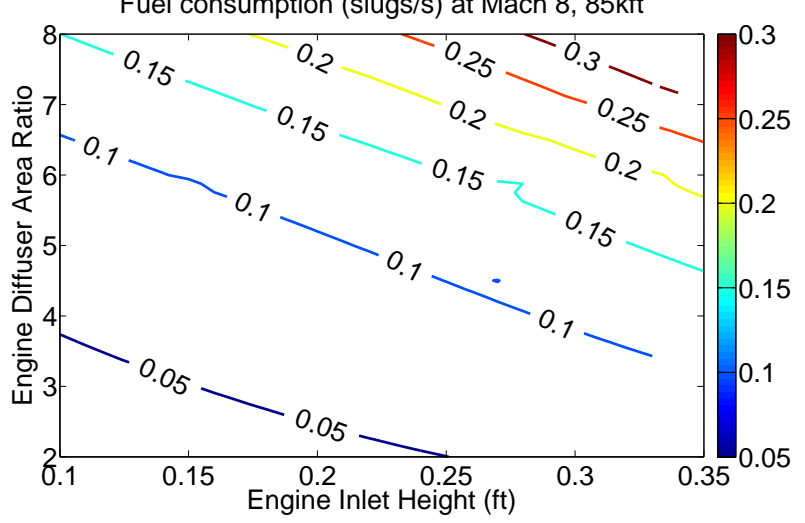

Thrust Margin at Mach 8, 85kft

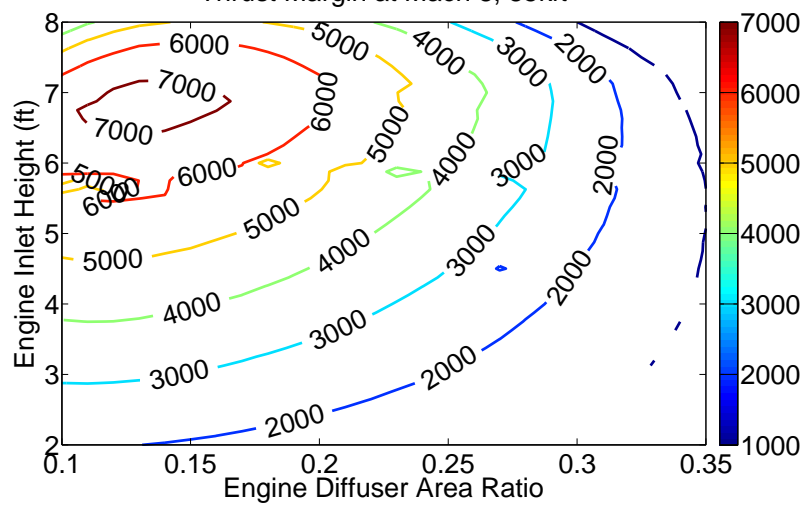

Figure 4. Trim FER, Combustor Temperature, Thrust, Thrust Margin: Dependence on $h_{i}, A_{d}$ (Mach 8, 85 kft)

Since air is assumed to be calorically perfect, it follows that high temperature effects ${ }^{63}$ are not captured within the model. As such, the combustor temperatures in Figure 4 (lower left) may be excessively large. Future work will consider high temperature gas effects within the combustor. This is important because material temperature limits within the combustor are stated as $4500^{\circ} R$ within ${ }^{64}$.

Trim Thrust Margin. From Figure 4 (lower right), we also observe that

- Trim thrust margin is a concave down function of $\left(h_{i}, A_{d}\right)$ - maximized at $h_{i} \approx 6, A_{d} \approx 0.125$.

Trim Elevator and AOA. Figure 5 shows how trim elevator and AOA depend on $\left(h_{i}, A_{d}\right)$. From Figure 5 , one observes that the:
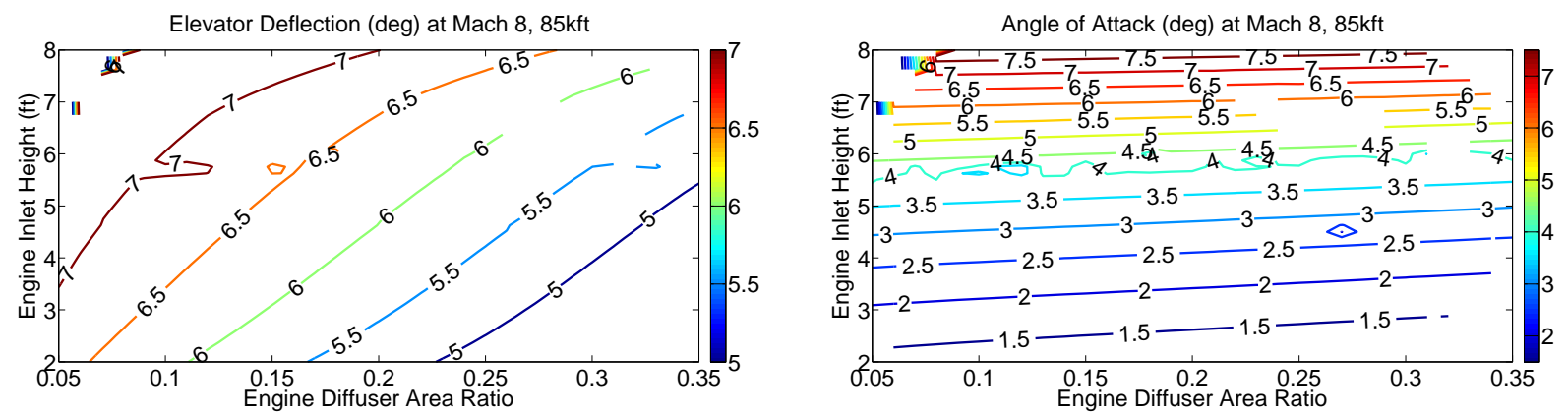

Figure 5. Trim Elevator Deflection and Trim AOA: Dependence on $\left(h_{i}, A_{d}\right)-$ Mach 8, 85 kft, Level Flight

- Trim elevator increases with increasing $h_{i}$ for a fixed $A_{d}$;

- Trim elevator increases with decreasing $A_{d}$ for a fixed $h_{i}$; 
- Trim AOA increases with increasing $h_{i}$ for fixed $A_{d}$. Trim AOA decreases with increasing $A_{d}$ for fixed $h_{i}$. (For $h_{i}$ sufficiently large, trim AOA becomes nearly independent of $A_{d}$.)

\section{III..2. Impact of Engine Parameters on Dynamic Properties (Mach 8, 85 kft, Level Flight)}

The following figure shows the impact of $h_{i}$ and $A_{d}$ on the vehicle instability and RHP transmission zero associated with FPA.
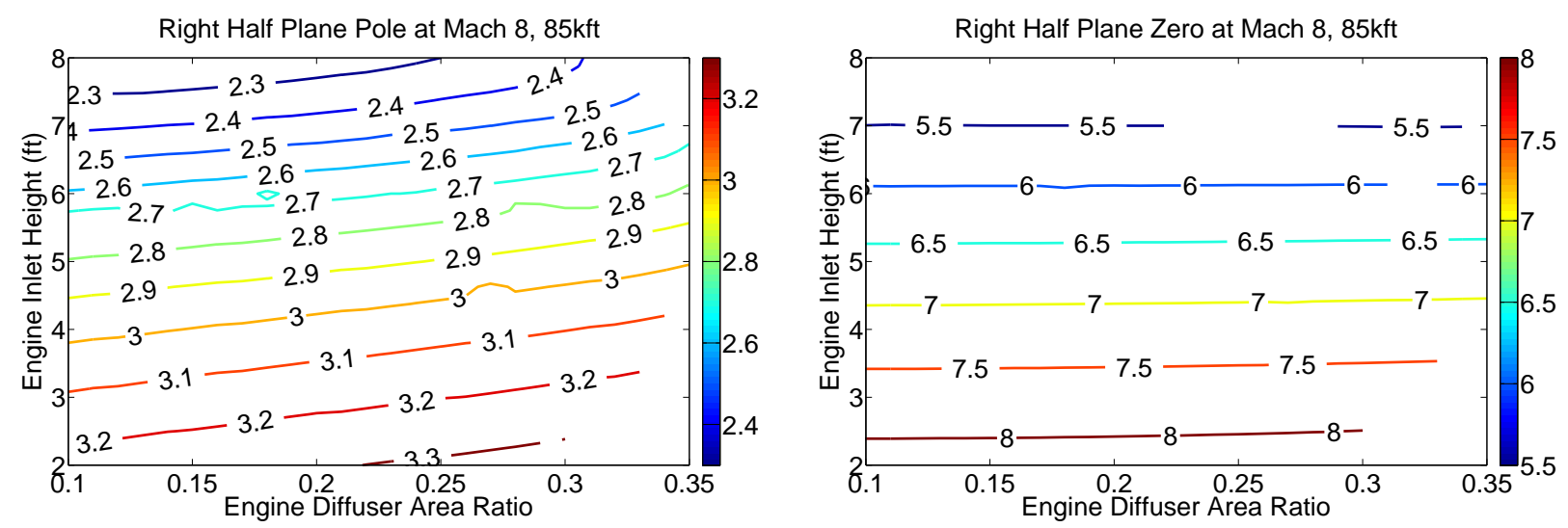

Figure 6. Right Half Plane Pole and Zero: Dependence on $\left(h_{i}, A_{d}\right)-$ Mach 8, 85 kft, Level Flight

From Figure 6, one observes that the:

- RHP pole increases with increasing $A_{d}$ (for a fixed $h_{i}$ ) and decreasing $h_{i}$ (for a fixed $A_{d}$ );

- RHP zero is constant with respect to $A_{d}$ (for a fixed $h_{i}$ ); it decreases with increasing $h_{i}$ (for a fixed $\left.A_{d}\right)$.

\section{III..3. Comparison of Engine Designs (Mach 8, $85 \mathrm{kft}$, Level Flight)}

In the previous sections, we considered the impact of increasing the engine height $h_{i}$ and diffuser area ratio $A_{d}$. We consider $h_{i} \leq 6$ (bound chosen due to combustor temperature effects) and $A_{d} \geq 0.125$ (bound chosen due to thrust margin effects). Within this range, we observe the following trade-offs:

- Increasing $h_{i}\left(\right.$ fixed $\left.A_{d}\right)$

- PROS: Trim FER reduces, trim combustor temperature decreases (till $h_{i} \approx 5.5$ at $\left.A_{d}=0.125\right)$, trim thrust margin increases, trim lift-to-drag increases (for $h_{i}>4.0$ at $A_{d}=0.125$, not shown), trim drag decreases (for $h_{i}>4.0$ at $A_{d}=0.125$, not shown), RHP pole reduces;

- CONS: Trim fuel rate increases, trim elevator increases, trim AOA increases, RHP zero decreases, trim lift-to-drag decreases (for $h_{i}<4.0$ at $A_{d}=0.125$, not shown), trim drag increases (for $h_{i}<4.0$ at $A_{d}=0.125$, not shown);

- Decreasing $A_{d}\left(\right.$ fixed $\left.h_{i}\right)$

- PROS: Trim FER decreases, trim fuel rate decreases, trim combustor temperature decreases, trim thrust margin increases, RHP pole decreases (marginally);

- CONS: Trim elevator increases, trim AOA increases (marginally), trim lift-to-drag decreases (not shown), trim drag increases (not shown).

Table 4 shows a comparison of the three engine designs described above. The first is the nominal engine design presented in ${ }^{7-9,19,26,28,36,38}$ As stated earlier, this configuration is geometrically unfeasible with respect to the implied flat base vehicle diagram shown in Figures 1 and 2. As can be seen from the table, it is generally "slow" with a small maximum acceleration capability. The second engine design will be used throughout the remainder of this paper. It satisfies each of the constraints listed at the beginning of Section III. The third configuration is a faster configuration that also obeys the constraints. 


\begin{tabular}{|c|c|c|c|c|c|c|c|c|c|}
\hline Engine & $h_{i}$ & $A_{d}$ & $A_{n}$ & $h_{e}$ & Trim L2D & Trim Fuel Rate & RHP Pole & RHP Zero & Z/P Ratio \\
\hline Nominal & $3.25 \mathrm{ft}$ & 1 & 6.35 & $5 \mathrm{ft}$ & 2.17 & 0.051 slugs $/ \mathrm{s}$ & 3.1 & 8.5 & 2.7 \\
New & $4.5 \mathrm{ft}$ & 0.15 & 6.67 & $4.5 \mathrm{ft}$ & 4.32 & 0.071 slugs $/ \mathrm{s}$ & 2.9 & 6.9 & 2.4 \\
Fast & $6 \mathrm{ft}$ & 0.125 & 8 & $6 \mathrm{ft}$ & 4.53 & 0.094 slugs $/ \mathrm{s}$ & 2.6 & 6.1 & 2.3 \\
\hline \hline Engine & Trim FER & \multicolumn{2}{|c|}{ Trim Temp. } & Trim Thrust & Trim Elev. & Trim AOA & Max Thrust & Max Acceleration \\
\hline Nominal & 0.47 & $4500^{\circ} \mathrm{R}$ & $1250 \mathrm{lbf}$ & $9.7^{\circ}$ & \multicolumn{2}{|c|}{$1^{\circ}$} & $2834 \mathrm{lbf}$ & $11.1 \mathrm{ft} / \mathrm{s}^{2}$ \\
New & 0.35 & $4750^{\circ} \mathrm{R}$ & $1380 \mathrm{lbf}$ & $6.25^{\circ}$ & $3.1^{\circ}$ & $4647 \mathrm{lbf}$ & $18.2 \mathrm{ft} / \mathrm{s}^{2}$ \\
Fast & 0.3 & $4400^{\circ} \mathrm{R}$ & $1445 \mathrm{lbf}$ & $6.9^{\circ}$ & $4.6^{\circ}$ & $6582 \mathrm{lbf}$ & $28.8 \mathrm{ft} / \mathrm{s}^{2}$ \\
\hline
\end{tabular}

Table 4. Comparison of 3 Engine Designs (Mach 8, 85 kft, Level Flight)

Table 4 shows that with respect to the nominal (slow or small) engine, the new (intermediately fast and sized) engine has the following associated PROS and CONS at Mach 8, $85 \mathrm{kft}$, level flight:

- PROS: smaller trim elevator, smaller trim FER, larger maximum thrust, larger thrust margin, larger maximum acceleration, smaller RHP pole;

- CONS: larger engine, larger mass, larger trim thrust, larger trim combustor temperature, larger trim AOA, smaller RHP zero, smaller RHP zero-pole ratio.

Future work will examine the above tradeoffs more precisely.

\section{Vehicle Parameter Studies}

This section examines the impact of the following vehicle parameters:

- Engine location with respect to vehicle nose, lower forebody compression ramp inclination, center of gravity $(\mathrm{CG})$ location with respect to vehicle nose, and vehicle mass

across the vehicle's level-flight trimmable region.

\section{IV.A. Engine Location with respect to Vehicle Nose}

In this section, we examine the impact of moving the engine rearward with respect to the vehicle nose. The following assumptions will be made:

- The new engine parameters are being used; i.e. $h_{e}=h_{i}=4.5 \mathrm{ft}, A_{d}=0.15, A_{n}=\frac{1}{A_{d}}=6.67$.

- The engine location is varied between 40 and $60 \mathrm{ft}$ from the vehicle nose.

- The vehicle's center of gravity $(\mathrm{cg})$ moves with the engine location (assumed fixed near engine combustor).

\section{IV.A.1. Impact of Engine Location on Static Properties (Level Flight)}

Figure 7 shows how the trimmable region changes with the engine location. Specifically, it shows that the trimmable region shrinks as the engine is shifted rearward.

Trim AOA. From Figure 8, we observe the following:

- Trim AOA decreases as the engine is moved rearward for a fixed Mach and altitude.

- For a fixed altitude, trim AOA dependence on engine location is a bit complex. When the engine is closer to the nose, trim AOA increases with Mach. This is because a forward situated engine results in a forward CG shift and hence a more stable aircraft. When the engine is moved rearward, trim AOA becomes insensitive to Mach variations. This dependence requires further examination.

- For a fixed engine location and Mach, trim AOA increases with increasing altitude. 


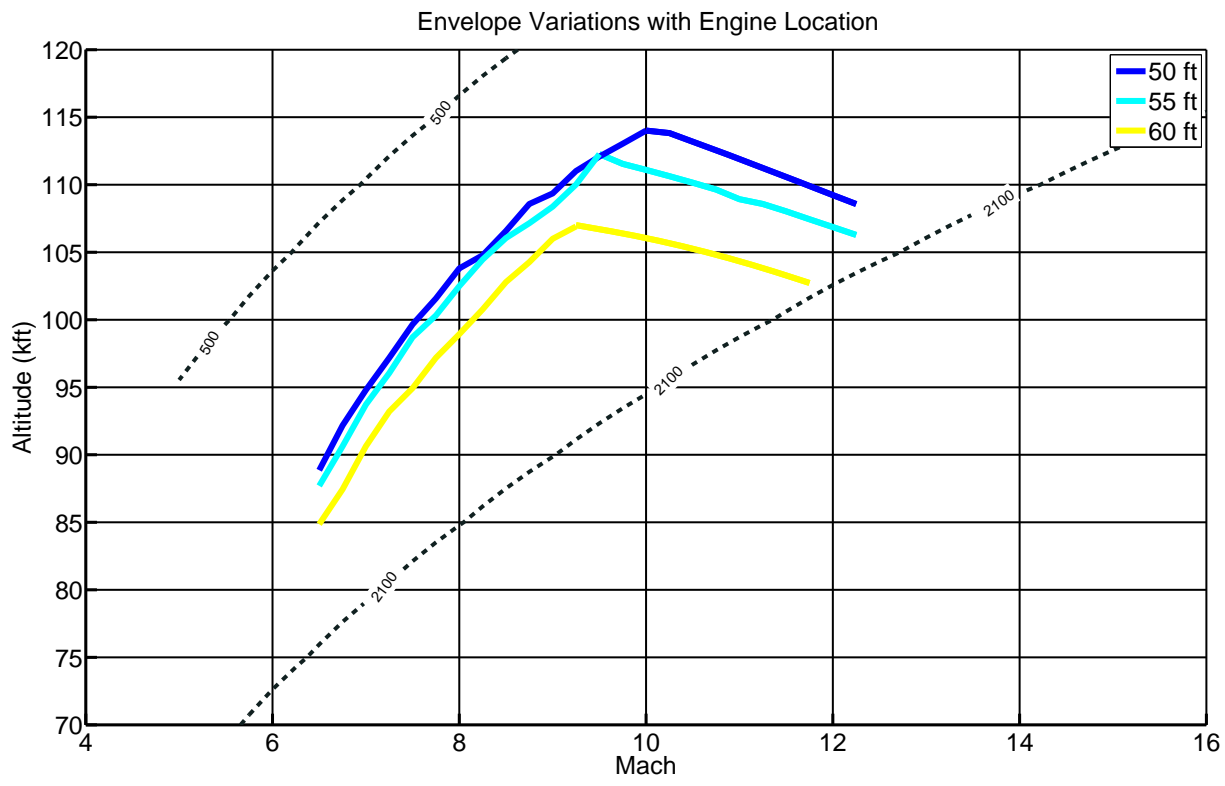

Figure 7. Impact of Engine Location on (Level Flight) Trimmable Region
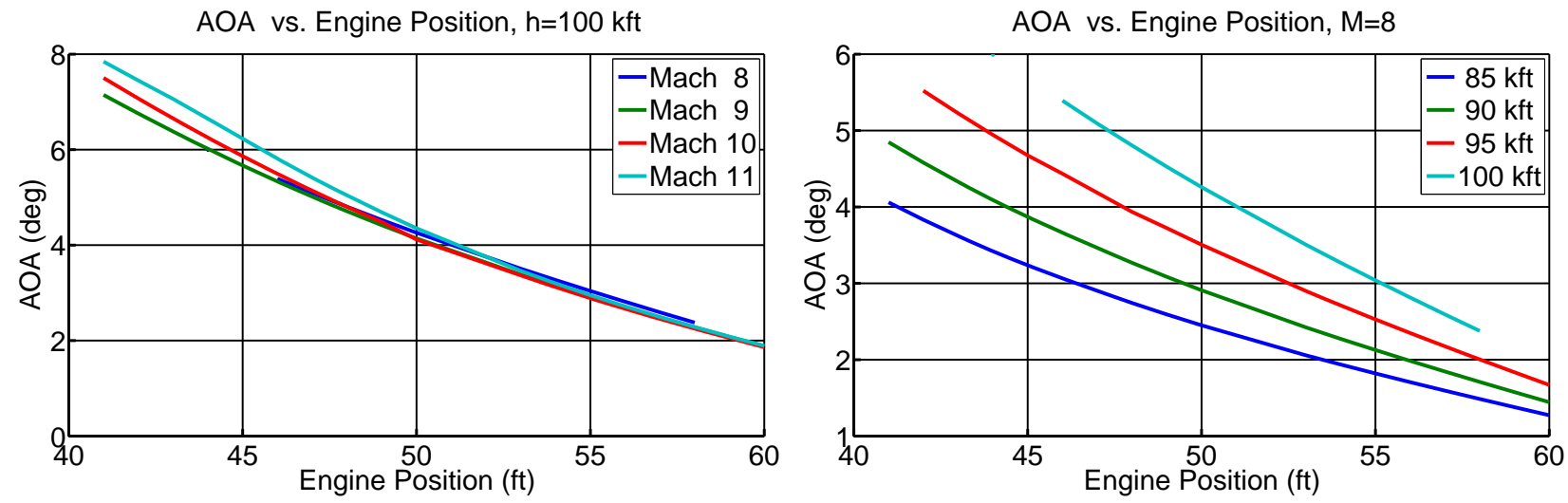

Figure 8. Impact of Engine Location on Trim AOA

A smaller AOA is typically desirable when a designer wishes to increase the vehicle's stall margin. (Generally speaking, an AOA that is too large results in flow separation and loss of lift.)
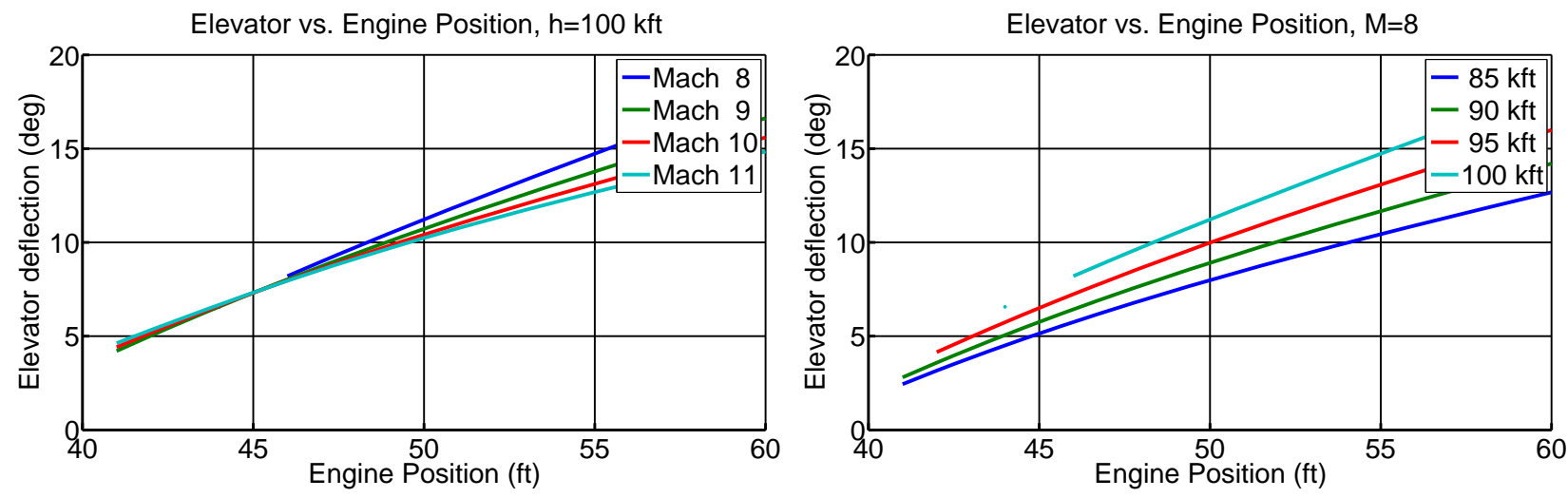

Figure 9. Impact of Engine Location on Trim Elevator 
Trim Elevator. From Figure 9, we observe the following:

- The trim elevator deflection increases as the engine is moved rearward for a fixed Mach and altitude.

- For a fixed altitude, the dependence of trim elevator on engine location is nearly linear with the slope decreasing with increasing Mach. When the engine is closer to the nose (more stable vehicle), trim elevator increases with increasing Mach. When the engine is closer to the rear (more unstable vehicle), trim elevator increases with decreasing Mach. From this, it follows that flight at a fixed altitude and a low (high) Mach requires less (more) elevator for a forward situated engine (more stable vehicle), and more (less) elevator for a rearward situated engine (more unstable vehicle).

- For a fixed Mach, the dependence of trim elevator on engine location is almost linear with the slope increasing slightly with increasing altitude. When the engine location is also fixed, the trim elevator increases with increasing altitude.

- Trim elevator deflection increases with increasing altitude.

Trim FER. Figure 10 illustrates how trim FER depends on engine location. From the figure, the following
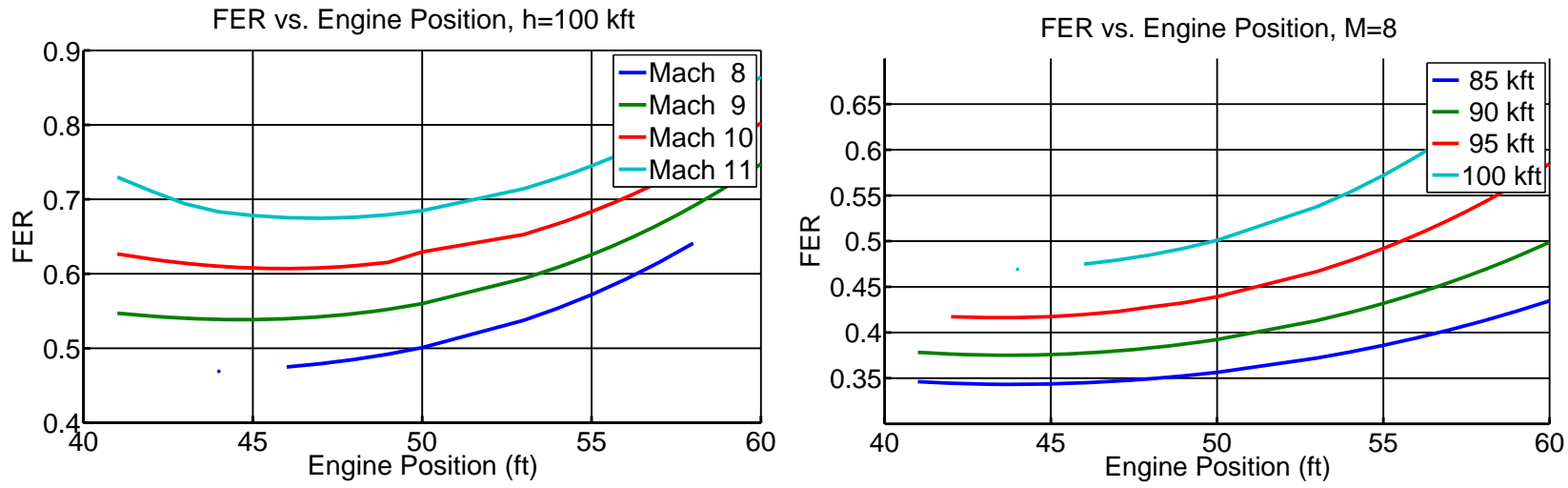

Figure 10. Impact of Engine Location on Trim FER

is observed:

- Trim FER is a concave up function with respect to engine location for a fixed altitude and Mach - with trim FER being minimized near $45 \mathrm{ft}$ for most flight conditions.

- For a fixed engine location and altitude (or Mach), trim FER increases with increasing Mach (or altitude).

\section{IV.A.2. Impact of Engine Location on Dynamic Properties (Level Flight)}

RHP Pole vs. Engine Position, $\mathrm{h}=100 \mathrm{kft}$

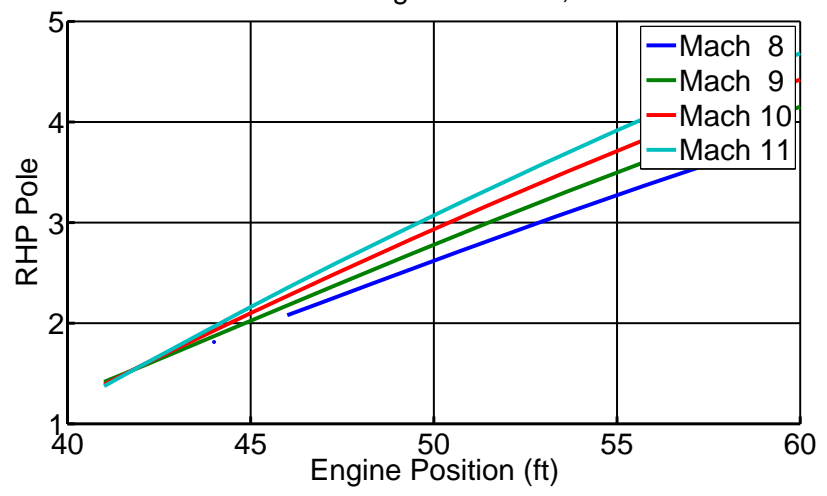

RHP Pole vs. Engine Position, $M=8$

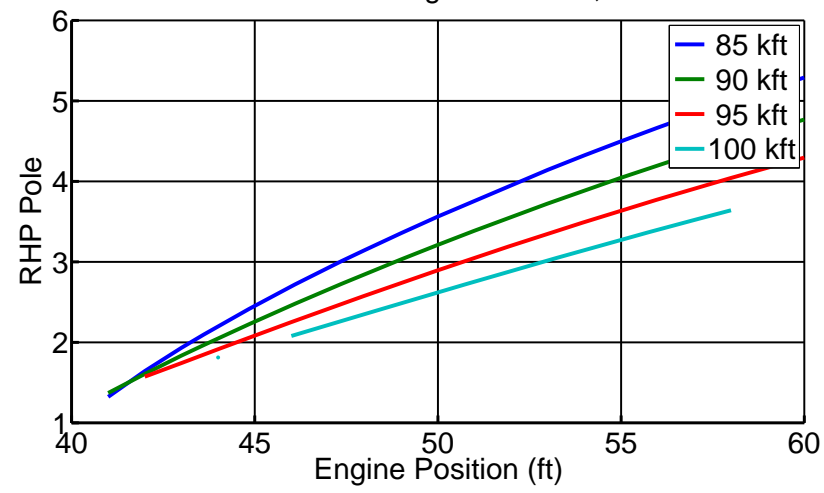

Figure 11. Impact of Engine Location on Right Half Plane Pole

RHP Pole. Figure 11 shows that the 
- instability increases (roughly linearly) as the engine is moved rearward;

- instability increases with increasing Mach and decreasing altitude.

Moving the engine rearward, moves the center of gravity (cg) rearward with respect to the aerodynamic center (ac) - thus making the airplane more unstable.

Motivation for Increased Instability. From the above, a designer may wish to increase the vehicle instability in order to make the vehicle more maneuverable in terms of following aggressive flight path angle or vertical acceleration commands. It may also be desirable in order to facilitate the attenuation of high frequency wind disturbances. (The link between instability and maneuverability was understood by the Wright Brothers early on in their work [65, page 39]. This could be important for a missile going after agile targets. Such might be the case for military applications. In such a case, one should note that a larger instability requires a larger minimum control system bandwidth for vehicle stabilization. ${ }^{41}$ This, however, may conflict with higher frequency non-minimum phase, structural, aero-elastic, and actuator dynamics. In the same spirit, a larger bandwidth at the elevator would typically require a faster control surface actuator. Such considerations must be rigorously addressed at some point in the design process - the sooner, the better.
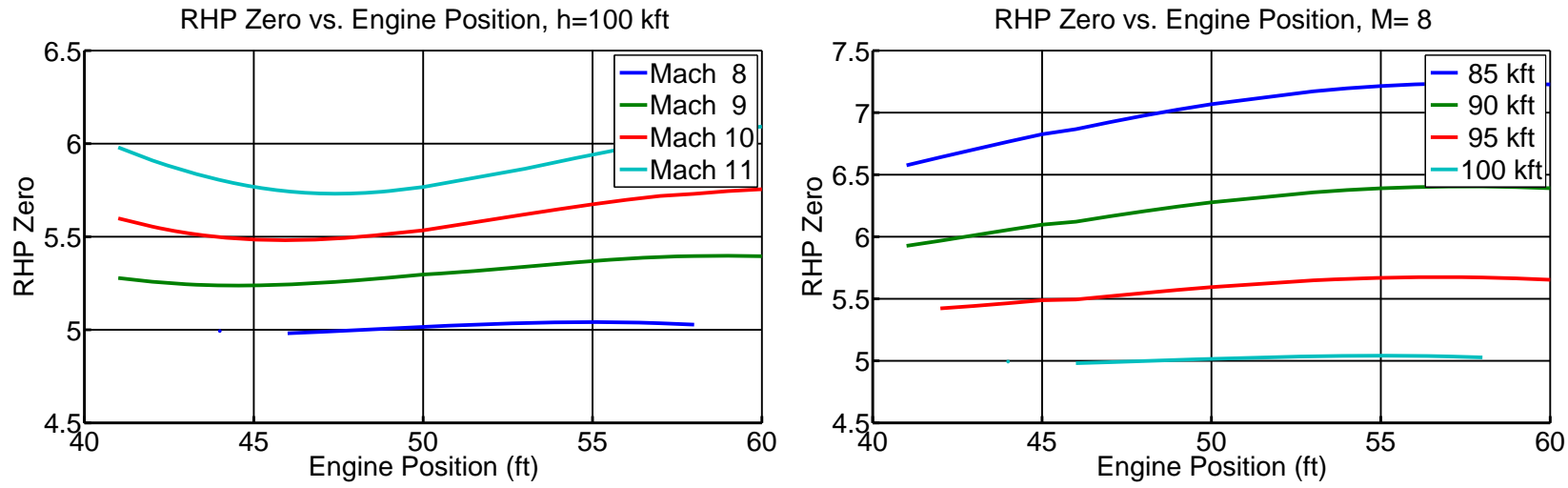

Figure 12. Impact of Engine Location on Right Half Plane Zero

RHP Zero. From Figure 12, one observes that the:

- RHP zero varies little with engine position for a fixed altitude and Mach

- RHP zero increases with increasing Mach and decreasing altitude

Increasing RHP Zero: Moving Engine Rearward. From the above, it follows that one might move the engine rearward (making the vehicle more unstable) in order to maximize the right half plane zero. By so doing, a vehicle designer can (in principle) increase the maximum achievable flight path angle bandwidth. ${ }^{41}$ One must, of course, note that flexible modes, the associated uncertainty, and the control system simplicity can also limit the achievable bandwidth. This will be the case when the flexible modes lie within a decade of the right half plane zero. Additional pros associated with moving the engine rearward include: less fuel usage - minimized near $55 \mathrm{ft}$ (not shown). Associated cons include the following: trim L/D drops (monotonically for 40 to $60 \mathrm{ft}$ interval), trim FER increases (FER/thrust/acceleration margin decreases).

\section{IV.B. Lower Forebody Inclination}

In this section, we examine the impact of varying the lower forebody inclination angle. The following is assumed:

- New engine parameters; i.e. $h_{e}=h_{i}=4.5 \mathrm{ft}, A_{d}=0.15, A_{n}=\frac{1}{A_{d}}=6.67$.

- Lower forebody inclination varied from $4.2^{\circ}$ to $8.2^{\circ}\left(\right.$ nominal value $\left.=6.2^{\circ}\right)$

- All lengths (forebody, aftbody, engine length), upper forebody angle kept constant 
- Tail angle and total vehicle height change as a result

- CG assumed to be fixed

- Heating effects due to slender nose are not considered

\section{IV.B.1. Impact of Lower Forebody Inclination on Static Properties (Level Flight)}

Trimmable Region. Figure 13 shows how the trimmable region changes with lower forebody inclination angle. From Figure 13, one observe that the:

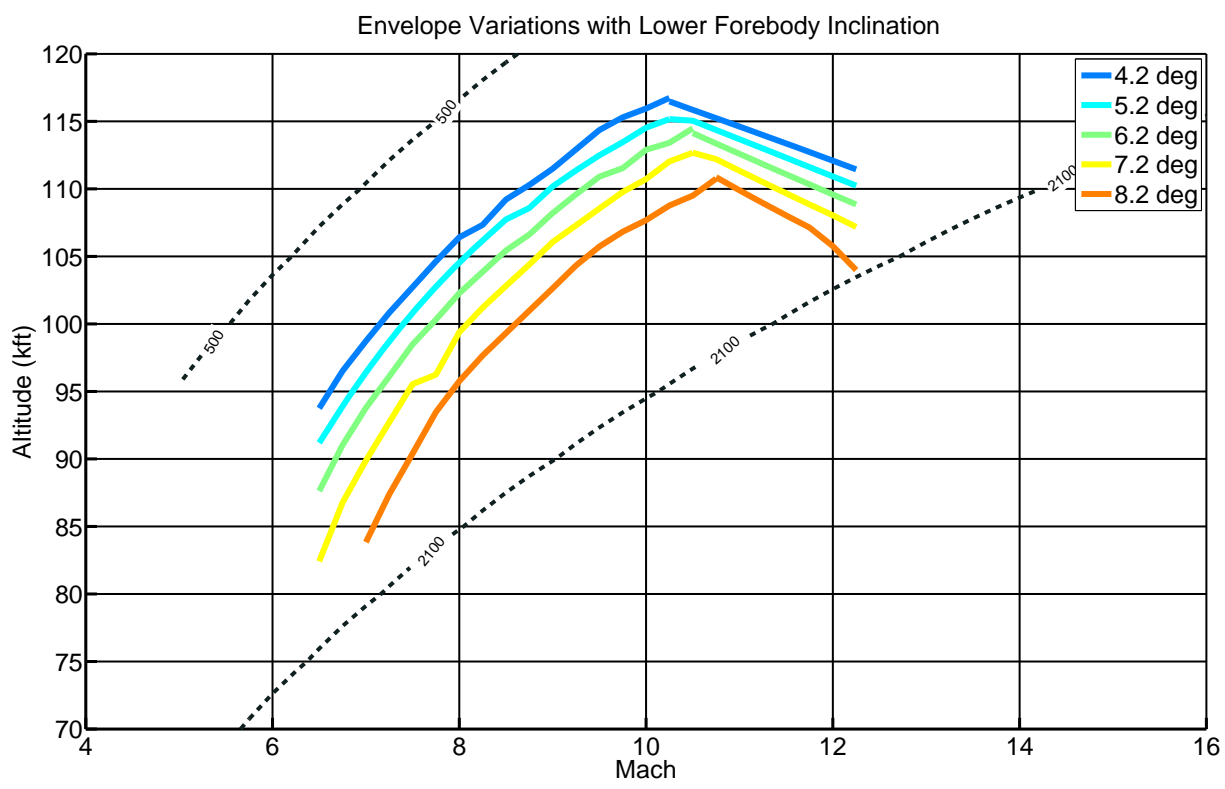

Figure 13. Impact of Lower Forebody Inclination on (Level Flight) Trimmable Region

- Trimmable region shrinks with increasing lower forebody inclination

- Pinch point moves toward a higher Mach and lower altitude with increasing lower forebody inclination

Trim AOA. Figure 14 shows how AOA varies with lower forebody inclination angle. From Figure 14, one observes that the:
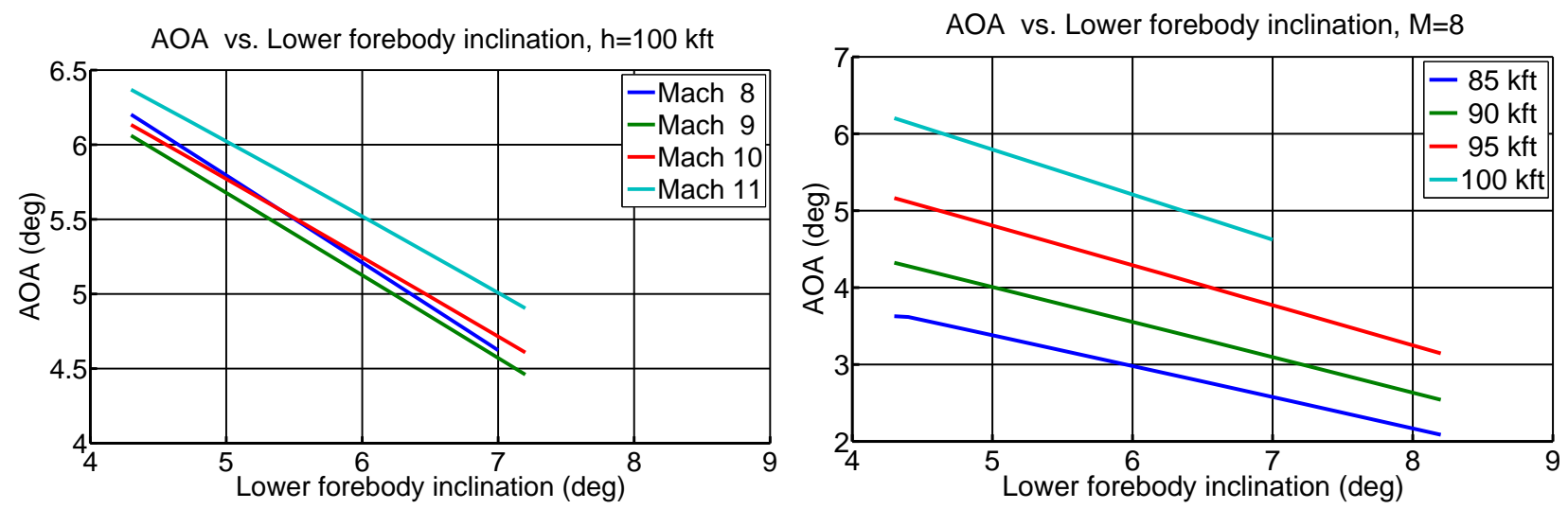

Figure 14. Impact of Lower Forebody Inclination on Trim AOA

- Trim AOA decreases linearly with increasing lower forebody inclination for a fixed altitude 
- Trim AOA decreases with Mach at lower Mach numbers for a fixed altitude;

- Trim AOA increases with increasing altitude for a fixed Mach

Trim Elevator. Figure 15 shows how elevator varies with lower forebody inclination angle. From Figure 15, one observes that the:
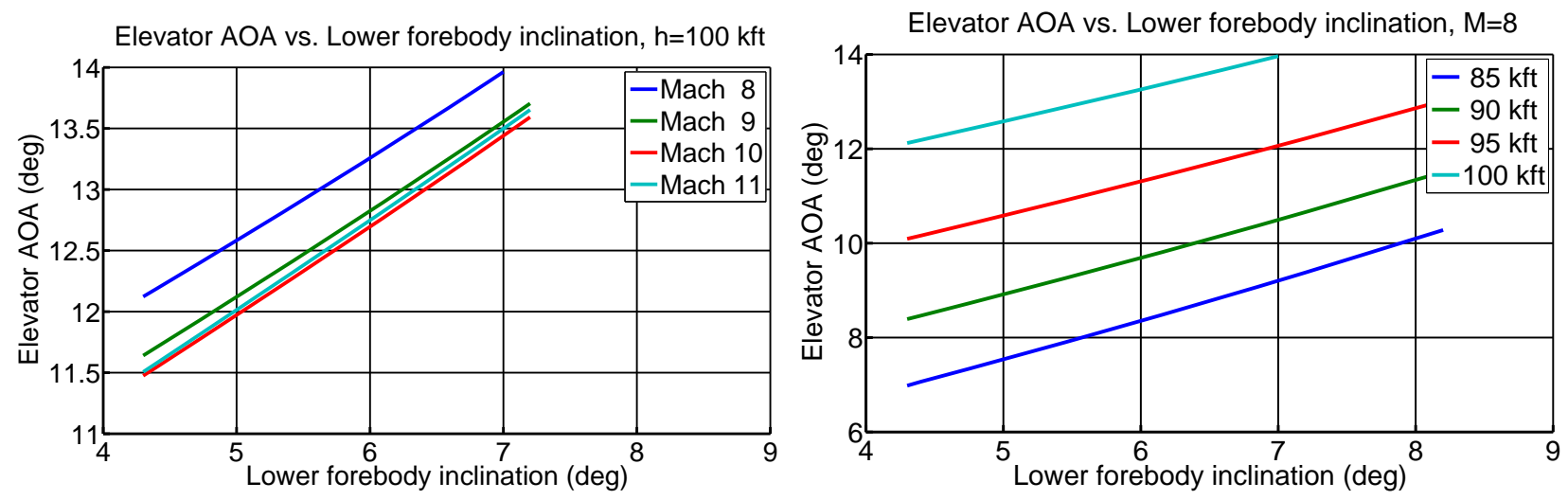

Figure 15. Impact of Lower Forebody Inclination on Trim Elevator

- Trim elevator deflection increases linearly with increasing forebody inclination

- Trim elevator deflection increases with increasing Mach, increasing altitude

Trim FER. Figure 16 shows how FER varies with lower forebody inclination angle.
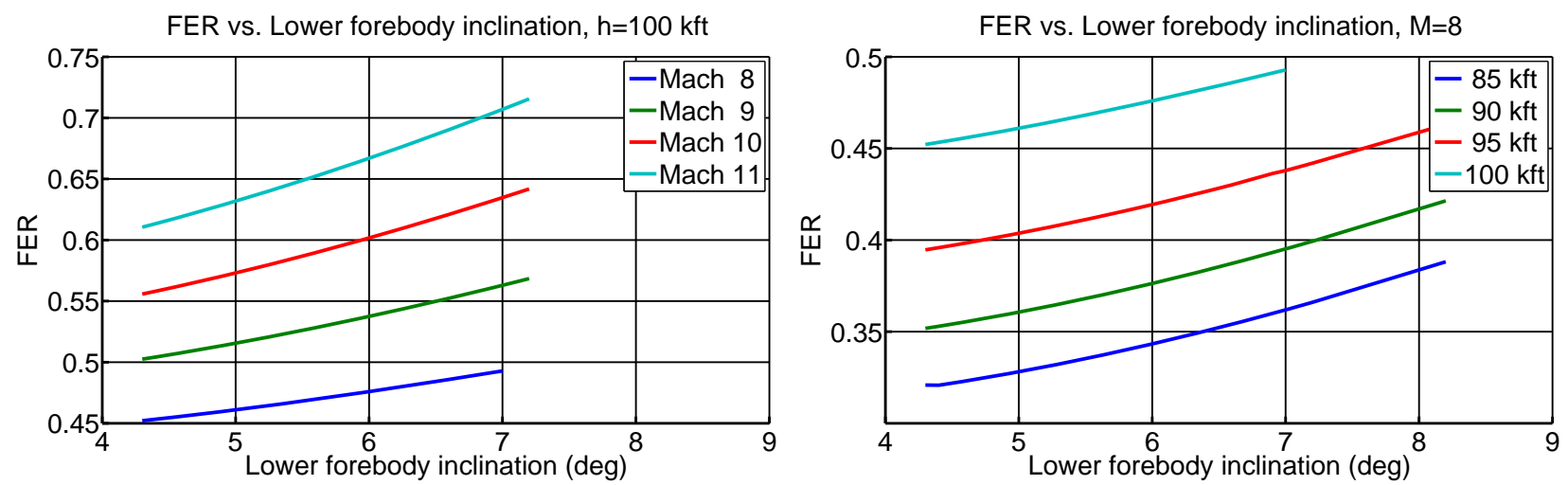

Figure 16. Impact of Lower Forebody Inclination on Trim FER

From Figure 16, one observes that the:

- Trim FER increases almost linearly with increasing lower forebody inclination

\section{IV.B.2. Impact of Lower Forebody Inclination on Dynamic Properties (Level Flight)}

RHP Pole. Figure 17 shows how the RHP pole varies with lower forebody inclination. From Figure 17, one observes that the:

- RHP pole increasing with increasing lower forebody inclination

- RHP pole increases with Mach and decreasing altitude

RHP Zero. Figure 18 shows how the RHP pole varies with lower forebody inclination. From Figure 18, one observes that the:

- RHP zero increases with increasing lower forebody inclination

- RHP zero increases with increasing Mach and decreasing altitude 
RHP Pole vs. Lower forebody inclination, $h=100 \mathrm{kft}$

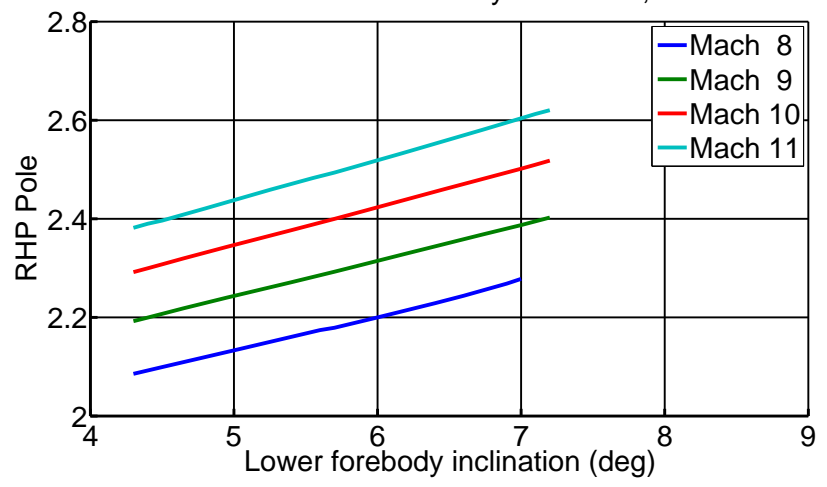

RHP Pole vs. Lower forebody inclination, $M=8$

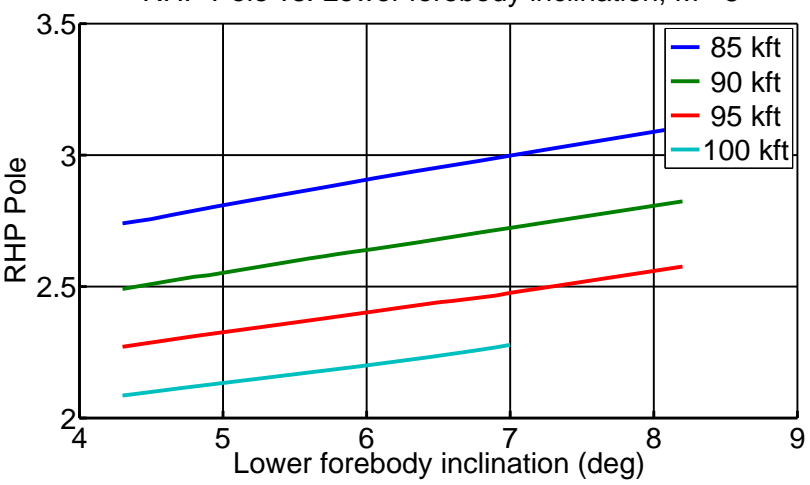

Figure 17. Impact of Lower Forebody Inclination on Right Half Plane Pole

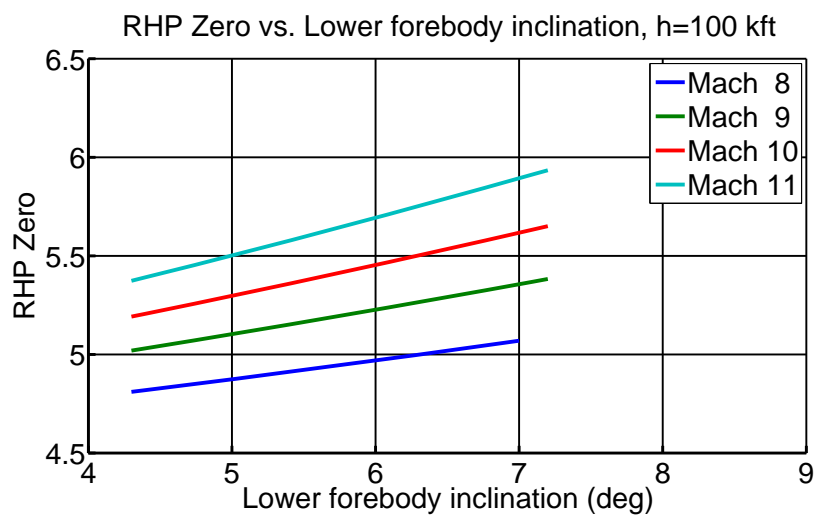

RHP Zero vs. Lower forebody inclination, $M=8$

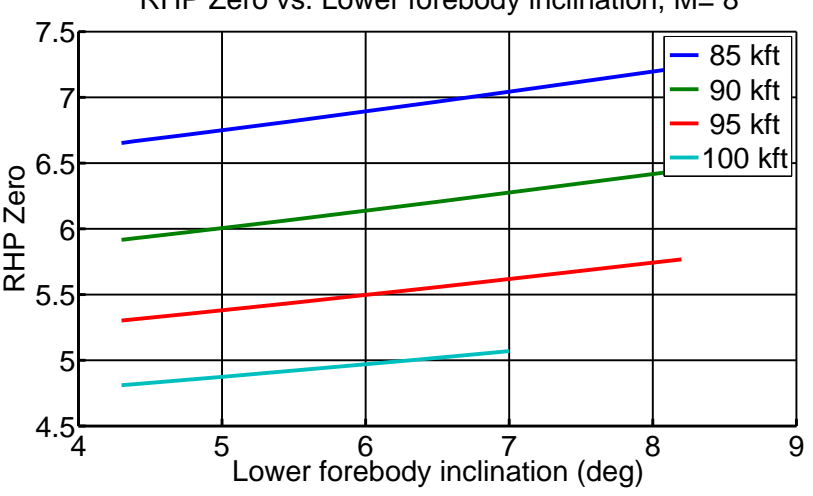

Figure 18. Impact of Lower Forebody Inclination on Right Half Plane Zero

\section{IV.C. Center of Gravity}

This section examines the impact of varying the center of gravity $(\mathrm{cg})$ location. The following assumptions are made:

- New engine parameters; i.e. $h_{e}=h_{i}=4.5 \mathrm{ft}, A_{d}=0.15, A_{n}=\frac{1}{A_{d}}=6.67$.

- CG varied from $45 \mathrm{ft}$ to $65 \mathrm{ft}$ artificially (no internal changes are made to achieve this shift)

- For dynamic properties, we only consider CG locations for which vehicle is open loop unstable

\section{IV.C.1. Impact of Center of Gravity on Static Properties (Level Flight)}

Trimmable Region. Figure 19 shows how the trimmable region changes with a shifting vehicle CG. From Figure 19, one observes that the

- trimmable region decreases slightly as the vehicle CG is moved rearward.

Trim AOA. Figure 20 shows how trim AOA depends on the vehicle's CG location.

Figure 20 shows that the

- Trim AOA decreases as the vehicle CG moves rearward.

Trim Elevator. Figure 21 shows how trim elevator depends on cg location.

- Trim elevator deflection increases as the vehicle CG moves rearward.

Trim FER. Figure 22 shows how trim FER depends on vehicle CG location. Figure 22 shows that the

- Trim FER increases as the vehicle CG moves rearward. 


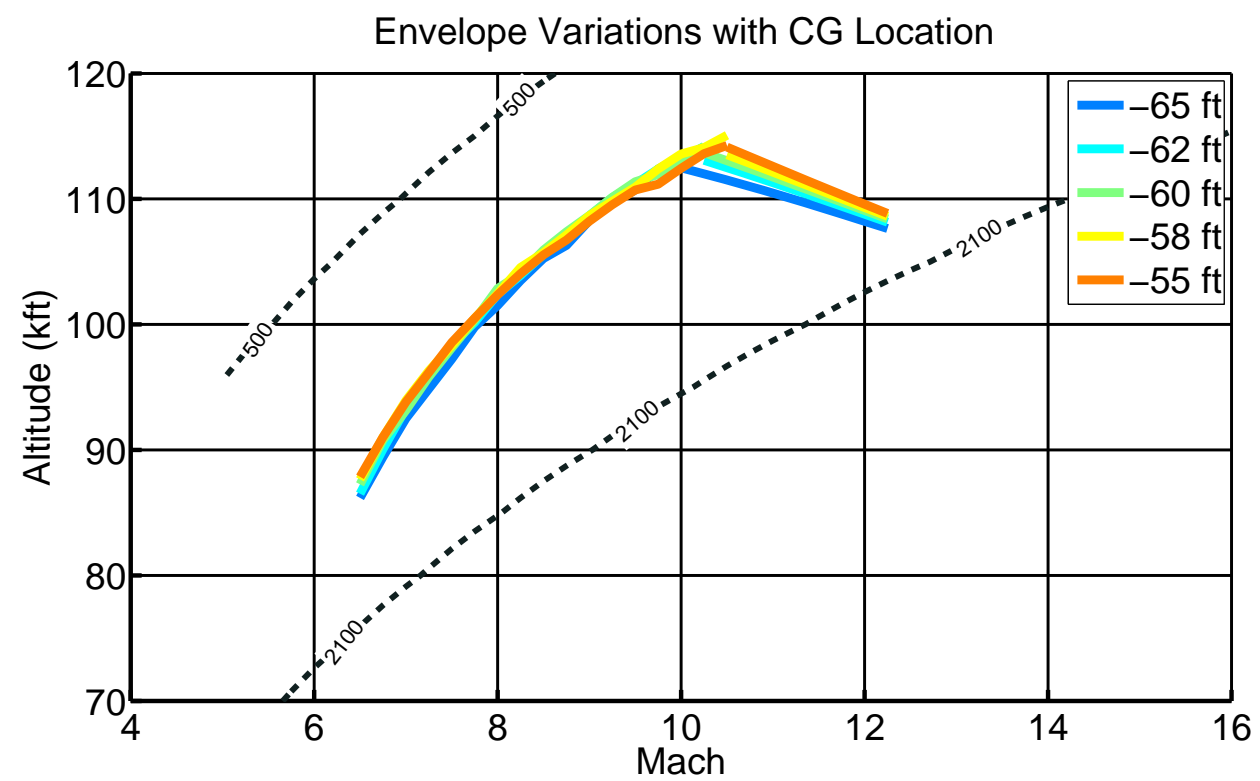

Figure 19. Impact of Vehicle CG Location on (Level Flight) Trimmable Region
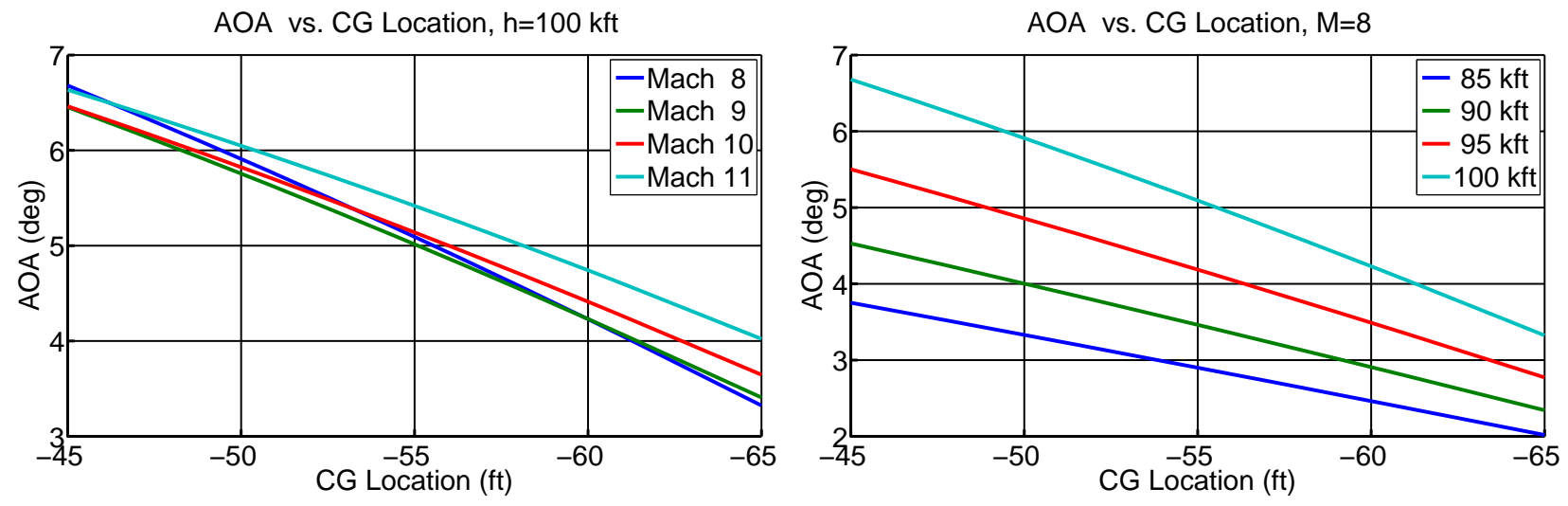

Figure 20. Impact of Vehicle CG Location on Trim AOA
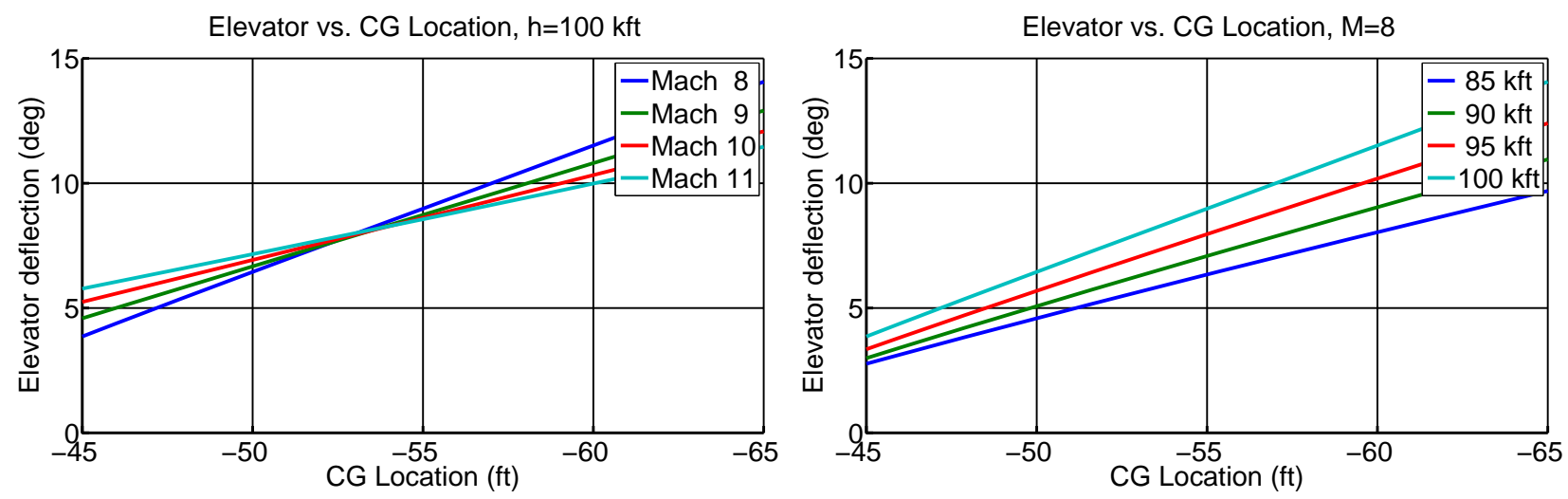

Figure 21. Impact of Vehicle CG Location on Trim Elevator

\section{IV.C.2. Impact of Center of Gravity on Dynamic Properties (Level Flight)}

RHP Pole. Figure 23 shows how the vehicle instability depends on the CG location. Figure 23 shows that the vehicle 
FER vs. CG Location, $\mathrm{h}=100 \mathrm{kft}$

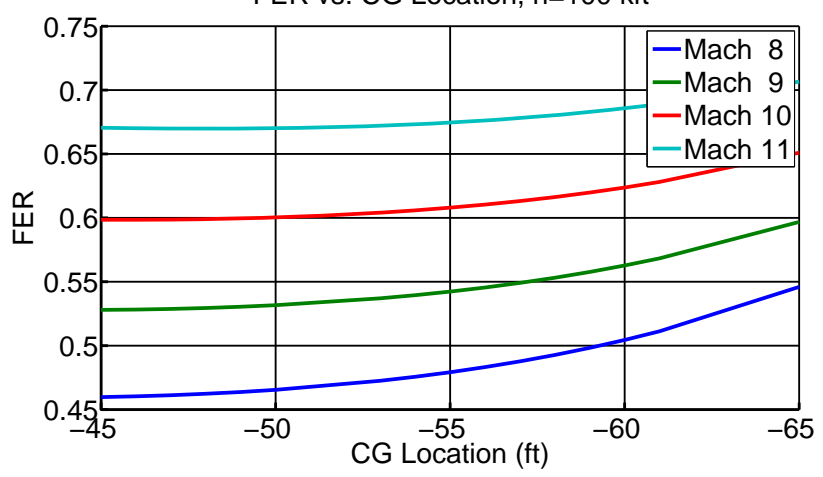

FER vs. CG Location, $M=8$

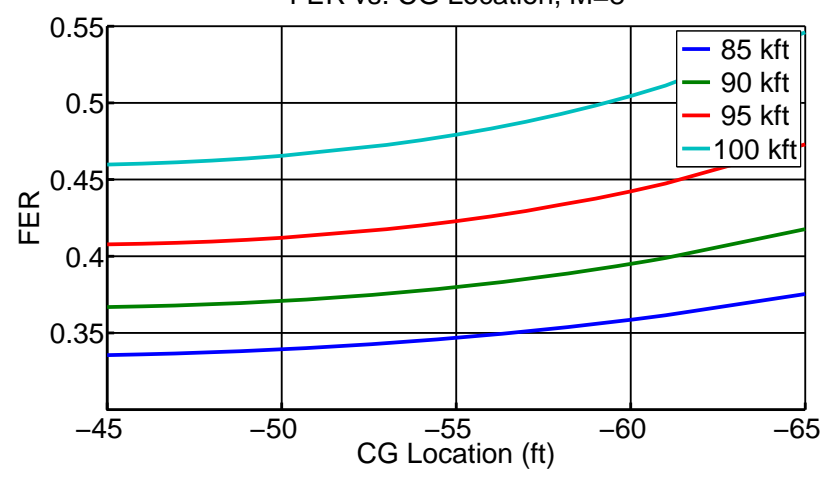

Figure 22. Impact of Vehicle CG Location on Trim FER

RHP Pole vs. CG Location, $h=100 \mathrm{kft}$

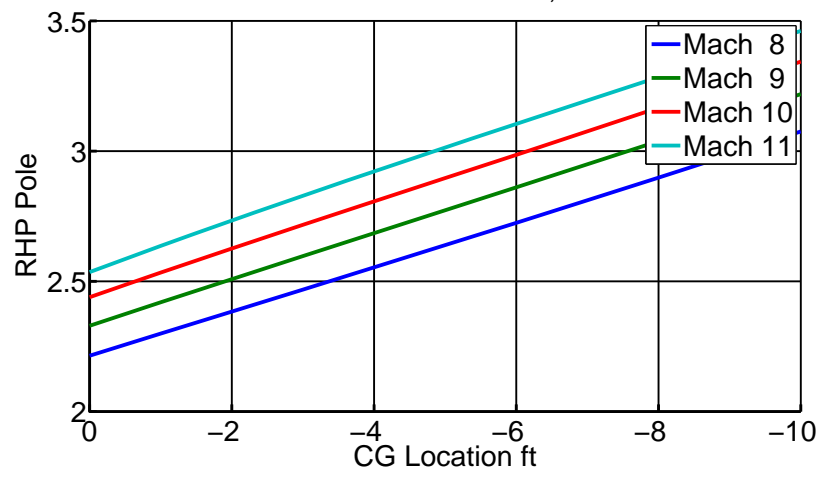

RHP Pole vs. CG Location, $M=8$

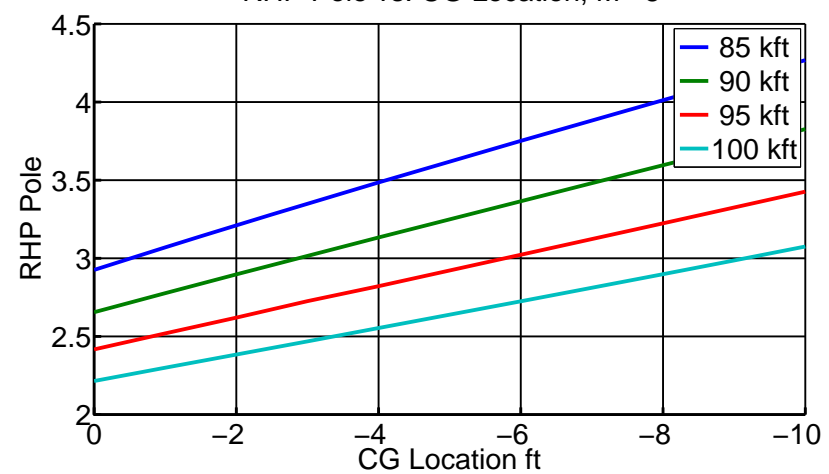

Figure 23. Impact of Vehicle CG Location on Right Half Plane Pole

- RHP pole increases linearly as the vehicle CG moves rearward.

RHP Zero. Figure 24 shows how the RHP zero depends on the CG location. Figure 24 shows that the vehicle
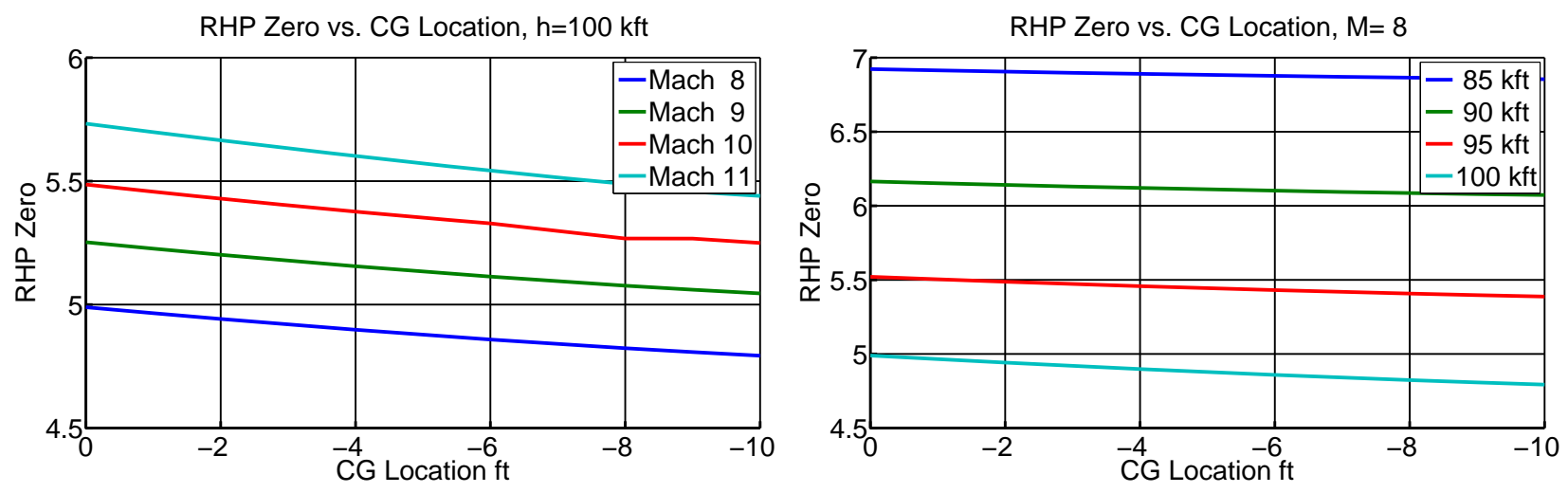

Figure 24. Impact of Vehicle CG Location on Right Half Plane Zero

- RHP zero decreases linearly as the vehicle CG moves rearward.

\section{IV.D. Vehicle Mass}

This section examines the impact of varying the vehicle's (total) mass. The following assumptions are made:

- New engine parameters; i.e. $h_{e}=h_{i}=4.5 \mathrm{ft}, A_{d}=0.15, A_{n}=\frac{1}{A_{d}}=6.67$. 
- Mass of vehicle modified without changing any material or subsystem properties.

\section{IV.D.1. Impact of Vehicle Mass on Static Properties}

Trimmable Region. Figure 25 shows how the trimmable region depends on vehicle mass.

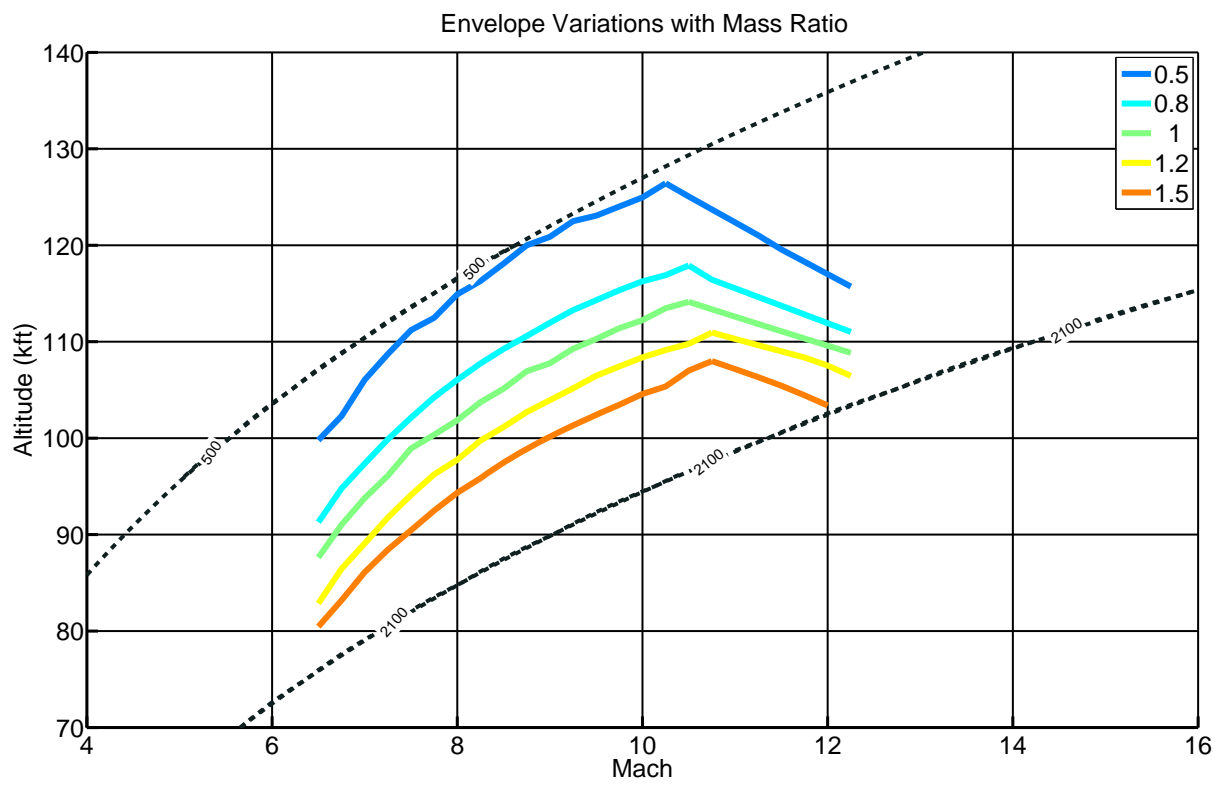

Figure 25. Impact of Vehicle Mass on (Level Flight) Trimmable Region

From Figure 25, one observes that the:

- Trimmable region shrinks as the vehicle mass is increased.

Trim AOA. Figure 26 shows how trim AOA depends on vehicle mass. The figure shows that the:
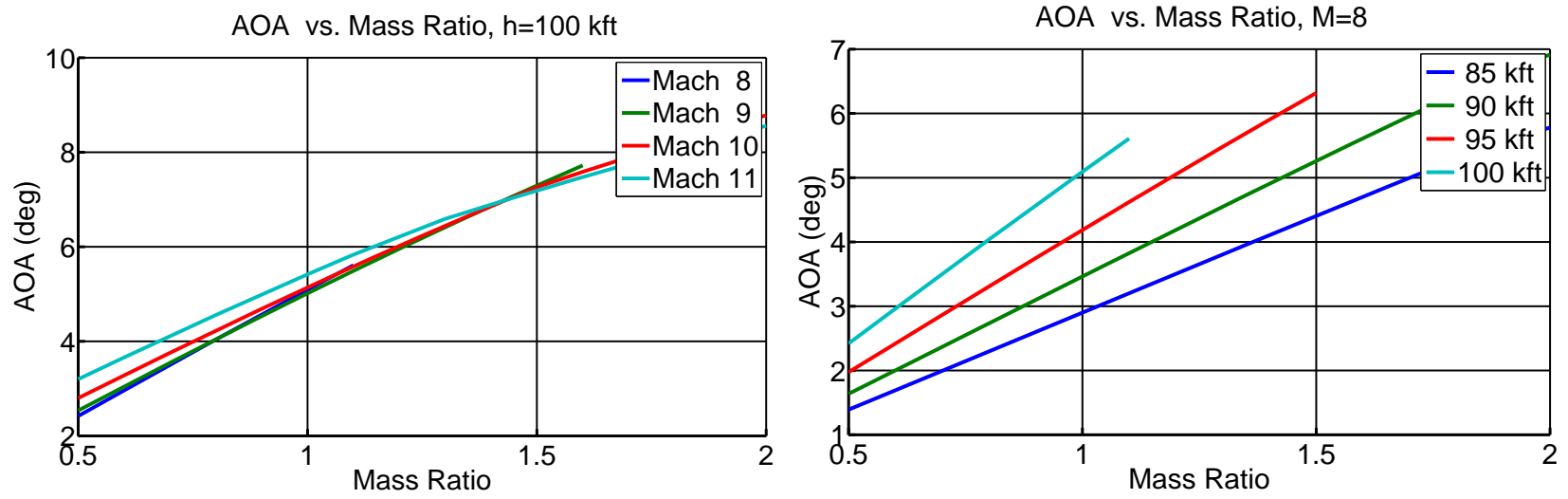

Figure 26. Impact of Vehicle Mass on Trim AOA

- Trim AOA increases as the vehicle mass is increased.

Trim Elevator. Figure 27 shows how trim elevator depends on vehicle mass. The figure shows that the:

- Trim elevator deflection increases as the vehicle mass is increased.

Trim FER. Figure 28 shows how trim FER depends on vehicle mass. The figure shows that the:

- Trim FER increases as the vehicle mass is increased. 
Elevator vs. Mass Ratio, $\mathrm{h}=100 \mathrm{kft}$

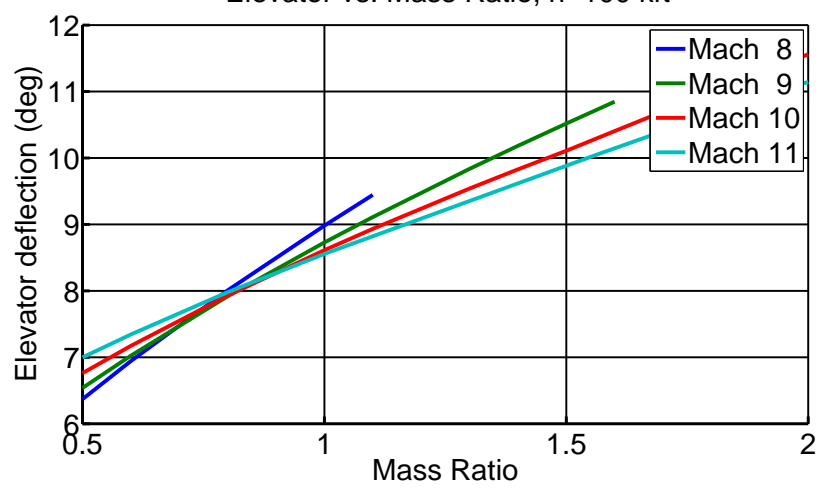

Elevator vs. Mass Ratio, $M=8$

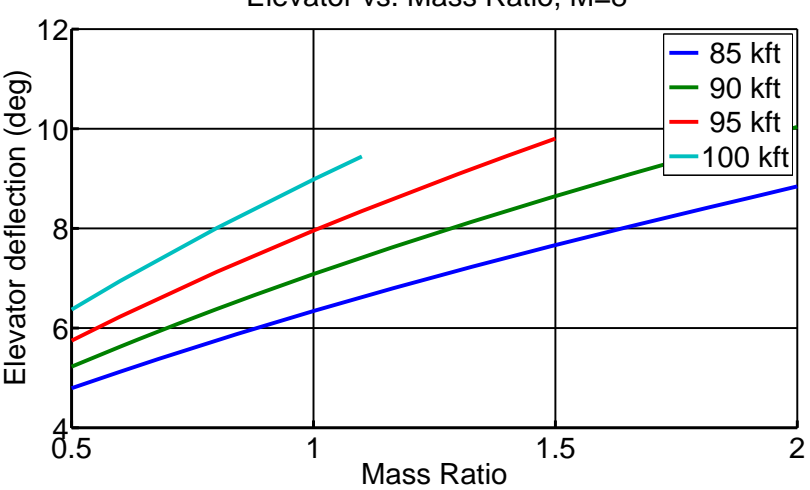

Figure 27. Impact of Vehicle Mass on Trim Elevator

FER vs. Mass Ratio, $\mathrm{h}=100 \mathrm{kft}$

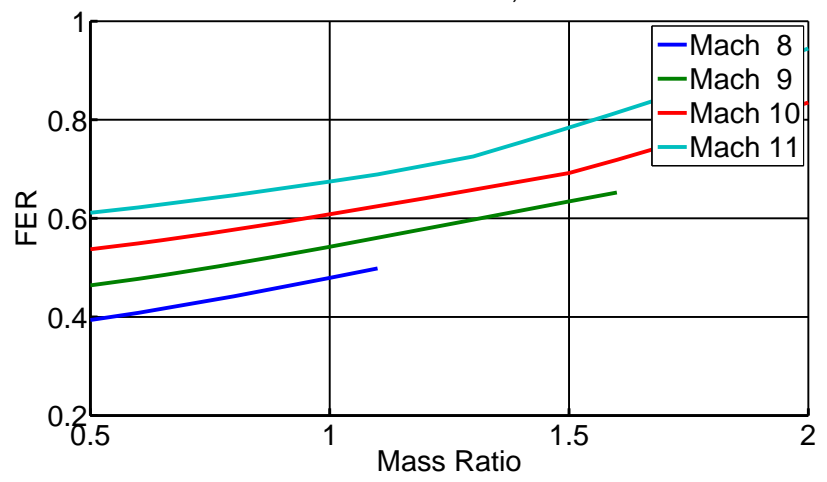

FER vs. Mass Ratio, $M=8$

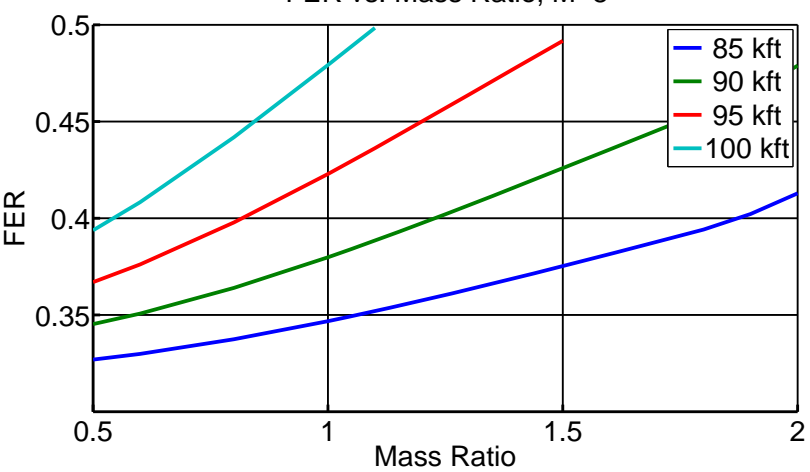

Figure 28. Trim FER vs Vehicle Mass

IV.D.2. Impact of Vehicle Mass on Dynamic Properties (Level Flight)

RHP Pole. Figure 29 shows how the vehicle instability depends on vehicle mass. The figure shows that the:
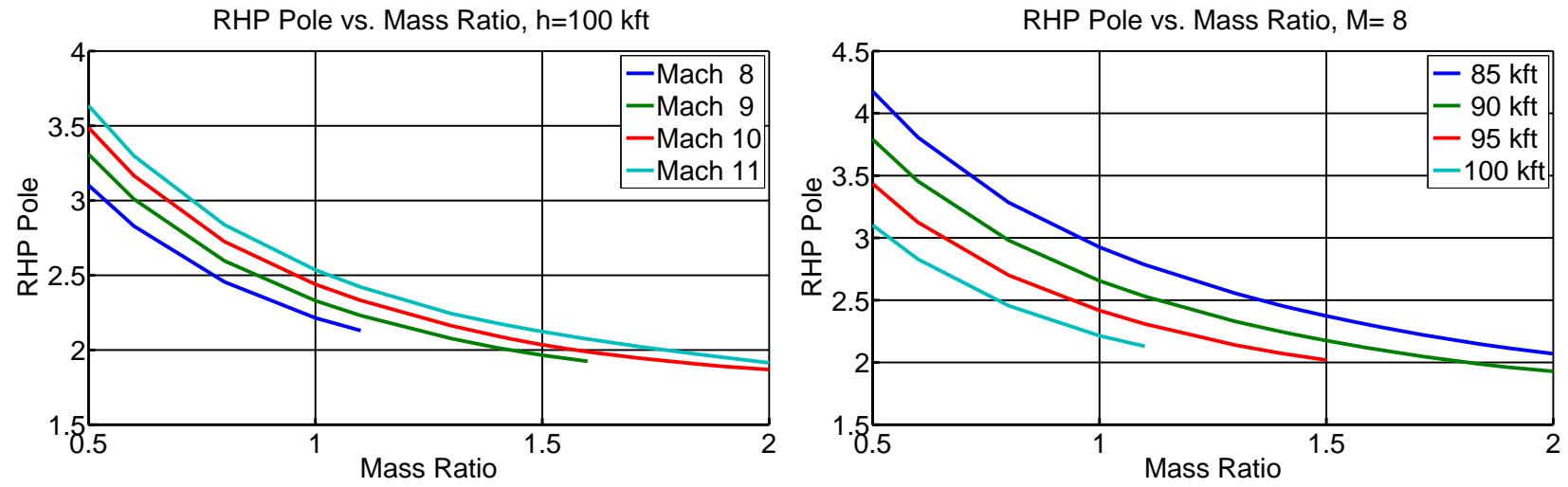

Figure 29. Impact of Vehicle Mass on Right Half Plane Pole

- RHP pole decreases as the vehicle mass is increased.

RHP Zero. Figure 30 shows how the RHP zero depends on vehicle mass. The figure shows that the:

- RHP zero decreases as the vehicle mass is increased. 
RHP Zero vs. Mass Ratio, $h=100$ kft

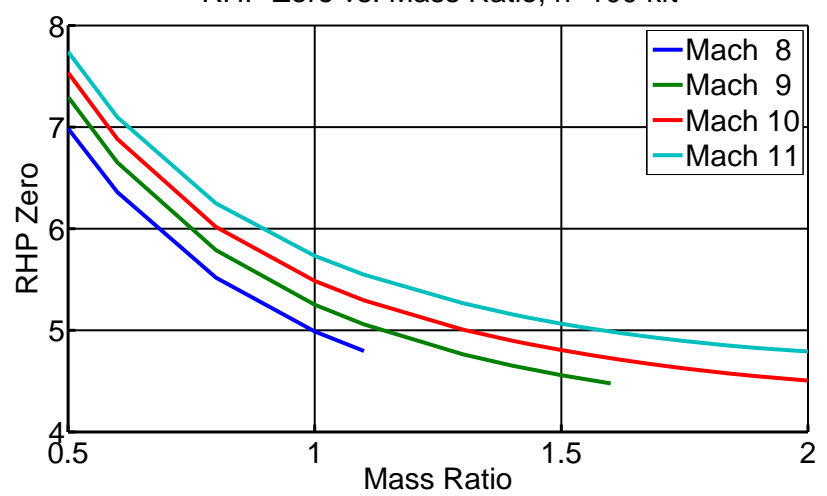

RHP Zero vs. Mass Ratio, $M=8$

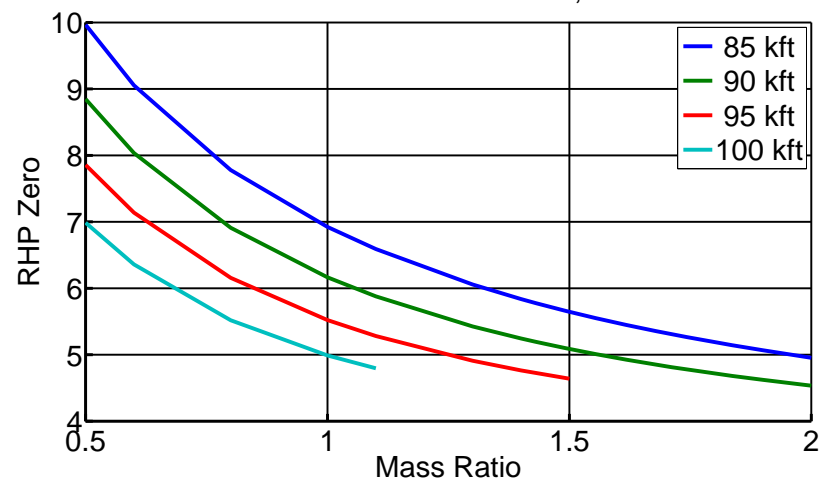

Figure 30. Impact of Vehicle Mass on Right Half Plane Zero

\section{IV.E. Summary of Level Flight Trim Trade Studies}

The following tables summarize each of the conducted level-flight trim trade studies.

Rearward Engine Shift. Table 5 summarizes trends for a rearward shifting engine. The engine is shifted rearward (with the CG) with the vehicle height kept constant; i.e. the lower forebody inclination angle is decreasing, thus making the vehicle sharper. As the engine is shifted rearward, we observe specific monotonic trends and tradeoffs that result in the following PROS and CONS:

- PROS: trim AOA decreases, trim lift remains nearly constant;

- CONS: trim elevator increases (CG rearward), trim drag increases, trim L/D decreases, RHP pole increases.

We also observe the following more complex (non-monotonic) behavior:

- trim fuel rate decreases (min near $55 \mathrm{ft}$ ) and then increases;

- trim FER decreases (min near $45 \mathrm{ft}$ - thrust/acceleration margin increases) and then increases (thrust/acceleration margin decreases).

\begin{tabular}{|l|l||l|}
\hline Property & Pro & Con \\
\hline \hline Trim Lift & Almost Constant & Almost Constant \\
Trim Drag & & Increases monotonically \\
Trim L/D & & Decreases monotonically \\
Trim AOA & Decreases monotonically & \\
Trim Elevator & & Increases monotonically \\
Trim FER & Decreases till $45 \mathrm{ft}$ & Increases after $45 \mathrm{ft}$ \\
Trim Fuel Rate & Decreases till $55 \mathrm{ft}$ & Increases after $55 \mathrm{ft}$ \\
RHP Pole & & Increases monotonically \\
RHP Zero & Increases after $45 \mathrm{ft}$ & Decreases till $45 \mathrm{ft}$ \\
RHP Z/P Ratio & & Decreases monotonically \\
\hline
\end{tabular}

Table 5. Trends for Rearward Engine Shift

Lower Forebody Angle Increase. Table 6 summarizes trends for an increasing lower forebody angle. Here, the horizontal engine location is fixed and the engine is moved downward - thus increasing the height of the vehicle. As the lower forebody angle is increased, we observe specific monotonic trends and tradeoffs that result in the following PROS and CONS: 
- PROS: trim AOA decreases, RHP zero increases, RHP zero increases, RHP zero-pole ratio almost constant;

- CONS: trim elevator increases, trim drag increases, trim L/D decreases, trim FER increases, trim fuel rate increases, RHP pole increases.

Non-monotonic patterns are not observed in this case.

\begin{tabular}{|l|l||l|}
\hline Property & Pro & Con \\
\hline \hline Trim Lift & Almost Constant & Almost Constant \\
Trim Drag & & \\
Trim L/D & & Decreases monotonically \\
Trim AOA & Decreases monotonically & \\
Trim Elevator & & Increases monotonically \\
Trim FER & & Increases monotonically \\
Trim Fuel Rate & Decreases monotonically & \\
RHP Pole & & Increases monotonically \\
RHP Zero & Increases monotonically & \\
RHP Z/P Ratio & Almost constant & Almost constant \\
\hline
\end{tabular}

Table 6. Trends for Increasing Lower Forebody Inclination

Rearward CG Shift. Table 7 summarizes trends for a rearward shifting CG. As the CG is shifted rearward, we observe specific monotonic trends and tradeoffs that result in the following PROS and CONS:

- PROS: trim AOA decreases, trim fuel rate almost constant;

- CONS: trim elevator increases, trim drag increases, trim L/D decreases, trim FER increases, RHP pole increases, RHP zero decreases, RHP zero-pole ration decreases.

Non-monotonic patterns are not observed in this case.

\begin{tabular}{|l|l||l|}
\hline Property & Pro & Con \\
\hline \hline Trim Lift & Almost Constant & Almost Constant \\
Trim Drag & & Increases monotonically \\
Trim L/D & & Decreases monotonically \\
Trim AOA & Decreases monotonically & \\
Trim Elevator & & Increases monotonically \\
Trim FER & & Increases monotonically \\
Trim Fuel Rate & & Almost constant \\
RHP Pole & & Increases monotonically \\
RHP Zero & & Decreases monotonically \\
RHP Z/P Ratio & & Decreases monotonically \\
\hline
\end{tabular}

Table 7. Trends for Rearward CG Shift

Vehicle Mass Increase. Table 8 summarizes trends for increasing mass. As the vehicle mass is increased, we observe specific monotonic trends and tradeoffs that result in the following PROS and CONS:

- PROS: RHP pole decreases, RHP zero-pole ratio almost constant;

- CONS: trim elevator and trim AOA increase, trim FER and fuel rate increase, RHP zero decreases.

We also observe the following more complex (non-monotonic) behavior: 
- trim L/D is a concave down function of mass for a fixed Mach and altitude; mass at which peak occurs increases with Mach (altitude fixed); decreases with altitude (Mach fixed).

\begin{tabular}{|l|l||l|}
\hline Property & Pro & Con \\
\hline \hline Trim Lift & Increases monotonically & \\
Trim Drag & & Increases monotonically \\
Trim L/D (peak) & Increases with Mach (altitude fixed) & Decreases with altitude (Mach fixed) \\
Trim AOA & & Increases monotonically \\
Trim Elevator & & Increases monotonically \\
Trim FER & & Increases monotonically \\
Trim Fuel Rate & & Increases monotonically \\
RHP Pole & Decreases monotonically & \\
RHP Zero & & Decreases monotonically \\
RHP Z/P Ratio & Almost constant & Almost constant \\
\hline
\end{tabular}

Table 8. Trends for Increasing Mass

\section{Vehicle Optimization}

Various schemes have been considered for the optimization of space vehicles. Within ${ }^{66}$, a conceptual design process - that takes factors like cost analysis into consideration - is described. The non-hierarchical nature of the design process is illustrated and several optimization methods - parameter based, gradient based, and stochastic methods - are examined. Within ${ }^{67}$, a probabilistic approach to vehicle design is taken in order to account for uncertainties. For air-breathing hypersonic aircraft, the coupling between the airframe and the engine introduces additional constraints into the design process. The importance of a multidisciplinary design optimization approach is illustrated within ${ }^{68}$. A collaborative approach ${ }^{69}$ for launch vehicle design is considered within ${ }^{70}$. The design is decomposed into several subsystem design problems. A system level optimizer coordinates the integration of subsystem designs while taking into account intersubsystem coupling and constraints. Such a subsystem-based algorithm is well suited to exploit parallel computing architectures.

In this section, we address optimizing the generic carrot shaped hypersonic vehicle under consideration. The flat base assumption is made; i.e. $h_{e}=h_{i}, A_{n}=\frac{1}{A_{d}}$. The goal is twofold: (1) understand what configurations result when very simple optimizations are considered, (2) understand how the gap matric can be used to design a control-friendly vehicle.

Admissible Parameter Values. Optimizations are conducted over the following control surface and engine parameter values:

- Elevator area $S_{\text {elev }}$ :

$[8.5,25.5] \mathrm{ft}^{2}$ (nominal: $17 \mathrm{ft}^{2}$ )

- Horizontal elevator position $x_{e l e v}$ :

$[70,90] \mathrm{ft}$ from nose (nominal: $85 \mathrm{ft}$ )

- Inlet height $h_{i}$ :

[2.25,6.75] ft (nominal: $4.5 \mathrm{ft}$ )

- Lower forebody inclination $\tau_{1 L}$ :

$\left[4.2^{\circ}, 8.2^{\circ}\right]\left(\right.$ nominal: $\left.6.2^{\circ}\right)$

Overview of Trim Optimization Algorithm. A nonlinear simplex optimization method was used. No derivatives are calculated because trimming the vehicle results in each iteration being expensive. ${ }^{71,72}$ An overview of the vehicle optimization algorithm is as follows:

1. Choose cost function (e.g. trim AOA, trim fuel, trim elevator, trim FER, trim L/D);

2. Select values for parameters being optimized over; e.g. $S_{\text {elev }}, x_{\text {elev }}, h_{i}, \tau_{1 L}$;

3. Trim the vehicle at selected flight condition; i.e. Mach 8, $85 \mathrm{kft}$, level flight; 
4. Evaluate cost function using obtained trim values;

5. Repeat 2-4 until optimal configuration for chosen cost function is obtained.

ASU High performance Computing Cluster. All optimizations presented in this paper were performed using the Arizona State University (ASU) High Performance Computing (HPC) cluster. This cluster is part of ASU's Ira A. Fulton School of Engineering High Performance Computing Initiative. The HPC is composed of 220 dual quadcore Intel Xeon EM64T (Extended Memory 64 Technology) nodes - each with 16 gigabytes of RAM. The cluster also has a partition for running large numbers of serial jobs comprised of 185 nodes with dual Xeon MP 64bit processors.

Static Optimization Using ASU HPC: Computational Issues. While individual optimizations proceed in a serial fashion, the search space is partitioned into subspaces. Due to the nonlinear nature of the problem, a multistart algorithm was used (with at most $4^{4}=256$ initial conditions per optimization problem); i.e. several initial starting points are considered in each subspace. Optimization from an initial guess for a single operating point using the nonlinear (3DOF + flexibility) model takes approximately 15 minutes (on a $2.5 \mathrm{GHz}$ CPU with $1 \mathrm{~GB}$ of memory) to converge to a local minimum (for the static objectives). The results from all the initial conditions are examined and the optimum is chosen. On average, 256 initial guesses are chosen for each static objective minimization.

The $\bar{q}=2000$ psf fuel consumption objective optimization takes approximately an hour to converge to a local minimum (on a $2.5 \mathrm{GHz}$ CPU with $1 \mathrm{~GB}$ of memory) from an initial guess (in each iteration the plant must be trimmed at 7 points along the $\mathrm{q}_{2000}$ trajectory). Similar to the static optimization, a multistart algorithm was used (with at most $4^{4}=256$ initial conditions).

Trim Optimization Results (Mach 8, 85kft, Level Flight). Table 9 shows the results obtained by optimizing the following trim vehicle variables at Mach 8, 85kt (level flight): trim AOA, trim fuel, trim elevator deflection, trim FER, and trim L/D. Table 10 shows the parameters that result from the respective optimizations. Table 11 contains corresponding dynamical characteristics.

\begin{tabular}{|c|c|c|c|c|c|}
\hline Objective & $\begin{array}{l}\text { Trim } \\
\mathrm{L} / \mathrm{D}\end{array}$ & $\begin{array}{l}\text { Trim } \\
\text { FER }\end{array}$ & $\begin{array}{l}\text { Trim Fuel } \\
\text { Rate }\left(\frac{\text { slugs }}{\sec }\right) \\
\end{array}$ & $\begin{array}{c}\text { Trim } \\
\text { Elevator (deg) }\end{array}$ & $\begin{array}{c}\text { Trim } \\
\text { AOA (deg) }\end{array}$ \\
\hline (1) Maximize Trim L/D & 6.63 & 0.29 & 0.116 & 2.19 & 6.39 \\
\hline (2) Minimize Trim FER & 5.95 & 0.27 & 0.102 & 2.65 & 6.64 \\
\hline (3) Minimize Trim Elevator & 5.95 & 0.27 & 0.102 & 1.11 & 6.64 \\
\hline (4) Minimize Fuel Consumed (const. $\bar{q}$ ) & 5.89 & 0.27 & 0.102 & 3.11 & 6.64 \\
\hline (5) Minimize Trim Fuel Rate & 4.38 & 0.47 & 0.0361 & 3.93 & 2.19 \\
\hline (6) Nominal (with New Engine) & 4.30 & 0.35 & 0.0708 & 6.44 & 2.88 \\
\hline (7) Minimize Trim AOA & 3.28 & 0.49 & 0.0616 & 10.14 & 0.00271 \\
\hline
\end{tabular}

Table 9. Optimized Static Properties - Resulting Trim Values (Mach 8, 85 kft, Level Flight)

\begin{tabular}{|l||c|c|c|c|}
\hline Objective & $S_{\text {elev }}\left(\mathrm{ft}^{2}\right)$ & $x_{\text {elev }}(\mathrm{ft})$ & $h_{i}(\mathrm{ft})$ & $\tau_{1 L}(\mathrm{deg})$ \\
\hline \hline (1) Maximize Trim L/D & $\overline{25.5}$ & $\overline{90}$ & $\overline{6.75}$ & 5.24 \\
(2) Minimize Trim FER & 19.55 & $\overline{90}$ & $\overline{6.75}$ & $\underline{4.2}$ \\
(3) Minimize Trim Elevator & $\overline{25.5}$ & $\overline{90}$ & $\overline{6.75}$ & $\underline{4.2}$ \\
(4) Minimize Fuel Consumed (const. $\bar{q})$ & 18.19 & $\overline{90}$ & $\overline{6.75}$ & $\underline{4.2}$ \\
(5) Minimize Trim Fuel Rate & 16.15 & 81.76 & $\underline{2.25}$ & $\underline{4.2}$ \\
\hline (6) Nominal (with New Engine) & 17 & 85 & 4.5 & 6.2 \\
\hline (7) Minimize Trim AOA & 19.89 & 73 & 3.15 & $\overline{8.2}$ \\
\hline
\end{tabular}

Table 10. Optimized Static Properties - Resulting Vehicle Configurations/Parameters (Mach 8, 85 kft, Level Flight) 


\begin{tabular}{|l||c|c|c|}
\hline Objective & RHP Pole & RHP Zero & RHP Zero-Pole ratio \\
\hline \hline (1) Maximize Trim L/D & $1.60(-45.18 \%)$ & $5.83(-16.02 \%)$ & $3.6325(+53.18 \%)$ \\
(2) Minimize Trim FER & $1.95(-33.43 \%)$ & $5.62(-19.06 \%)$ & $2.8833(+21.59 \%)$ \\
(3) Minimize Trim Elevator & $1.48(-49.56 \%)$ & $5.80(-16.46 \%)$ & $3.928(+65.64 \%)$ \\
$(4)$ Minimize Fuel Consumed (const. $\bar{q})$ & $2.05(-30.07 \%)$ & $5.61(-19.15 \%)$ & $2.7416(15.61 \%)$ \\
(5) Minimize Trim Fuel Rate & $3.14(+7.23 \%)$ & $7.32(+5.44 \%)$ & $2.3319(-1.66 \%)$ \\
\hline (6) Nominal (with New Engine) & $2.93(0.00 \%)$ & $6.94(0.00 \%)$ & $2.37(0.00 \%)$ \\
\hline (7) Minimize Trim AOA & $3.55(+21.23 \%)$ & $6.34(-8.59 \%)$ & $1.788(-24.60 \%)$ \\
\hline
\end{tabular}

Table 11. Optimized Static Properties - Resulting Dynamic Characteristics (Mach 8, 85 kft, Level Flight)

Within Table 10, over bars imply that the maximum admissible parameter was achieved; underlines imply that the minimum admissible parameter bound was achieved. From Tables 9-10, one observes the following:

1. Maximizing Trim $L / D$. Maximizing trim L/D results in a small FER - marginally above that obtained when trim FER is directly minimized. As when trim FER is minimized, maximizing L/D also results in a large trim AOA. In short, maximizing trim L/D yields results comparable to minimizing trim FER. Maximizing trim L/D, however, results in a larger elevator surface area and lower forebody inclination vis-a-vis when trim FER is minimized. For this case, $S_{\text {elev }}, x_{\text {elev }}$, and $h_{i}$, were maximized to yield a maximally large elevator, a maximally effective elevator, a maximally large engine.

2. Minimizing Trim FER. Minimizing trim FER produces results that are comparable to those obtained when $\mathrm{L} / \mathrm{D}$ is maximized - with $\mathrm{L} / \mathrm{D}$ reduced $10.3 \%$ from the maximum L/D, FER reduced by $12.1 \%$, and AOA increased by only $3.9 \%$. In contrast to maximizing $\mathrm{L} / \mathrm{D}$, minimizing FER results in a much smaller elevator surface area $(23.3 \%)$ and lower forebody angle $(19.9 \%)$. For this case, $x_{e l e v}$, and $h_{i}$ were maximized while $\tau_{1 L}$ was minimized to yield a maximally effective elevator, a maximally large engine, and a very aerodynamic lower forebody.

3. Minimizing Trim Elevator. Minimizing trim elevator produces results (L/D, Fuel, FER, AOA) that are comparable to minimizing trim FER with less than half the elevator. For this case, $S_{\text {elev }}, x_{\text {elev }}$, and $h_{i}$ were maximized while $\tau_{1 L}$ was minimized to yield a maximally large elevator and effective elevator, a maximally large engine, and a very aerodynamic lower forebody.

4. Minimizing Fuel Consumed (const. $\bar{q}$ ). Minimizing the fuel consumed along a constant dynamic pressure trajectory produces results that are comparable to minimizing trim FER with $x_{\text {elev }}$ and $h_{i}$ maximized and $\tau_{1 L}$ minimized to yield a maximally effective elevator, a maximally large engine, and a very aerodynamic lower forebody. This optimization is more involved than the others being considered. Hence some additional explanation is required.

The objective here is to minimize the total fuel consumed while flying along the $\bar{q}=2000$ psf altitudeMach profile from Mach 5.52 at $70 \mathrm{kft}$ to Mach 11.08 at a 100kft. To do so, we approximate the total fuel consumed. For simplicity, vehicle mass changes are ignored. The method used to approximate fuel consumption is described below.

(a) Trim at Selected Points. Select points along the stated $\bar{q}=2000$ psf profile spaced $5 \mathrm{kft}$. This yields the

altitudes: $\quad[70,75,80,85,90,95,100] \mathrm{kft}$ and the corresponding

Machs: $\quad[5.52,6.21,6.98,7.85,8.81,9.88,11.08]$.

This divides the profile into 6 legs. Trim the vehicle at each point.

(b) Approximate Maximum Accelerations. Approximate the maximum horizontal acceleration possible at the left end point of each leg. Do so using the FER margin at each left end point. The FER margin is used to determine the maximum FER. This is used to compute the associated thrust $T h\left(F E R_{\max }\right)_{i}$. The trim $\operatorname{drag} D_{i}$ and trim AOA $\alpha_{i}$ are then used to approximate the maximum acceleration at the left end point: $a_{i} \approx\left[\frac{T h\left(F E R_{\max }\right)_{i} \cos \alpha_{i}-D_{i}}{m}\right]$ where $m$ is the total mass of the vehicle. 
(c) Determine Average Accelerations for Each Leg. Assume that the average acceleration in leg i is the mean of the two end-point accelerations; i.e. $\bar{a}_{i}=\frac{a_{i}+a_{i+1}}{2}$ where $a_{i}$ is the approximate maximum acceleration at the left end point of the $i^{\text {th }}$ leg and $a_{i+1}$ is that at the right end point.

(d) Approximate Time to Fly Each Leg. Approximate the time to fly leg i as $T_{i} \approx \frac{v_{i+1}-v_{i}}{\overline{a_{i}}}$.

(e) Approximate the average fuel rate for leg i $\left(\overline{\dot{F}_{i}}\right)$ as the mean of the fuel rates at the end points (where we have maximum acceleration); i.e. $\dot{\bar{F}}_{i}=\frac{\dot{F}_{i}+F_{i+1}}{2}$

(f) Approximate Fuel Consumed During Each Leg. Approximate the fuel consumed during leg i as $F_{i} \approx \overline{\dot{F}_{i}} \times T_{i}$

(g) Approximate Total Fuel Consumed Over Flight Profile. Approximate the total fuel consumed (objective to be minimized) as

$$
\text { Total fuel consumed } \approx \sum_{i=1}^{6} F_{i}
$$

(h) Inadmissible Designs. We also want to maintain a reasonable FPA. The average FPA for the $i^{\text {th }}$ leg is approximated as

$$
\bar{\gamma} \approx \frac{h_{i+1}-h_{i}}{v_{i} \times T_{i}}
$$

where $\left(v_{i}, h_{i}\right)$ are the velocity and altitude at the left end point of the $i^{\text {th }}$ leg and $h_{i+1}$ is the altitude at the right end point. Designs for which the average FPA in any leg exceeded $3^{\circ}$ were deemed inadmissible and discarded.

5. Minimizing Trim Fuel Rate. Minimizing trim fuel rate produces results that lie between those obtained when trim AOA is minimized and elevator is minimized (or trim FER is minimized or trim L/D is maximized). Surprisingly, for this case both $h_{i}$ and $\tau_{1 L}$ are minimized to yield a maximally small engine with a very aerodynamic lower forebody. This optimization results in low trim air mass flow to the engine (and low trim AOA) so that even with the resulting larger trim FER and increased trim thrust, the trim fuel rate is small.

6. Nominal Vehicle. Observe that the nominal values $S_{\text {elev }}=17, h_{i}=4.5, \tau_{1 L}=6.2$ are midpoints while the nominal value $x_{\text {elev }}=85$ is near the right end point. Note that this case can be viewed as a tradeoff between minimizing trim fuel rate and trim AOA when looking at trim L/D and elevator, elevator area, and lower forebody angle. Otherwise, trim FER, fuel rate, AOA, elevator rearward distance, and engine inlet height are larger.

7. Minimizing Trim AOA. Minimizing trim AOA uses excessive trim elevator for lift. This results in excessive drag and results in a small trim L/D. It also produces a large trim FER and small trim fuel rate. The resulting low trim fuel rate is due to the reduced trim air mass flow to the engine (due to the smaller trim AOA). From a vehicle configuration perspective, this case results in the largest lower forebody inclination (least aerodynamic shape) - achieving the maximum admissible value for $\tau_{1 L}$.

Motivation for Gap Metric Based Optimization. The gap metric represents a system-theoretic measure that quantifies the "distance" between two dynamical systems and whether or not a common controller can be deployed for the systems under consideration ${ }^{48}$. Within ${ }^{49}$, the gap between two LTI dynamical systems $\left(P_{1}, P_{2}\right)$ is defined as follows:

$$
g\left(P_{1}, P_{2}\right) \stackrel{\text { def }}{=} \max \left\{\inf _{Q \in H_{\infty}}\left\|\left[\begin{array}{l}
D_{1} \\
N_{1}
\end{array}\right]-\left[\begin{array}{l}
D_{2} \\
N_{2}
\end{array}\right] Q\right\|_{\infty}, \quad \inf _{Q \in H_{\infty}}\left\|\left[\begin{array}{l}
D_{2} \\
N_{2}
\end{array}\right]-\left[\begin{array}{l}
D_{1} \\
N_{1}
\end{array}\right] Q\right\|_{\infty}\right\}
$$

where $P_{1}=N_{1} D_{1}^{-1}, P_{2}=N_{2} D_{2}^{-1}$, and $\left(N_{i}, D_{i}\right)$ denotes a normalized right coprime factorization for $P_{i}$ $(i=1,2)$ in the sense of ${ }^{73}$. The gap metric (and the $\nu$ gap $^{74}$ ) has often been considered from a robustness perspective in the stabilization of feedback systems ${ }^{75}$. Within ${ }^{76}$, the authors relate the gap metric with traditional stability margins. The gap metric has also been considered for the design of controllers for space vehicles $^{77,78}$. Given the importance of this metric from a controller design perspective, it may be very useful 
to consider the gap metric during the vehicle design phase. This motivates the vehicle optimization which we consider below.

Vehicle Optimization Using Gap Metric: Minimizing Plant Variability. We now consider vehicle optimization using the gap metric. Details of the optimization are now given.

- The optimization is conducted with respect to the four parameters: $S_{\text {elev }}, x_{\text {elev }}, h_{i}, \tau_{1 L}$.

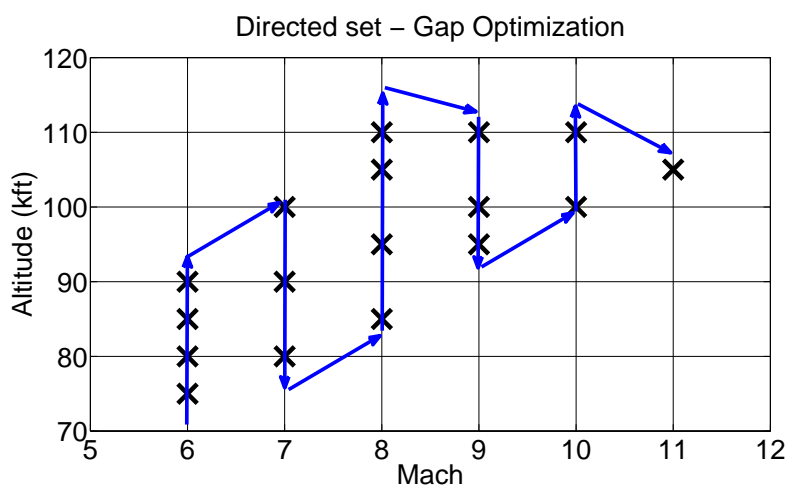

Figure 31. Directed Set of Level Flight Operating Points for Cumulative Gap Calculation

- Figure 31 shows the set of operating points used - a directed set - over which a cumulative gap is defined. The figure shows 17 operating points. (The operating points were selected in a way so as to span the desired trimmable region. How to do this systematically will be pursued in future work.) Associated with these 17 points are 16 gaps: $\operatorname{gap}_{i}=\operatorname{gap}\left(P_{i}, P_{i+1}\right), i=1,2, \ldots, 16$. The cumulative gap associated with these 17 points (along the directed set) is defined as follows:

$$
\text { cumulative gap } \stackrel{\text { def }}{=} \sum_{i=1}^{16} g a p_{i}
$$

A vehicle configuration is selected during optimization. The vehicle is trimmed at each point along the directed set. The gap between each pair of neighbors (along the directed set) is calculated and added to the running total for the vehicle configuration being considered.

- If a particular vehicle configuration is not able to trim at some point along the directed set, a penalty is added to the cumulative gap in lieu of the gap between this frozen point and its neighbors (along the directed set).

Gap Optimization Using ASU HPC: Computational Issues. Obtaining a gap-optimized vehicle from an initial guess takes approximately 4 hours on an ASU HPC 2.5GHz CPU with 1GB of memory. (During each iteration, the plant must be trimmed and linearized at each of the 17 selected operating points in altitude-Mach space.) As for the static optimization, a multistart algorithm was used (with at most $3^{4}=81$ initial conditions).

Vehicle Optimization Results: Gap-Optimized Versus Nominal. The parameters for the nominal vehicle (with new engine design) are as follows:

- Nominal Vehicle Parameters (with New Engine)

$$
S_{\text {elev }}=17 \mathrm{ft}^{2} \quad x_{\text {elev }}=85 \mathrm{ft} \quad h_{i}=4.5 \mathrm{ft} \quad \tau_{1 L}=6.2^{\circ}
$$

The cumulative gap for the nominal vehicle (with new engine design) is 4.4994 (mean gap $=0.28121$ ).

The final parameters for the gap-optimized vehicle are as follows:

- Gap-Optimized Vehicle Parameters

$$
S_{\text {elev }}=25.5 \mathrm{ft}^{2} \quad x_{\text {elev }}=90 \mathrm{ft} \quad h_{i}=6.12 \mathrm{ft} \quad \tau_{1 L}=4.2^{\circ}
$$

The cumulative gap for the optimized vehicle is 3.3989 (mean gap $=0.21243$ ) 
Note that with respect to the nominal vehicle, the gap-optimized vehicle has a

- larger elevator which is slightly shifted rearward (more effective elevator), larger engine inlet height (faster vehicle), smaller lower forebody inclination angle (more aerodynamic).

Modulo the simplicity of the nonlinear (3DOF + flexibility) model being used in this study and its associated limitations, the results given below suggest that these trends may significantly simplify control system design. More comprehensive studies are (of course) required in order to make definitive claims. Such a study could, for example, involve the 6 DOF models being developed within ${ }^{79}$.

RHP Pole Variation Over Level Flight Trimmable Region: Gap-Optimized Versus Nominal. Figure 32 (left) shows how the RHP pole varies across the trimmable region when the cumulative gap is minimized across the trimmable region (as discussed above). The variation for the nominal system (using the new engine design) is also given in Figure 32 (right).
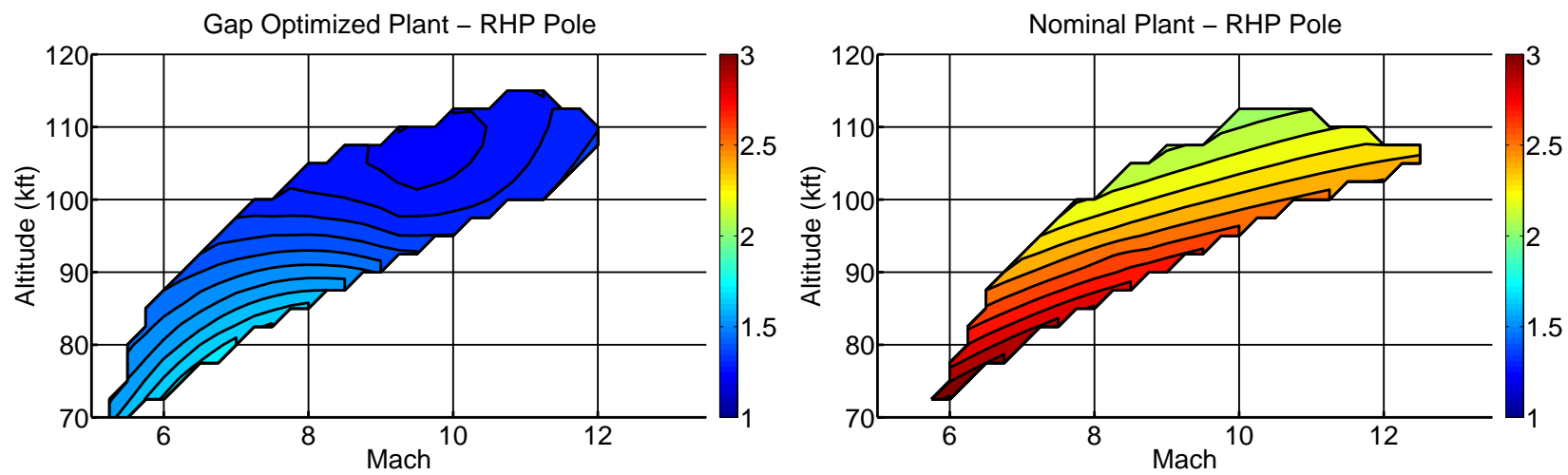

Figure 32. RHP Pole Over Trimmable Region - Nominal and Gap Optimized Vehicle

Figure 32 (left) shows that the

- RHP pole variation has decreased significantly when the vehicle is gap optimized; we will see that this implies that less adaptation (i.e. scheduling) will be required in the final control system design with respect to that required for the system associated with Figure 32 (right);

- Trimmable region has been expanded at low Mach and altitudes

\section{Control System Design Studies}

A classic decentralized inner-outer loop control system architecture was used to illustrate control design issues. Such an architecture was examined within [41]. It can be visualized as shown in Figure 33.

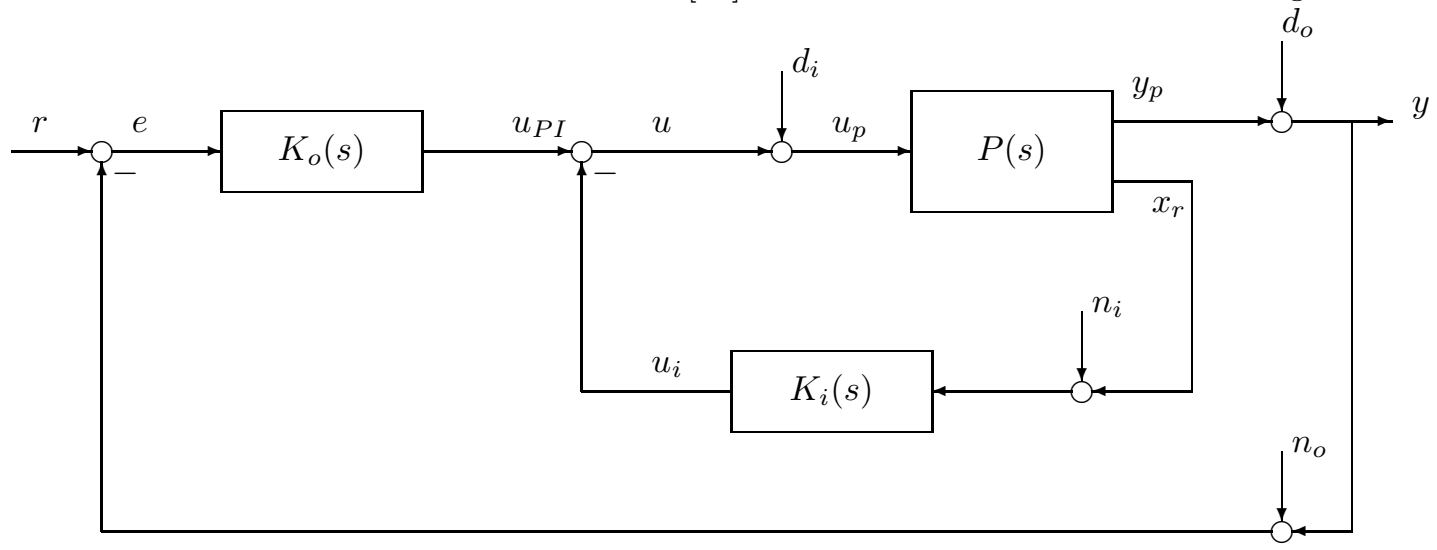

Figure 33. Inner Outer Loop Control System 

Outputs: $y_{p}=\left[\begin{array}{ll}v & \gamma\end{array}\right]^{T}$
Controls: $u=\left[\begin{array}{ll}F E R & \delta_{e}\end{array}\right]^{T}$
State Feedback: $x_{r}=\left[\begin{array}{ll}\theta & \dot{\theta}\end{array}\right]^{T}$

Overview of Nominal Control System Architecture. Within Figure 33, we have an inner loop controller:

$$
K_{i}(s)=\left[\begin{array}{cc}
0 & 0 \\
-g_{i} z_{i} \frac{a_{o}}{b_{o}}\left[\frac{s^{2}+b_{1} s+b_{o}}{s^{2}+a_{1} s+a_{o}}\right] & -g_{i} \frac{a_{o}}{b_{o}}\left[\frac{s^{2}+b_{1} s+b_{o}}{s^{2}+a_{1} s+a_{o}}\right]
\end{array}\right]
$$

and an outer loop controller:

$$
K_{o}(s)=\left[\begin{array}{cc}
\frac{g_{b}\left(s+z_{b}\right)}{s}\left[\frac{10}{s+10}\right]^{3} & 0 \\
0 & \frac{g_{\gamma}\left(s+z_{\gamma}\right)}{s}\left[\frac{20}{s+20}\right]^{3}
\end{array}\right]
$$

The inner-outer loop structure is now described.

1. Velocity Control Via Single Loop PI. A PI controller (with roll off) is used to control velocity. Two parameters are associated with velocity control: $\left(g_{b}, z_{b}\right)$. A simple anti-windup method (not discussed in the paper) is used to address the state-dependent thermal choking nonlinearity discussed earlier.

2. FPA Control Via Inner-Outer Loop. A PD (proportional plus derivative with notch) inner-loop controller is used on pitch to stabilize the vehicle's pitch dynamics and make the modified dynamics look friendly to the outer-loop FPA PI (proportional plus integral) controller. The inner loop's notch provides lead to assist with the first flexible mode's complex zero-pole pair (near $21 \mathrm{rad} / \mathrm{sec}$ ). As might be expected with this inner-outer loop structure, the inner loop is generally much faster than the outer loop. Its bandwidth must be large enough to stabilize the vehicle and follow outer-loop commands, but not too high so that the flexible dynamics are overly excited. (It should be noted that any practical "real-world" implementation of the PD controller should include additional roll off in order to attenuate high frequency noise $n_{i}$ resulting from $\theta$ and theta measurements.)

A PI (proportional plus integral with roll off) outer-loop controller is used for FPA. The outer loop is generally much slower than the inner loop. The maximum achievable outer loop bandwidth is limited by the vehicle's flexible dynamics as well as the right half plane zero associated with the elevator-FPA map.

Four parameters are associated with FPA control: $\left(g_{i}, z_{i}\right)$ for the inner-loop PD controller, $\left.g_{\gamma}, z_{\gamma}\right)$ for the outer-loop PI controller.

Command pre-filters are also included: $W_{b}=\frac{z_{b}}{s+z_{b}}$ for velocity reference commands, $W_{F P A}=\frac{z_{\gamma}}{s+z_{\gamma}}$ for FPA reference commands. (These reference command pre-filters are not shown within Figure 33.)

Additional information about controller parameter selection can be found within ${ }^{41}$. Nominal control system parameter values are given in Table 12 .

\begin{tabular}{|c|c|c|c|c|c|c|c|c|c|}
\hline$g_{i}$ & $z_{i}$ & $g_{\gamma}$ & $z_{\gamma}$ & $g_{b}$ & $z_{b}$ & $a_{0}$ & $a_{1}$ & $b_{0}$ & $b_{1}$ \\
\hline 1 & 4.5 & 21.23 & 0.404 & 2.2437 & 0.0278 & 30 & 900 & 15 & 250 \\
\hline
\end{tabular}

Table 12. Control System Parameters for Nominal Hypersonic Vehicle (Mach 8, 85kft, Level Flight)

\section{VI.A. Impact of Engine Location on Control System Design}

This section shows how a nominal control system design is impacted by the selection of the engine location with respect to the nose.

Impact on Velocity Loop's PI. As the engine is moved rearward (and the vehicle becomes more unstable), the (see Figure 34 - right)

- velocity loop's PI controller gain increases (smaller slope than FPA PI gain) 

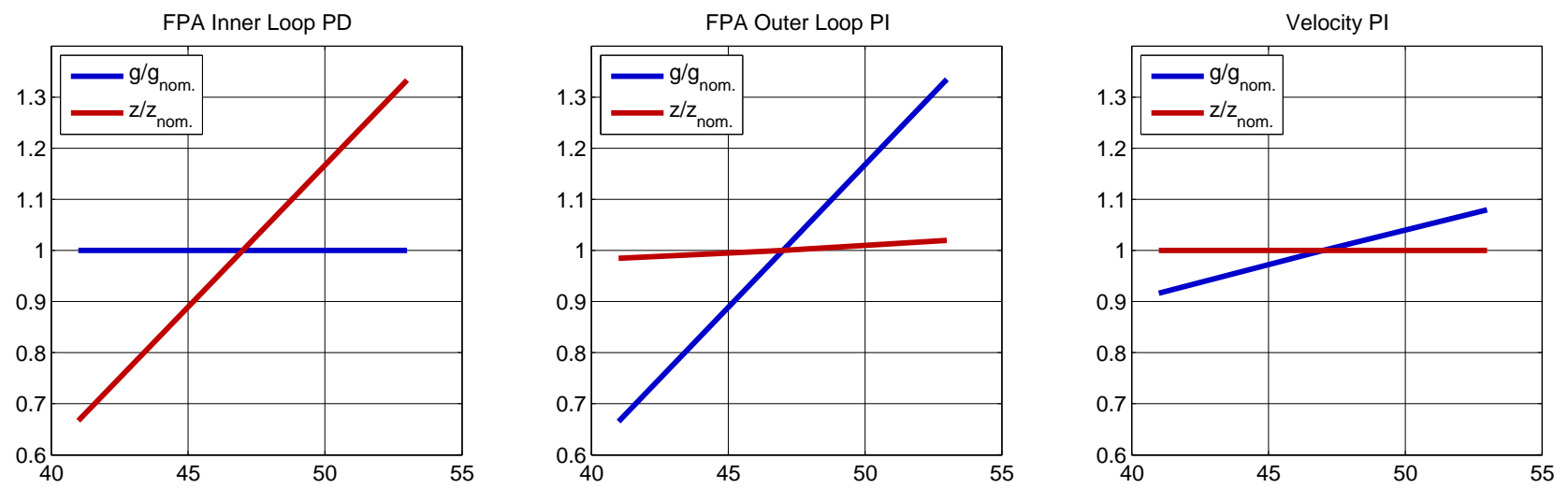

Figure 34. Control System Parameter Dependencies on Engine Location

- velocity loop's PI controller zero remains constant

Impact on FPA Inner-Loop PD. As the engine is moved rearward (and the vehicle becomes more unstable), the

- inner-loop PD gain remains constant

- inner-loop PD zero increases linearly - due to linearly increasing RHP Pole (see Figure 11)

Impact on FPA Outer-Loop PI. As the engine is moved rearward (and the vehicle becomes more unstable), the

- outer-loop PI controller gain increases; as inner-loop becomes more aggressive (due to increasing PD zero), the outer-loop must compensate - hence similar slope on outer-loop gain

- outer-loop PI controller zero increases linearly (with a small slope)

FPA Command Time Responses - Impact on Engine Location. Figure 35 shows the response to a step FPA command (with and without a command pre-filter) for three FPA control system designs each corresponding to a distinct engine location. The three designs were implemented to achieve a single target FPA time response; i.e. Figure 35 shows that a similar performance profile was achievable for the three distinct engine locations. Figure 36 contains the corresponding elevator responses. The figure shows that as the engine is moved rearward (and the vehicle becomes more unstable), more elevator is required. As the engine is moved rearward, so does the vehicle CG. This reduces the elevator's moment arm and it effectiveness - hence the need for more elevator as the engine is moved rearward.
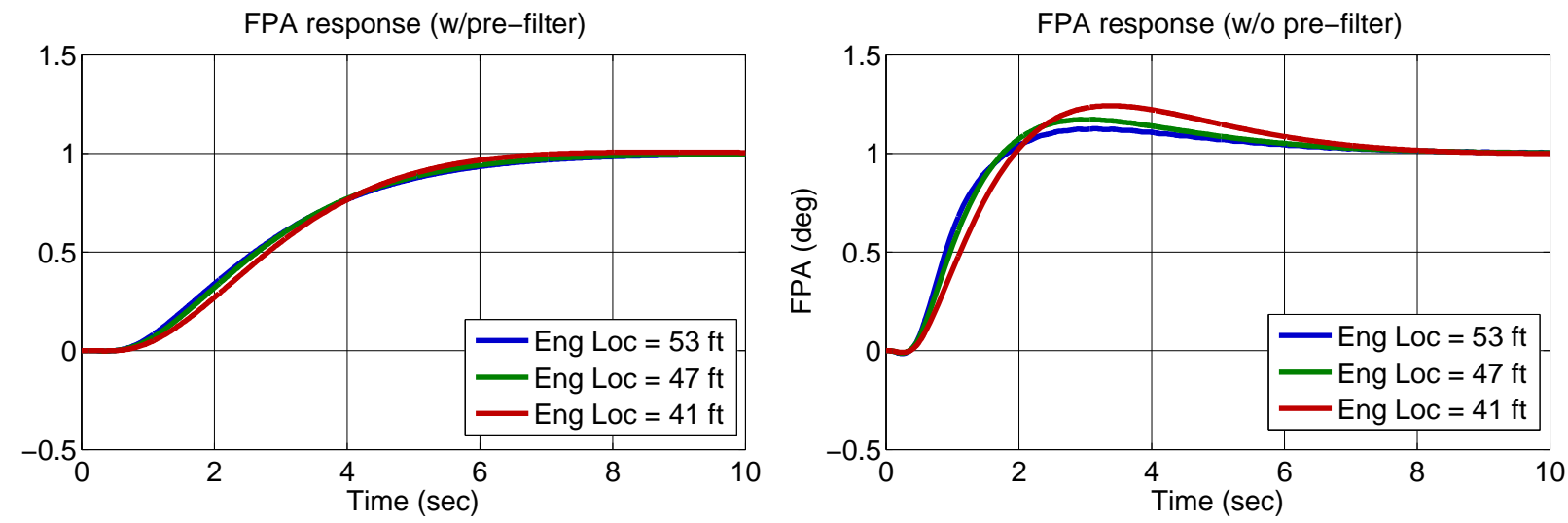

Figure 35. FPA Response to Step FPA Reference Commands: Control Designs for Three Engine Locations 
$\delta_{e}$ response (w/pre-filter)

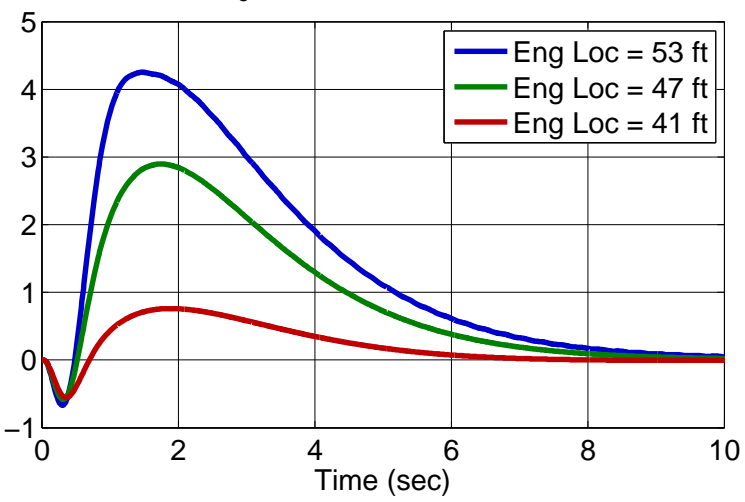

$\delta_{\mathrm{e}}$ response (w/o pre-filter)

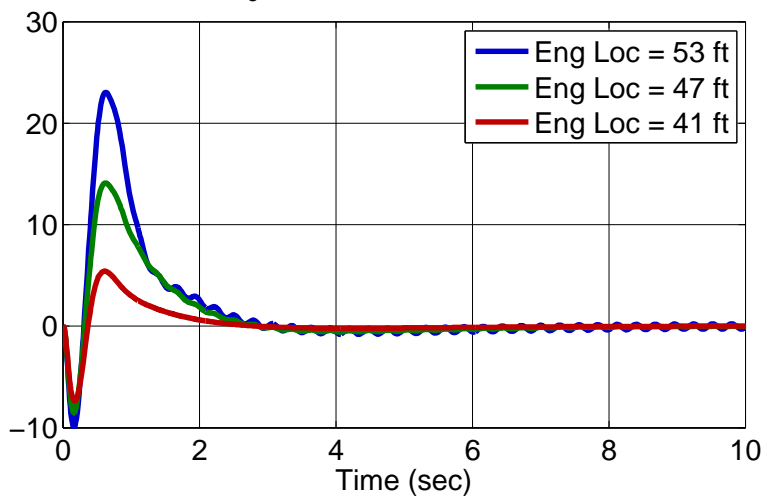

Figure 36. Elevator Response to Step FPA Reference Commands: Control Designs for Three Engine Locations

From the unfiltered elevator response in Figure 36 (right), one observes that the as the vehicle becomes more unstable the flexible mode effects become more pronounced; i.e. the control system cannot simultaneously provide the larger bandwidth required to stabilize the more unstable vehicle while not exciting the flexible dynamics. The following rough rules of thumb are observed:

- Minimum BW @ Controls: 2 times unstable pole

- Maximum BW @ Controls: 1/2-2/3 the frequency of the first flexible mode (21 rad/s)

The results show that a vehicle with a RHP pole between 5.5-7.5 becomes difficult to control with the chosen control system architecture; i.e. a vehicle that is too unstable may require some combination of the following:

- a more complex control system architecture (e.g. additional notches, MIMO)

- sensors capable of feeding back flexible mode information

- a more rigid vehicle; i.e. larger flexible mode frequencies

\section{VI.B. Nonlinear Simulations}

Within this section, we consider a constant $\bar{q}=2076$ psf flight profile followed by a pull-up maneuver.

The complete guidance and flight control systems may be visualized as shown below in Figure 37. To track constant dynamic pressure commands, a PI feedback structure (with roll off) was used for guidance:

$$
K_{\text {guidance }}(s)=\left[\frac{k(s+z)}{s}\right]\left[\frac{z_{\gamma}}{s+z_{\gamma}}\right]
$$

where $k=1 / 7846, z=15.7 . z_{\gamma}$ is determined from the outer-loop FPA controller - it is roughly selected to be the FPA outer-loop's PI controller's zero or larger. The second term in brackets is the FPA command pre-filter.

- Fuel depletion is modeled via linear decreasing mass from $6,154.1 \mathrm{lbs} / \mathrm{ft}$ to $3,049.0 \mathrm{lbs} / \mathrm{ft}$

- A simple PI guidance system (which processes dynamic pressure error $e_{\bar{q}}=\bar{q}_{2076 \text { psf }}-\bar{q}_{\text {actual }}$ ) is used to generates FPA guidance commands until Mach 8, 85 kft;

- A step velocity reference command is used to take the vehicle along $\bar{q}=2076$ psf from Mach 5.7 at 70 kft to Mach 8 at $85 \mathrm{kft}$.

- Once Mach 8, $85 \mathrm{kft}$ is reached, the pull-up maneuver is executed; To accomplish this, a FPA command of $3.5^{\circ}$ is held for 100 seconds.

Two vehicles are considered: 


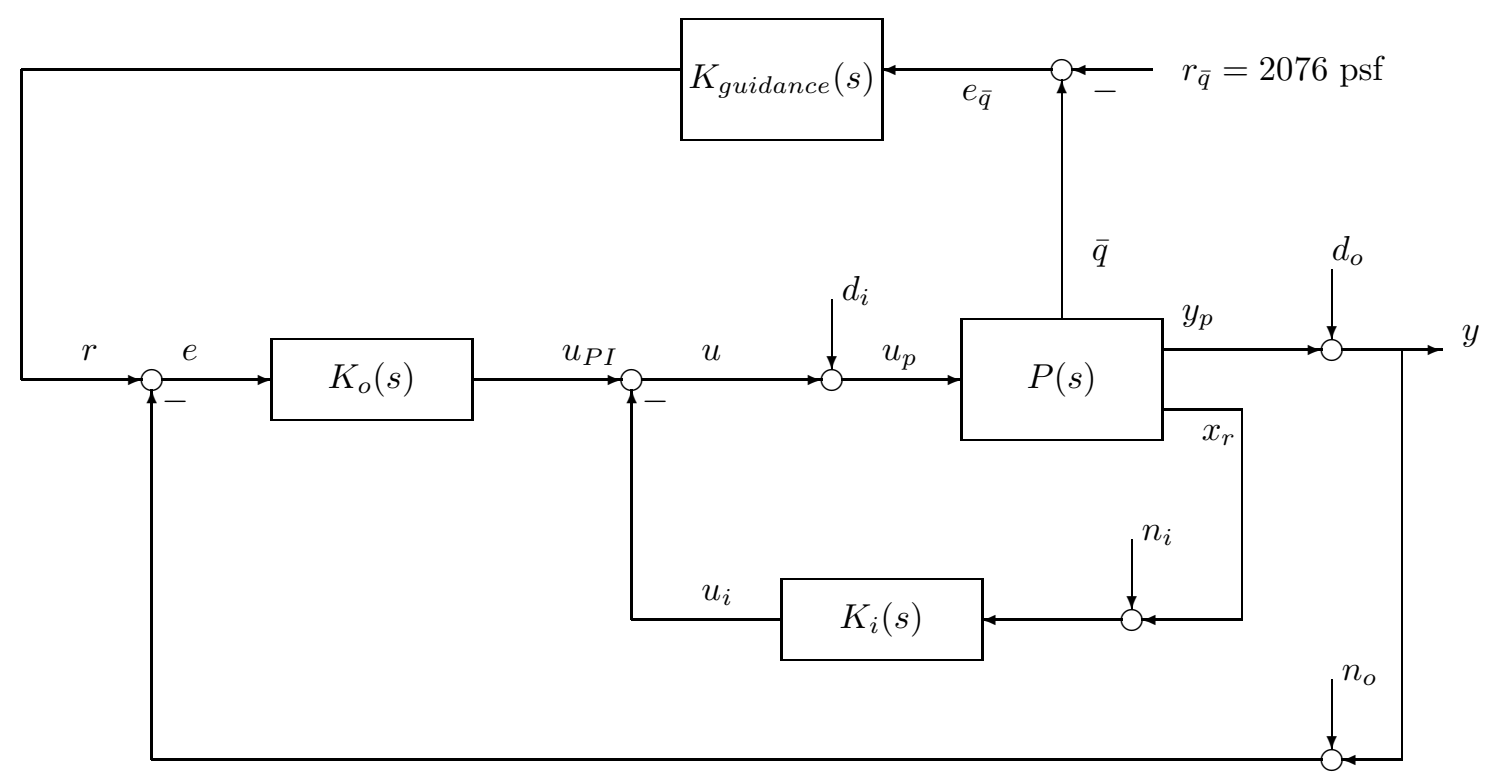

Figure 37. Inner-Outer Loop Architecture and Guidance System

1. One vehicle is the gap optimized vehicle (blue) - faster vehicle, larger engine, larger elevator, smaller lower forebody inclination (more aerodynamic);

2. The other vehicle is our nominal vehicle (with the new engine design) - slower vehicle, smaller engine, smaller elevator, larger lower forebody inclination (less aerodynamic);

Figure 38 contains relevant trajectory data. Figure 39 contains the corresponding Mach, FPA, AOA, and FER responses. Figure 40 contains the corresponding elevator responses as well as altitude-Mach profiles. 

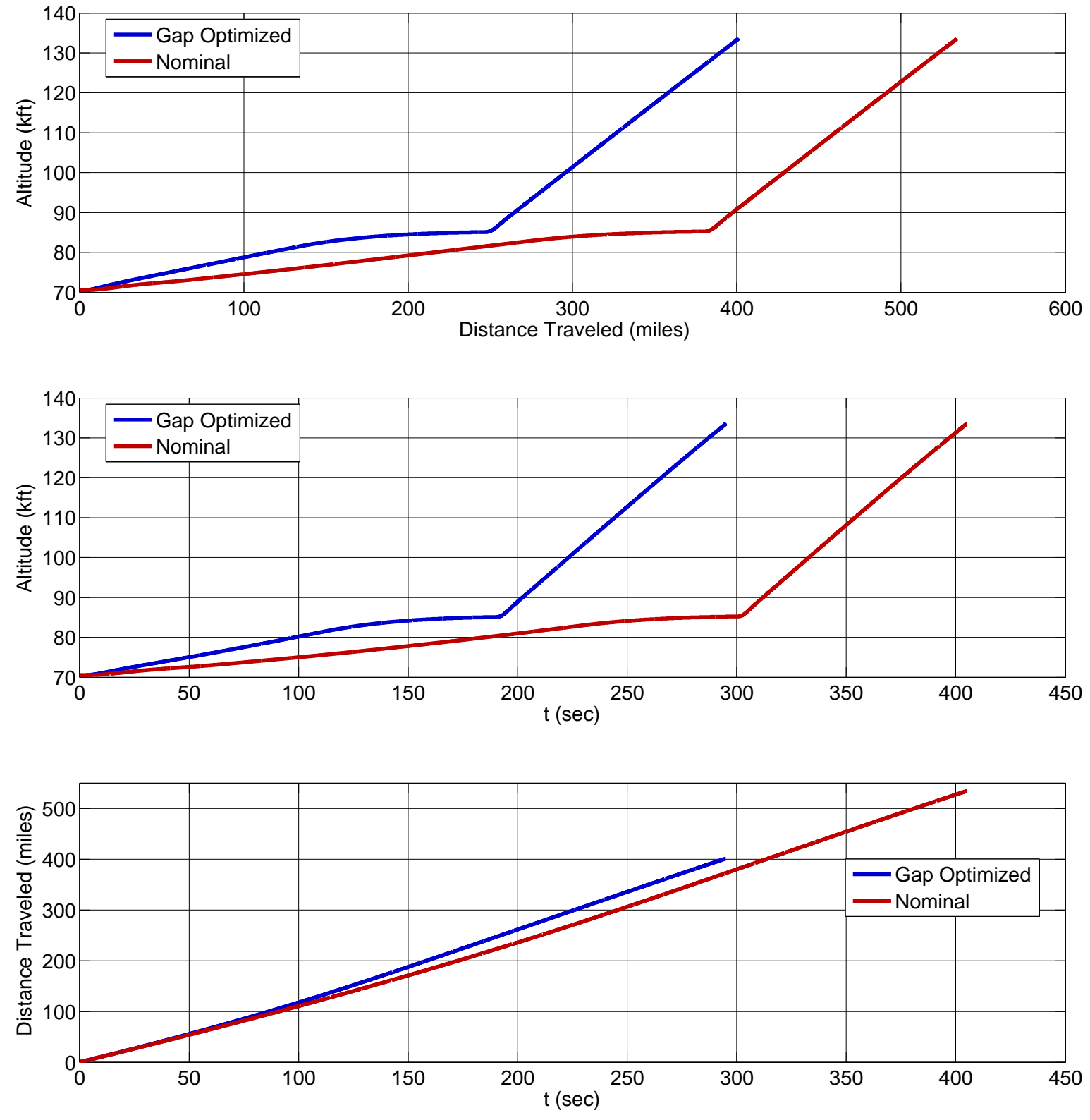

Figure 38. Nonlinear Time Responses for Vehicle Maneuver: Trajectory Data 

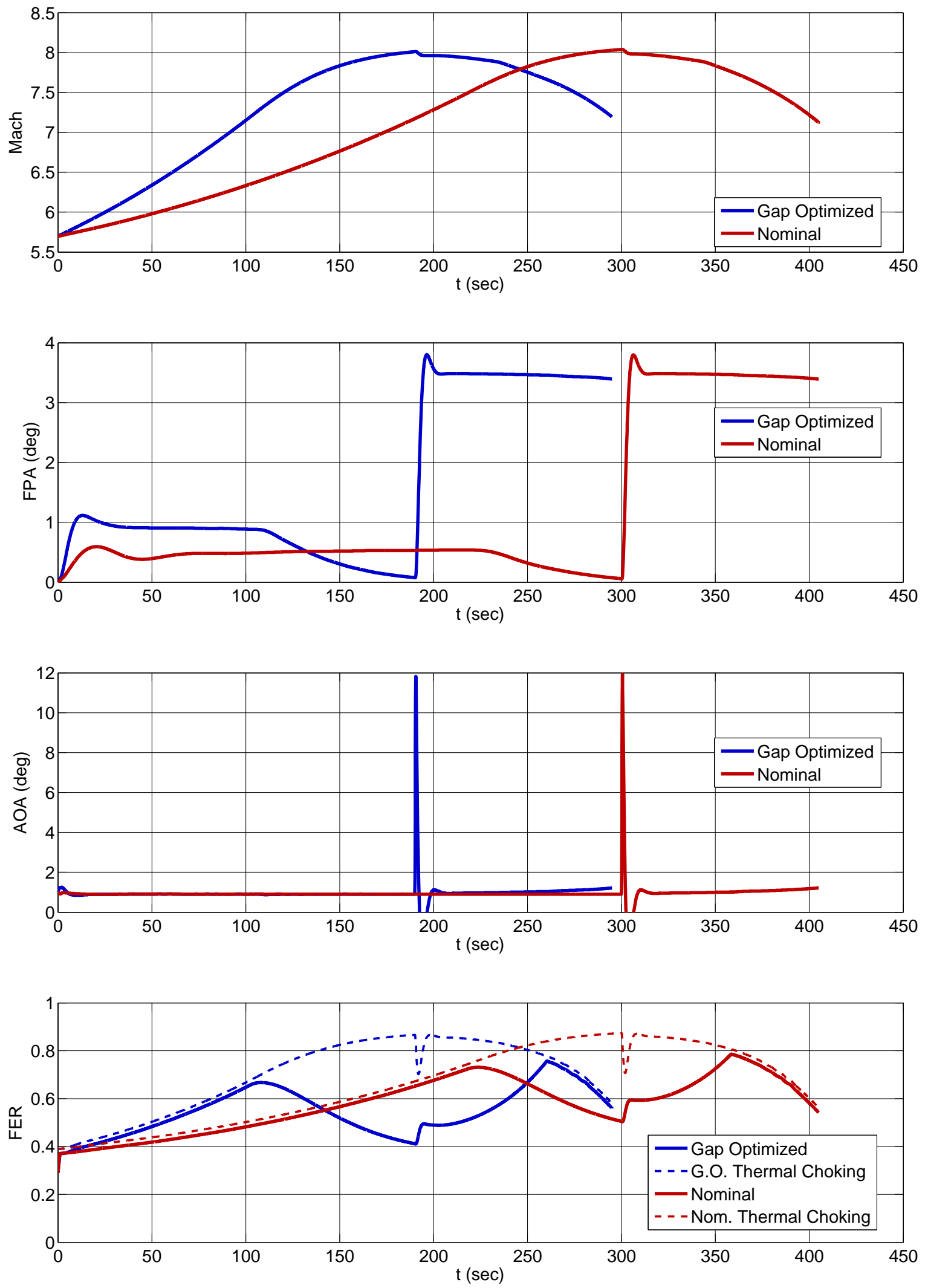

Figure 39. Nonlinear Time Responses for Vehicle Maneuver: Mach, FPA, AOA, FER 

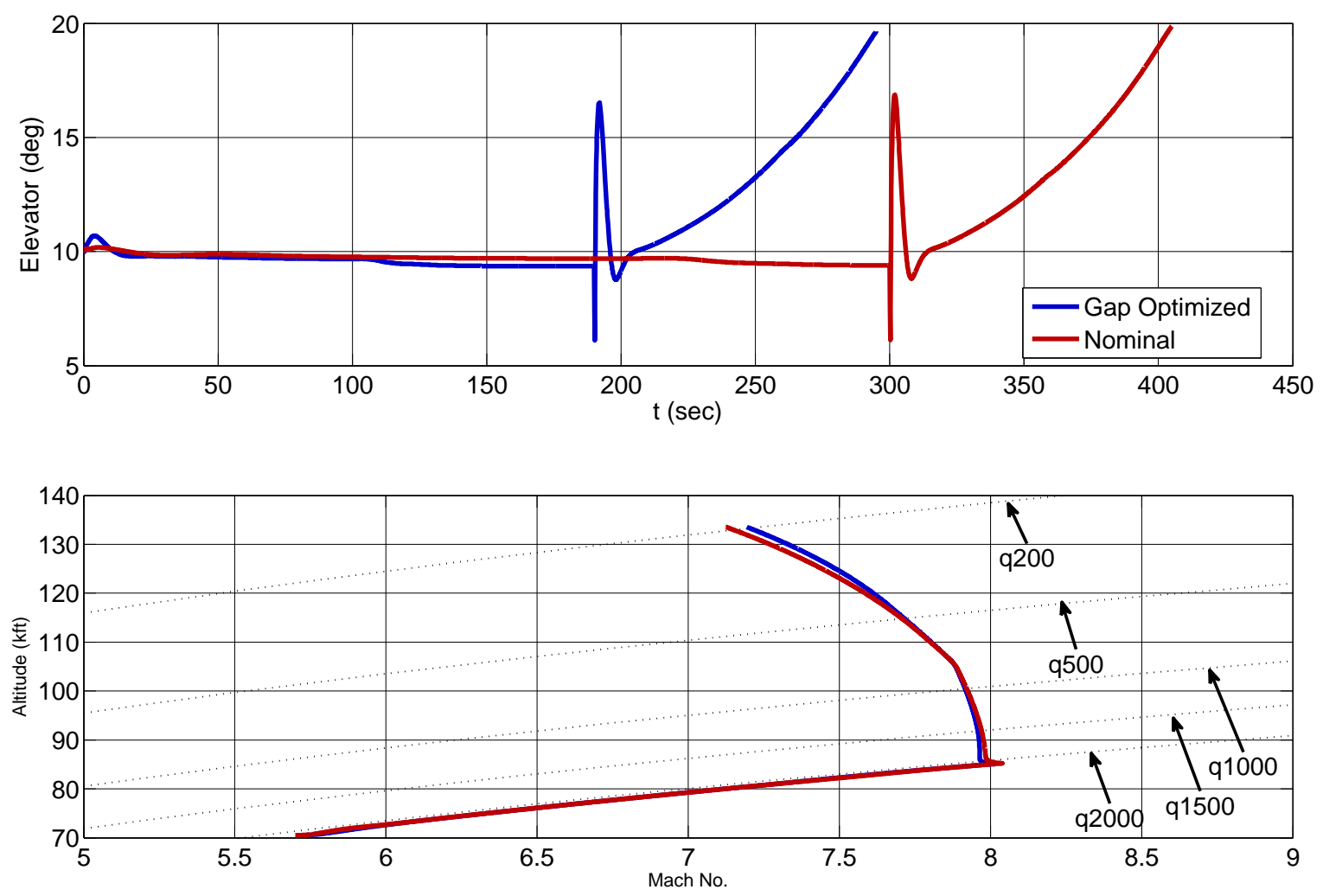

Figure 40. Nonlinear Time Responses for Vehicle Maneuver: Elevator, Altitude-Mach Profile

In short, the figures show that

- $\mathrm{AOA}<12^{\circ}$ with the peak occurring at the beginning of the pull-up

- The vehicle's climb to an altitude of $\sim 135 \mathrm{kft}$

- Dynamic pressure decreases to $\sim 200 \mathrm{psf}$

The benefits of the gap-optimized vehicle are as follows:

- The maneuver is completed $\sim 30 \%$ faster (due to increased thrust margin provided by the larger engine for the gap-optimized vehicle)

- While the non gap-optimized vehicle control system is scheduled on dynamic pressure to accomplish the pull-up maneuver, this is not required for the gap-optimized vehicle's control system. The gapoptimized vehicle thus requires a less sophisticated (less adaptive) control system.

For a more detailed discussion on gain scheduling of the nominal design, see [41]. Within [80] the author also examines gain scheduling issues for the nominal vehicle model.

\section{Summary and Directions for Future Work}

Summary. An engine redesign has been conducted based upon traditional as well as control-relevant metrics. A complete parametric study involving inlet capture area, diffuser area ratio, internal nozzle ratio, and nozzle exit area is presented to justify the engine redesign. Care was been taken to ensure that the engine parameters are feasible with respect to the geometry of the vehicle.

Vehicle geometry studies were also conducted. These involved engine location and forebody compression ramp angle. Their impact on the vehicle's trimmable envelope (region in altitude-Mach space), static (equilibrium) conditions at trim, and dynamic properties as they relate to control system design was addressed for 
level flight. These vehicle geometry trade studies were then used to obtain optimized vehicle parameters for various performance metrics at a single flight condition (Mach 8, $85 \mathrm{kft}$ ): (1) maximizing lift-to-drag (L/D), (2) minimizing fuel consumption, FER, elevator, or AOA. The gap metric was also used to minimize dynamic characteristics across the flight envelope (trimmable region). This was done with the intention of simplifying control system design. Finally, a simple (classic inner-outer loop decentralized) control system architecture was used to illustrate how vehicle parameter selection impacts control system design. Specifically, it was shown how a gap-optimized vehicle can simplify control system parameter scheduling. In short, the paper offers contributions to control-relevant vehicle design.

Conclusions. This paper has shown how a nonlinear (3DOF + flexibility) longitudinal model can be used for analysis, conducting vehicle trade studies, performing vehicle optimization, and control system design. It was shown that a gap-optimized vehicle can simplify the control system design/scheduling process.

Directions for Future Work. The work presented in this paper provides motivation for conducting comprehensive trade studies using higher fidelity vehicle models; i.e. $6 \mathrm{DOF}+$ flexibility $^{79}$. As such, the work motivates the development of general $6 \mathrm{DOF}$ tools that adequately address control-relevant modeling, analysis, and design issues for hypersonic vehicles during the early vehicle conceptualization/design phases. One specific concern will be to assess when conclusions obtained from a 3DOF model may be misleading. This work is currently being pursued by the team ${ }^{79}$.

\section{References}

${ }^{1}$ E. Baumann, C. Bahm, B. Strovers, R. Beck, and M. Richard, "The X-43A Six Degree of Freedom Monte Carlo Analysis," in $46^{\text {th }}$ AIAA Aerospace Sciences Meeting and Exhibit, Paper No. 2008-203, 2008.

${ }^{2}$ C. Peebles, Road to Mach 10: Lessons Learned from the X-43A Flight Research Program. AIAA, 2008.

${ }^{3}$ C. R. McClinton, "X-43 Scramjet Power Breaks the Hypersonic Barrier Dryden Lectureship in Research for 2006," $44^{\text {th }}$ AIAA Aerospace Sciences Meeting and Exhibit, Paper No. 2006-1, 2007.

${ }^{4}$ V. L. Rausch, C. R. McClinton, and J. L. Crawford, "Hyper-X Flight Validation of Hypersonic Airbreathing Technology," Technical Report, NASA Hyper-X Program Office, pp. 1 - 7, 1997.

${ }^{5}$ W. H. Heiser, D. T. Pratt, D. Daley, and U. Mehta, Hypersonic Airbreathing Propulsion. AIAA, 1994.

${ }^{6} \mathrm{M}$. Davis and J. White, "X-43A Flight-Test-Determined Aerodynamic Force and Moment Characteristics at Mach 7.0 ," J. of Spacecraft and Rockets, vol. 45, no. 3, pp. 472-484, 2008.

${ }^{7}$ M. A. Bolender and D. B. Doman, "A Non-Linear Longitudinal Dynamical Model of an Air-Breathing Hypersonic Vehicle," J. Spacecraft and Rockets, vol. 44 no. 2, pp. 373 - 387, 2007.

${ }^{8} \mathrm{M}$. Bolender, M. Oppenheimer, and D. Doman, "Effects of Unsteady and Viscous Aerodynamics on the Dynamics of a Flexible Air-Breathing Hypersonic Vehicle," in AIAA Atmospheric Flight Mechanics Conf, E6 Exhibit, Paper No. 2007-6397.

${ }^{9}$ M. Bolender and D. Doman, "Modeling Unsteady Heating Effects on the Structural Dynamics of a Hypersonic Vehicle," in AIAA Atmospheric Flight Mechanics Conf. and Exhibit, Paper No. 2006-6646, 2006.

${ }^{10} \mathrm{~J}$. Anderson, Hypersonic and High-Temperature Gas Dynamics. Second Edition. AIAA, 2006.

${ }^{11}$ E. A. Morelli, S. D. Derry, and M. S. Smith, "Aerodynamic Parameter Estimation for Flight 2 of the X-43A," Joint Army Navy NASA Air Force JANNAF Conf., 2005.

${ }^{12}$ S. D. Holland, W. C. Woods, and W. C. Engelund, "Hyper-X Research Vehicle Experimental Aerodynamics Test Program Overview," J. Spacecraft and Rockets, vol. 38, no. 6, pp. 828-835, 2001.

${ }^{13}$ Q. Wang and R. F. Stengel, "Robust Nonlinear Control of a Hypersonic Vehicle," J. Guidance, Control, and Dynamics, pp. $577-585,2000$

${ }^{14}$ B. Cobleigh, "Development of the X-33 Aerodynamic Uncertainty Model," NASA/TP-1998-206544, April 1998.

${ }^{15} \mathrm{H}$. Buschek and A. Calise, "Uncertainty Modeling and Fixed-Order Controller Design for a Hypersonic Vehicle Model," J. Guidance, Control and Dynamics, pp. $42-48,1997$.

${ }^{16}$ J. J. Bertin, Hypersonic Aerothermodynamics. AIAA Education Series, 1994.

${ }^{17}$ H. Buschek and A. J. Calise, "Robust Control of Hypersonic Vehicles Considering Propulsive and Aeroelastic Effects," Paper No. AIAA-1993-3762, pp. 1 - 11, 1993.

${ }^{18} \mathrm{~J}$. Young and J. Underwood, "Development of Aerodynamic Uncertainties for the Space Shuttle Orbiter ," J. of Spacecraft and Rockets, vol. 20, no. 6, pp. 513-517, 1983.

${ }^{19}$ J. T. Parker, A. Serrani, S. Yurkovich, M. A. Bolender, and D. B. Doman, "Control-oriented modeling of an air-breathing hypersonic vehicle," AIAA J. Guidance, Control, and Dynamics, 2007.

${ }^{20}$ M. Oppenheimer, D. Doman, M. Bolender, and T. Skujins, "A Flexible Hypersonic Vehicle Model Developed with Piston Theory," in AIAA Atmospheric Flight Mechanics Conference and Exhibit, Paper No. 2007-6396, 2007.

${ }^{21}$ D. Doman, M. Oppenheimer, and M. Bolender, "Progress in Guidance and Control Research for Space Access and Hypersonic Vehicles," Air Force Research Laboratory (AFRL), Wright Patterson Air Force Base, pp. 1 - 18, Tech. Rep., 2006.

${ }^{22}$ D. Sigthorsson, A. Serrani, S. Yurkovich, M. Bolender, and D. Doman, "Tracking Control for an Overactuated Hypersonic Air-Breathing Vehicle with Steady State Constraints," in AIAA Guidance, Navigation and Control Conf., Paper No. 2006-6558, 2006. 
${ }^{23}$ T. Adami, J. Zhu, M. Bolender, D. Doman, and M. Oppenheimer, "Flight Control of Hypersonic Scramjet Vehicles Using a Differential Algebraic Approach," in AIAA Guidance, Navigation and Control Conf., Paper No. 2006-6559, 2006.

${ }^{24}$ T. Williams, M. A. Bolender, D. Doman, and O. Mortaya, "An Aerothermal Flexible Mode Analysis of a Hypersonic Vehicle," Airforce Research Laboratory (AFRL), Wright Patterson Air Force Base, pp. 1 - 25,, Tech. Rep., 2006.

${ }^{25}$ J. T. Parker, A. Serrani, S. Yurkovich, M. Bolender, and D. Doman, "Approximate feedback linearization of an airbreathing hypersonic vehicle," Air Force Research Laboratory (AFRL), Wright Patterson Air Force Base, pp. 1 - 20, Tech. Rep., 2005.

${ }^{26}$ K. Groves, D. Sigthorsson, A. Serrani, S. Yurkovich, M. Bolender, and D. Doman, "Reference Command Tracking for a Linearized Model of an Air-Breathing Hypersonic Vehicle," in AIAA Guidance, Navigation and Control Conf. and Exhibit, Paper No. 2005-6144, 2005.

${ }^{27}$ K. P. Groves, A. Serrani, S. Yurkovich, M. Bolender, and D. Doman, "Anti-windup control for an air-breathing hypersonic vehicle model," Air Force Research Laboratory (AFRL), Wright Patterson Air Force Base, pp. 1 - 27, Tech. Rep., 2005.

${ }^{28}$ M. A. Bolender and D. B. Doman, "A Non-Linear Model for the Longitudinal Dynamics of a Hypersonic Air-Breathing Vehicle," in Proceedings of the 2005 Guidance, Navigation and Control Conf., Paper No. 2005-6255, 2005.

${ }^{29}$ A. Rodriguez, J. Dickeson, O. Cifdaloz, R. McCullen, J. Benavides, S. Sridharan, A. Kelkar, J. Vogel, and D. Soloway, "Modeling and Control of Scramjet-Powered Hypersonic Vehicles: Challenges, Trends, \& Tradeoffs," in AIAA Conf. on Guidance, Navigation and Control, AIAA-2008-6793, 2008.

${ }^{30}$ S. Torrez, J. Driscoll, M. Bolender, M. Oppenheimer, and D. Doman, "Effects of Improved Propulsion Modeling on the Flight Dynamic of Hypersonic Vehicles," in AIAA Atmospheric Flight Mechanics Conf. and Exhibit, Paper No. 2008-6386, 2008.

${ }^{31}$ S. Torrez, N. Scholten, J. Driscoll, M. Bolender, M. Oppenheimer, and D. D. Doman, "A Scramjet Engine Model Including Effects of Precomubstions Shocks and Dissociation," in $44^{\text {th }}$ AIAA/ASME/SAE/ASEE Joint Propulsion Conference and Exhibit, Paper No. 2008-6386, 2008.

${ }^{32}$ L. Fiorentini, A. Serrani, M. Bolender, and D. Doman, "Nonlinear Robust/Adaptive Controller Design for an Airbreathing Hypersonic Vehicle Model," in AIAA Guidnace Navigation and Control Conf. and Exhibit, Paper No. 2007-6329, 2007.

${ }^{33}$ G. Stein, "Respect the Unstable," IEEE Control Systems Magazine, August 2003, pp. 12 - 25.

${ }^{34} \mathrm{M}$. W. Oppenheimer and D. B. Doman, "Control of Unstable, Nonminimum Phase Hypersonic Vehicle," in IEEE Aerospace Conf., pp. $5782-5787,2006$.

${ }^{35}$ A. A. Rodriguez, Analysis and Design of Multivariable Feedback Control Systems. Control3D, LLC, 2004.

${ }^{36}$ M. Bolender and D. Doman, "Flight Path Angle Dynamics of Air-Breathing Hypersonic Vehicles," AIAA Guidance Navigation and Control Conf and Exhibit, Paper No. 2006-6692, Tech. Rep., 2006.

${ }^{37}$ P. Jankovsky, D. Sigthorsson, A. Serrani, S. Yurkovich, M. Bolender, and D. Doman, "Output Feedback Control and Sensor Placement for a Hypersonic Vehicle Model," in AIAA Guidance, Navigation and Control Conference and Exhibit, Paper No. AIAA 2007-6327, 2007.

${ }^{38}$ D. Sigthorsson, P. Jankovsky, A. Serrani, S. Yurkovich, M. Bolender, and D. Doman, "Robust Linear Output Feedback Control of an Airbreathign Hypersonic Vehicle," J. of Guidnace, Control and Dynamics, vol. 31, pp. 1-15, 2008.

${ }^{39}$ A. Culler, T. Williams, and M. Bolender, "Aerothermal modeling and dynamic analysis of a hypersonic vehicle," in AIAA Atmospheric Flight Mechanics Conference and Exhibit 2007 Paper. No. 2007-6395, 2007.

${ }^{40} \mathrm{~S}$. Torrez, J. Driscoll, D. Dalle, M. Bolender, and D. Doman, "Hypersonic vehicle thrust sensitivity to angle of attack and mach number," in AIAA Atmospheric Flight Mechanics Conference 2009, Paper No. 2009-6152, 2009.

${ }^{41}$ J. Dickeson, A. Rodriguez, S. Sridharan, J. Benavides, D. Soloway, A. Kelkar, and J. Vogel, "Decentralized control of an airbreathing scramjet-powered hypersonic vehicle," in AIAA Conf. on Guidance, Navigation and Control, AIAA-2009-6281, 2009.

${ }^{42} \mathrm{~S}$. Bhat and R. Lind, "Control-oriented analysis of thermal gradients for a hypersonic vehicle," in Proc. ACC '09. American Control Conference, 10-12 June 2009, pp. 2513-2518.

${ }^{43}$ K. Groves, A. Serrani, S. Yurkovich, M. Bolender, and D. Doman, "Anti-Windup Control for an Air-Breathing Hypersonic Vehicle Model," in AIAA Guidance, Navigation, and Control Conf. and Exhibit, Paper No. AIAA 2006-6557, 2006.

${ }^{44}$ A. Zinnecker, A. Serrani, M. Bolender, and D. Doman, "Combined reference governor and anti-windup design for constrained hypersonic vehicles models," in AIAA Guidance, Navigation, and Control Conference 2009, Paper No. 2009-6283, 2009.

${ }^{45}$ A. Teel and N. Kapoor, "The $\mathcal{L}_{2}$ anti-windup problem: Its definition and solution." in Proc. of the European Control Conf., 1997.

${ }^{46}$ D. Soloway, A. Rodriguez, J. Dickeson, O. Cifdaloz, J. Benavides, S. Sridharan, A. Kelkar, and J. Vogel, "Constraint Enforcement for Scramjet-Powered Hypersonic Vehicles with Significant Aero-Elastic-Propulsion Interactions," in 2009 American Control Conference, Accepted for Publication, 2009.

${ }^{47}$ J. D. Anderson, Modern Compressible Flow, $3^{\text {rd }}$ Edition. McGraw-Hill, 2002.

${ }^{48}$ K. Glover, G. Vinnicombe, and G. Papageorgiou, "Guaranteed multi-loop stability margins and the gap metric," in Proceedings of the 39th IEEE Conference on Decision and Control, 2000. 1988.

${ }^{49}$ T. T. Georgiou, "On the computation of the gap metric," in Proceedings of the 27th Conference on Decision and Control,

${ }^{50}$ B. M. Meyer, "Tip to Tail Preflight Prediction," in Joint Army Navy NASA Air Force (JANNAF) Conf., 2005.

${ }^{51}$ M. W. Oppenheimer and D. B. Doman, "A Hypersonic Vehicle Model Developed with Piston Theory," in AIAA 20066637,2006

${ }^{52}$ C. E. Cockrell, W. C. Englelund, R. D. Bittner, T. N. Jentink, A. D. Dilley, and A. Frendi, "Integrated Aeropropulsive Computational Fluid Dynamics Methodology for the Hyper-X Flight Experiment," J. of Spacecraft and Rockets, pp. 836 - 843, 2001. 
${ }^{53}$ C. Breitsamter, T. Cvrlje, B. Laschka, M. Heller, and G. Sachs, "Lateral-Directional Coupling and Unsteady Aerodynamic Effects of Hypersonic Vehicles," J. Spacecraft and Rockets, pp. 159 - 167, 2001.

${ }^{54}$ S. Berry, K. Daryabeigi, K. Wurster, and R. Bittner, "Boundary Layer Transition on X-43A," in $38^{\text {th }}$ Fluid Dynamics Conf. and Exhibit, Paper No. 2008-3736, 2008.

${ }^{55}$ S. A. Berry, A. H. Auslender, A. D. Diller, and J. F. Calleja, "Hypersonic Boundary-Layer Trip Development for HyperX," J. Spacecraft and Rockets, pp. 853 - 864, 2001.

${ }^{56}$ R. Starkey, D. Liu, R. Chang, and P. Chem, "Rapid Conceptual Design and Analysis of a Hypersonic Air-Breathing Missile ," in 15 th AIAA International Space Planes and Hypersonic Systems and Technologies Conf., 2008-2590, 2008.

${ }^{57}$ D. Glass, "Ceramic Matrix Composite (CMC) Thermal Protection Systems (TPS) and Hot Structures for Hypersonic Vehicles ," in $15^{\text {th }}$ AIAA International Space Planes and Hypersonic Systems and Technologies Conf/, 2008.

${ }^{58}$ D. H. Baldelli, R. Lind, and M. Brenner, "Nonlinear Aeroelastic/Aeroservoelastic Modeling by Block-Oriented Identification," J. Guidance, Control and Dynamics, pp. 1056 - 1064, 2005.

${ }^{59}$ R. Lind and M. Brenner, Robust Aeroservoelastic Stablity Analysis: Flight Test Applications. Springer, 1999.

${ }^{60}$ F. R. Chavez and D. K. Schmidt, "Analytical Aeropropulsive/Aeroelastic Hypersonic-Vehicle Model with Dynamic Analysis," J. Guidance, Control and Dynamics, pp. 1308 - 1319, 1994.

${ }^{61}$ D. K. Schmidt, "Dynamics and Control of Hypersonic Vehicles- The integration Challenge for the 1990's," in rd Intl. Aerospace Planes Conf., Paper No.1991-505\%, 1991.

${ }^{62}$ W. J. S. J. P. Weidner and J. A. Penland, "Scramjet Integration on Hypersonic Research Airplane Concepts," J. Aircraft, vol. 14, no. 5, pp. 460-466, May 1977.

${ }^{63}$ J. c. T. S. Srinivasan and K. J. Weilmuenster, "Simplified Curve Fits for the Thermodynamic Properties of Equilibrium Air, NASA Reference Publication 1181," NASA, Tech. Rep., 1987.

${ }^{64}$ R. Starkey and M. Lewis, "Critical design issues for airbreathing hypersonic waverider missles," J. of Spacecraft and Rockets, vol. 38, 2001.

${ }^{65}$ R. Nelson, Flight Stability and Automatic Control. McGraw-Hill Science/Engineering/Math, 1997.

${ }^{66}$ L. F. Rowell and R. D. Braun, "Multidisciplinary conceptual design optimization of space transportation systems," Journal of Aircraft, vol. Vol. 36, No. 1, pp. 218 - 226, 1999.

${ }^{67}$ N. D. Mavris, O. Bandte, and D. A. DeLaurentis, "Robust design simulation: A probabilistic approach to multidisciplinary design," Journal of Aircraft, vol. Vol. 36, No. 1, pp. 298 - 307, 1999.

${ }^{68}$ K. G. Bowcutt, "Multidisciplinary optimization of airbreathing hypersonic vehicles," Journal of Propulsion and Power, vol. Vol. 17, No. 6, pp. $1184-1190,2001$.

${ }^{69}$ R. Braun, "Collaborative optimization: An architecture for large-scale distributed design," Ph.D. dissertation, Stanford University, 1996 .

${ }^{70}$ R. D. Braun, A. A. Moore, and I. M. Kroo, "Collaborative approach to launch vehicle design," Journal of Spacecrafts and Rockets, vol. Vol. 34, No. 4, pp. 478 - 486, 1997.

${ }^{71}$ J. Nelder and R. Mean, "A Simplex Method for Funciton Minimization," Computer J., vol. 5, pp. 308-313, 1965.

${ }^{72}$ K. Bowcutt, "Hypersonic Aircraft Optimization Including Aerodynamic, Propulsion, and Trim Effects," no. AIAA-1992$5055,1992$.

${ }^{73} \mathrm{M}$. Vidyasagar, "The graph metric for unstable plants and robustness estimates for feedback stability," IEEE Transactions On Automatic Control, vol. Vol. 29, pp. 403-418, 1984.

${ }^{74}$ G. Vinnicombe, "Structured uncertainty and the graph topology," in Proceedings of the 30th Conference on Decision and Control, 1991.

${ }^{75}$ K. E. Ahmed, "The gap metric: Robustness of stabilization of feedback systems," IEEE Transactions On Automatic Control, vol. Vol. 30, No. 3, pp. 240 - 247, 1985.

${ }^{76}$ K. Glover, G. Vinnicombe, and G. Papageorgiou, "Guaranteed multi-loop stability margins and the gap metric," in Proceedings of the 39th IEEE Conference on Decision and Control, 2000.

${ }^{77}$ A. Fujimori, F. Terui, and P. N. Nikiforuk, "Flight control designs using v-gap metric and local multi-objective gainscheduling," in AIAA Guidance, Navigation, and Control Conference and Exhibit, AIAA 2003-5414, 2003.

78 _ , "Flight control design of an unmanned space vehicle using gain scheduling," Journal of Guidance, Control and Dynamics, vol. Vol. 28, No. 1, pp. 96 - 105, 2005.

${ }^{79}$ V. J. W. C. S. A. Kelkar, A. and A. Rodriguez, "Modeling and Analysis Framework for Early Stage Studies in ScramjetPowered Hypersonic Vehicles," in $16^{\text {th }}$ AIAA/DLR/DGLR International Space Planes and Hypersonic Systems and Technologies Conf., Paper No. 2009-7325, 2009.

${ }^{80}$ D. Sigthorsson, A. Serrani, M. Bolender, and D. Doman, "Lpv control design for over-actuated hypersonic vehicles models," in AIAA Guidance, Navigation, and Control Conference 2009, Paper No. 2009-6280, 2009. 